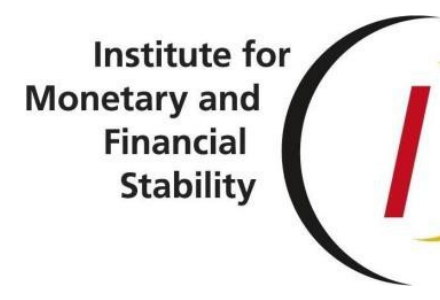

VOLKER WIELAND, ELENA AFANASYEVA, MEGUY KUETE, JINHYUK YOO

\title{
New Methods for Macro-Financial Model Comparison and Policy Analysis
}

Institute for Monetary and Financial Stability

GoETHE UNIVERSITY FRANKFURT AM MAIN 
This Working Paper is issued under the auspices of the Institute for Monetary and Financial Stability (IMFS). Any opinions expressed here are those of the author(s) and not those of the IMFS. Research disseminated by the IMFS may include views on policy, but the IMFS itself takes no institutional policy positions.

The Institute for Monetary and Financial Stability aims at raising public awareness of the importance of monetary and financial stability. Its main objective is the implementation of the "Project Monetary and Financial Stability" that is supported by the Foundation of Monetary and Financial Stability. The foundation was established on January 1, 2002 by federal law. Its endowment funds come from the sale of $1 \mathrm{DM}$ gold coins in 2001 that were issued at the occasion of the euro cash introduction in memory of the D-Mark.

The IMFS Working Papers often represent preliminary or incomplete work, circulated to encourage discussion and comment. Citation and use of such a paper should take account of its provisional character.

\section{Institute for Monetary and Financial Stability}

Goethe University Frankfurt

House of Finance

Theodor-W.-Adorno-Platz 3

D-60629 Frankfurt am Main

www.imfs-frankfurt.de I info@imfs-frankfurt.de 


\title{
New Methods for Macro-Financial Model Comparison and Policy Analysis*
}

\author{
Volker Wieland $^{\dagger, \S} \quad$ Elena Afanasyeva $^{\dagger} \quad$ Meguy Kuete $^{\dagger} \quad$ Jinhyuk Yoo $^{\dagger,+}$
}

August 23, 2016

\begin{abstract}
The global financial crisis and the ensuing criticism of macroeconomics have inspired researchers to explore new modeling approaches. There are many new models that deliver improved estimates of the transmission of macroeconomic policies and aim to better integrate the financial sector in business cycle analysis. Policy making institutions need to compare available models of policy transmission and evaluate the impact and interaction of policy instruments in order to design effective policy strategies. This paper reviews the literature on model comparison and presents a new approach for comparative analysis. Its computational implementation enables individual researchers to conduct systematic model comparisons and policy evaluations easily and at low cost. This approach also contributes to improving reproducibility of computational research in macroeconomic modeling. Several applications serve to illustrate the usefulness of model comparison and the new tools in the area of monetary and fiscal policy. They include an analysis of the impact of parameter shifts on the effects of fiscal policy, a comparison of monetary policy transmission across model generations and a cross-country comparison of the impact of changes in central bank rates in the United States and the euro area. Furthermore, the paper includes a large-scale comparison of the dynamics and policy implications of different macro-financial models. The models considered account for financial accelerator effects in investment financing, credit and house price booms and a role for bank capital. A final exercise illustrates how these models can be used to assess the benefits of leaning against credit growth in monetary policy.
\end{abstract}

JEL Classification: E17, E27, E32, E44, E52, E58

Keywords: model comparison, model uncertainty, monetary policy, fiscal policy, policy robustness, macro-financial models.

${ }^{*}$ This paper has benefited from helpful discussions with and comments by Thorsten Drautzburg, Simon Gilchrist, Johannes Pfeifer, John B. Taylor, Harald Uhlig, as well as participants at the Macroeconomics Conference in Chicago, the Macrofinancial Modeling Winter Meeting 2015 of the Becker Friedman Institute in New York, MACFINROBODS Annual Conference in Paris, the 10th Workshop on Macroeconomics and the Business Cycle at the Ifo Institute in Dresden, and AEA Meetings in San Francisco. Elena Afanasyeva gratefully acknowledges support from the FP7 Research and Innovation Funding Program (grant FP7-SSH2013-2). Jinhyuk Yoo and Meguy Kuete gratefully acknowledge support from the Research Center SAFE, funded by the State of Hessen initiative for research LOEWE.

${ }^{\S}$ Corresponding author. Contact: Institute for Monetary and Financial Stability, Goethe University Frankfurt. House of Finance, Theodor-Adorno-Platz 3, D-60629 Frankfurt, Germany. Tel.: +49-(0)6979833810,wieland@wiwi.uni-frankfurt.de.

Goethe University Frankfurt and IMFS

${ }^{\sharp}$ Bank of Korea. The views expressed in this paper are those of the authors and should not be attributed to the Bank of Korea. 


\section{Contents}

$\begin{array}{lll}1 & \text { Introduction } & 1\end{array}$

\begin{tabular}{|lll}
\hline 2 & Literature on model comparison and policy robustness & 2
\end{tabular}

2.1 How model comparison has been done so far . . . . . . . . . . . . 2

$2.2 \quad$ A recent example: Comparing effects of $2009-10$ fiscal stimulus . . . . . . . . 7

\begin{tabular}{|lll}
3 & A systematic approach to model comparison & 12
\end{tabular}

\begin{tabular}{|lll}
4 & Practical problems and a new platform & $\mathbf{1 6}$
\end{tabular}

4.1 Replication, computational implementation and model archiving . . . . . . . . . 16

\begin{tabular}{lll}
\hline 4.2 & User friendliness and a MATLAB-based platform for comparative analysis. . . . . 21
\end{tabular}

\begin{tabular}{|lll|}
\hline & Comparing fiscal and monetary policy transmission using the new platform & 27
\end{tabular}

5.1 Effects of fiscal stimulus: Sensitivity to structural parameters . . . . . . . . . . 27

5.2 Monetary policy transmission: Comparing generations of models . . . . . . . . . 31

5.3 Predicted effects of identified policy shocks: United States versus euro area . . . 37

6 Comparing implications of new macro-financial models $\quad 42$

6.1 Key characteristics: Investment finance, housing finance, banking capital. . . . . 42

6.2 Propagation mechanisms: Investment financing and the financial accelerator . . . 49

6.3 Propagation mechanisms: Housing finance and credit booms . . . . . . . . . . . 57

$6.4 \quad$ Propagation mechanisms: Financial intermediation and bank capital . . . . . . . 65

\begin{tabular}{|lll}
7 & How to assess policy robustness: An illustrative example & 68
\end{tabular}

\begin{tabular}{lll}
\hline & Critical assessment and outlook & 71
\end{tabular} 


\section{Introduction}

The global financial crisis and the ensuing criticism of macroeconomics have inspired researchers to develop new modeling approaches. There are many new models that aim to better integrate the financial sector in business cycle analysis. In these models, financial disturbances can have major macroeconomic consequences and the financial sector can amplify disturbances emanating from other sectors. They have potentially important implications concerning the effects of monetary and fiscal policy and the role of macroprudential and regulatory policy instruments. Thus, it is essential to be able to compare model implications for business cycle and policy analysis, and inform policy makers about policy strategies that are robust to model uncertainty. In fact, macroeconomic model comparison has a long tradition in the fields of monetary and fiscal policy. Central banks and international organizations have made much use of academic research on macroeconomic modelling, and they have invested staff resources in practical policy applications and sometimes large-scale comparison exercises.

In this paper, we report on new developments and new techniques for comparative analysis in macroeconomic modelling. It illustrates the usefulness of model comparison with practical applications concerning the effects of fiscal measures and the transmission of monetary policy. Furthermore, it gives an overview of recent macro-financial models and compares new propagation mechanisms that arise from different financial frictions.

The paper is meant to provide researchers, graduate students, economists at policy institutions, as well as business cycle analysts with access to a variety of macroeconomic models that reflect different theoretical paradigms, and to new hands-on tools for comparative analysis of policy and business cycle implications. A new on-line macroeconomic model archive together with a computational platform for model comparison takes center stage. It builds on and extends recent work on model comparison by Taylor and Wieland (2012), Wieland et al. (2012) and Schmidt and Wieland (2013). The computational platform for model comparison, the Macroeconomic Model Data Base (MMB, henceforth), enables individual researchers to conduct systematic model comparisons and policy evaluations easily and at low cost 1 . Furthermore, it is straightforward to include new models and compare their empirical and policy implications to a large number of established benchmark models from academia and policy-making institutions.

Thus, the paper and associated tools should help users to investigate questions such as: What are key features of well-known macroeconomic models? How do they influence the implications these models have for policy making and business cycle analysis? To what extent have these implications changed over time, as new models have been developed? How can I easily replicate models other researchers have developed, so as to apply them to new policy questions, or to extend them with new theoretical insights? How can I include my model in a comparison with other models in order to show what is new about it and where it improves upon earlier work? Which policy prescriptions would perform well across a range of models and economies?

\footnotetext{
${ }^{1}$ The model archive and software are available for download at www.macromodelbase.com.
} 
Next, we review some key contributions to the literature on model comparison and highlight a recent example concerning fiscal stimulus in the Great Recession. Section 3 briefly describes a formal approach for making sure that comparisons across different models focus on comparable objects. Section 4 deals with practical issues in conducting comparisons including reproducibility and user-friendliness. Illustrative applications and extensions of earlier comparisons are presented in section 5 The in-depth comparison of financial propagation mechanisms is discussed in section 6. Section 7 gives an example how to evaluate policy robustness and section 8 concludes.

\section{Literature on model comparison and policy robustness}

\subsection{How model comparison has been done so far}

Model comparison has a long tradition in the fields of monetary economics and macroeconomic modelling. Typically, comparisons were not undertaken by individual researchers or small teams. Rather, comparative studies brought together several teams of researchers multiple times to obtain results. In such a setting, each team usually just works with the model they have developed.

Interest in medium- to large-scale comparisons has invariably been supported by central banks and international organizations who have been building and using macro models for decades. Decision makers at central banks and finance ministries typically rely on their staff economists to inform them on likely macroeconomic consequences of various policy actions. Furthermore, they have a strong interest in forecasts conditional on different policy measures. Macroeconomic models are central to fulfilling this task. And, since central bankers are interested in many scenarios, and want to know about effects on different markets and sectors of the economy, the staff of their institutions is often pushed to build (or acquire from other institutions) a fairly large model of the economy, or to maintain a suite of models that are useful for different questions.

In the following we review some of the contributions to this literature, the questions that were investigated, the methodological problems that presented themselves, results obtained as well as some currently hot topics.

1980s to early 1990s: Standardizing experiments, comparing policy multipliers

Between 1984 and 1993 a number of large-scale comparisons were undertaken, several of them coordinated by the Brookings Institution in Washington, DC. Results were made available in form of books with chapters being contributed by many well-known researchers in the field. These include Bryant et al. (1988), Bryant et al. (1989), Klein (1991) and Bryant et al. (1993).

Bryant et al. (1988) aimed at improving the empirical understanding of cross-border macroeconomic and policy interactions. The study focused on the effects of monetary and fiscal policy and compared policy multipliers. To this end, participants developed and implemented a set of standardized exercises within 12 multi-country econometric models. These included models from the International Monetary Fund (IMF), the Organisation for Economic Cooperation and Development (OECD), the Federal Reserve and other policy institutions as well as models developed 
by leading academic macroeconomists. As emphasized by Hughes-Hallett (1989) this was a very impressive undertaking at the time. The objective was to show to what extent and where, the different models produce different and potentially conflicting policy implications.

A key focus was to implement common policy experiments that are comparable across models. To this end, common baseline paths of variables and common shocks were constructed across the models. Furthermore, methods were proposed to derive standardized comparison objects, such as estimates of policy multipliers. Interestingly, the study proposed computational procedures to recover estimates of the coefficients of policy variables in 'final-form' equations, to cast models to IS-LM relations and to summarize model performances in simple analytic constructs (slopes of IS-LM curves, inflation-output-tradeoffs, partial policy multipliers). The study helped identifying many challenges for standardized model comparisons and addressed some of them. It also revealed substantial differences in dynamic policy multipliers across models. Frankel and Rockett (1988) used the results from the Brookings model comparison project to explore how important uncertainty about the true model is for policy.

The follow-up study by Bryant et al. (1989) investigated the effects of changes in U.S. government spending and U.S. monetary policy. The majority of models participating in the exercises featured adaptive expectations. The authors computed averages and standard deviations of domestic and cross-border effects of U.S. policies across 20 global econometric models. Predicted effects from individual models, however, varied considerably. This study raised a question to which extent such model averages could be seen as guideline for robust policy design. Another lesson of this comparison was the need to understand and evaluate the effects of policies on more variables, e.g., by decomposing the effects on output into its components rather than concentrating on this variable alone.

Another round of model comparisons was documented by Klein (1991). The study focused on dynamic multipliers of fiscal spending increases, monetary expansion, and supply shocks. Similarly to earlier studies, the authors found significant variation in the behavior of models, resulting in very different policy multipliers. They aimed to put forth a common policy experiment and to apply common methods to understand the sources of variability in the multipliers. Yet, the study also made clear the difficulties in achieving comparability. The participating model teams admitted that "it required more than a year of repeated meetings to agree on a set of inputs to be used in all models and to be sure that each model operator made the appropriate arithmetic calculations" , (chapter 1, page 8).

Bryant et al. (1993) continued the Brookings comparison project that began in 1984. This study aimed at evaluating alternative regimes for the conduct of national monetary policies and understanding the stabilization properties of alternative operating regimes. Policy regimes were represented as simple policy rules. Taylor (1993a) credits the comparative exercise of Bryant et al. (1993) as the testing grounds for what would later become known as the Taylor rule. In contrast with previous comparative studies that focused on policy multipliers alone, Bryant et al. (1993) was the first large-scale project to compare stabilization properties of monetary policy 
regimes across models. The editors concluded: "A principal conclusion of the book is that some simplified regimes for monetary policy are markedly less promising than others for achieving the stabilization objectives customarily sought by policy makers. Most notably, for a wide variety of circumstances, neither money targeting nor exchange rate targeting performs as well as a regime that targets either nominal GNP or the sum of real GNP and the inflation rate".

Of course, all these comparison exercises were performed at a time when simulation techniques were much less developed than today. This made the comparisons such a challenging task. In particular, shocks and exogenous processes were very different across models, which made it difficult to disentangle the effects of different patterns of stochastic shocks from different model structure and different policy regimes. In many cases, it was not possible to use a common structure of stochastic disturbances, common transition paths and terminal conditions. Different techniques in stochastic simulation were used to perform policy experiments, which again affected the comparability of results. Furthermore, quarterly and annual models were not fully comparable. Annual models would identify monetary policy very differently by construction. Therefore, one of the lessons from this wave of comparative studies concerned the need for more standardization in methodology. Also, they highlighted the importance of expectations formation with models assuming either adaptive or rational expectations. Importantly, these comparison exercises typically concluded with an urgent call for improving empirical model validation and estimation techniques.

\section{Late 1990s and early 2000s: New models, policy rules and robustness}

Large-scale model comparison resumed with Taylor (1999). First, there was a new generation of New Keynesian models with a microfoundation built around a representative agent framework in which a household maximizes utility over time. Yet, these models were still fairly small such as the models of Rotemberg and Woodford (1997) and McCallum and Nelson (1999). They were being compared to models from the earlier generation of New Keynesian models that also featured nominal rigidities and rational expectations but a microeconomic foundation that consisted of separate decision rules for a household's consumption or a firm's investment and production problems, rather than a consistent representative agent framework. These included Fuhrer (1997), one model from Bank of England staff economists and four models developed by staff at the Federal Reserve Board (FRB). Also, there were some models with adaptive expectations such as Rudebusch and Svensson (1999) and Ball (1999).

In terms of modeling and numerical solution techniques there had been much progress since the earlier studies. As pointed out in the introduction of the volume, participating models had certain common features that made it easier to compute key statistics such as the variances of inflation and output under different monetary policy rules. For example, it was possible to derive linear systems determining the endogenous variables as functions of lags of themselves, the policy rate and exogenous shocks.

A central objective was to present econometric evidence on which type of monetary policy rule is likely to be both efficient and robust when used as a guideline for the conduct of monetary policy 
in the United States. The stabilization performance of selected interest rate rules was evaluated across 9 models. Exploiting the improvements in modeling solution techniques, Levin et al.(1999) were able to optimize over classes of policy rules using four different models, including the largescale FRB-US model that was heavily used to inform policy makers at the Fed. Taylor (1999) concluded that simple policy rules worked well, their performance was surprisingly close to that of fully optimal policies. Furthermore, simple rules turned out to be more robust than complex rules across a variety of models.

There was disagreement about whether the central bank should react to the exchange rate and whether policy should respond to the lagged interest rate (interest rate smoothing). Furthermore, there was disagreement whether the interest rate should respond solely to a measure of expected future inflation. Follow-up work by Levin et al. (2003) found that rules that respond to forecasts with a horizon of more than one year are less robust and more prone to generating equilibrium indeterminacy than rules that respond to current observations or near-term forecasts.

With the creation of the euro area many new models were built to inform policy makers at the European Central Bank (ECB) and other European and international institutions. A special issue of Economic Modelling was put together by Hughes-Hallett and Wallis (2004) to present and compare models for the euro area. It was preceded by conferences bringing together modelers from central banks, international institutions and academia to discuss estimates from different models. The paper by Wallis (2004) presents comparative results from four models, the ECB's Area-WideModel and three established multi-country model (IMF's MULTIMOD model, NIGEM from the National Institute of Economic and Social Research in London and the QUEST model from the European Commission). He found the principal source of differences across the four models to be the different degree of forward-looking behavior they incorporate in their treatment of consumption and investment decisions and the setting of wages and prices. Of course, at that point area-wide models had to be estimated on on pre-EMU macroeconomic data. Hence substantial uncertainty remained about the stability of established empirical regularities.

\section{Building a model archive to provide easy access to model comparison}

The last fifteen years have experienced a massive surge in macroeconomic model building. A new generation of medium-size New Keynesian Dynamic Stochastic General Equilibrium (DSGE) models emerged following the contribution by Christiano et al. (2005), which was first circulated as a working paper in 2001. These models extended the microfoundations of the representative agent framework with additional rigidities, adjustment costs and behavioral economics features such as habit formation. Smets and Wouters (2003) estimated a version of such a medium-size model for the euro area and helped popularize the use of Bayesian estimation methods. Widely used solution and estimation techniques were implemented in the DYNARE software package produced by Juillard (2001) (see also Adjemian et al. (2011)).

These advances in model building, model solution, model estimation and software implementations prepared the ground for more easy access to model comparison and the analysis of policy 
robustness by small teams of researchers. Taylor and Wieland (2012) extended the earlier model comparisons with U.S. models to the new medium-size DSGE models. They compare three such models built and estimated for the U.S. economy with the earlier-generation multi-country model of Taylor (1993b). Somewhat surprisingly, despite all the differences in structural assumptions and estimation techniques and data sample, all four models considered produce strikingly similar output responses to monetary policy shocks. Here, we extend this analysis further in Section 5.2

Kuester and Wieland (2010) and Orphanides and Wieland (2013) studied the robustness of simple monetary policy rules, the latter study using 11 new models estimated on euro area data. Orphanides and Wieland (2013) find that rules optimized for just one model are not robust, as they often result in substantially worse performance in other models. Yet, they show that a simple (not optimized) difference rule reacting to current inflation and output growth performs quite well across models.

Wieland et al. (2012) brought together models from these and earlier comparative studies to build an archive of macroeconomic models for easy simulation and comparison. This archive together with a new platform for performing standardized comparative exercises will be presented and used in subsequent sections of this chapter.

\section{Hot topics: Fiscal policy, macro-financial modeling and macroprudential policy}

Following the global financial crisis and Great Recession, there is high demand for new and improved models. Issues of great interest include the impact of fiscal stimulus and consolidation, the effects of unconventional monetary policy measures and the interaction of the real and financial sectors of the economy. Furthermore, there are new policy instruments to evaluate in banking regulation and macroprudential policy making. As a result, many new macro-financial models are being developed.

There have been several model comparison studies regarding fiscal policy, among them Cogan et al. (2010), Cwik and Wieland (2011), Cogan et al. (2013) and two large-scale comparisons of fiscal multipliers across models and countries by Coenen et al. (2012) and Kilponen et al. (2015). Section 2.2 reviews results from this debate concerning the likely effects of fiscal policy near zero interest rates.

Of course, policy makers and modelers alike have been pre-occupied with the role of the financial sector as a source of disruptions and as an amplifier of other economic disturbances for some time. There are many new modeling approaches. Thus, comparative research can generate useful insights. As a first step, Gerke et al. (2013) have considered 5 models of the European economies that are based on theoretical contributions from the pre-crisis period and are currently employed by central banks in the Eurosystem. They compare open-economy models featuring the financial accelerator mechanism as in Bernanke et al. (1999) and/or collateral constraints in the spirit of Iacoviello (2005). The focus of the study is on qualitative comparison of impulse responses of macroeconomic and financial variables to a range of common shocks (e.g., monetary policy shocks, net worth shocks, loan-to-value shocks). The study concludes that models display 
qualitatively similar features, reflecting a common understanding in macro-financial linkages preceding the financial crisis and the Great Recession. The authors, however, emphasize the need for a new generation of macro-financial models.

Guerrieri et al. (2015) is one of the first comparative studies of models that explicitly consider risks emanating from the banking system itself. Five groups of modelers from the Federal Reserve Board participated in the study. The authors compare macroeconomic spillovers from a (standardized) shortfall in bank capital across five DSGE models. The shortfall in bank capital is modeled as a gradually-decaying pure transfer from the banking sector to the household sector.

The models under consideration exhibit many differences. There are nominal and real models, models solved with linear and nonlinear techniques, models featuring complementary approaches on modeling of the financial intermediation. The financial shock is carefully standardized. Responses of macroeconomic and financial variables vary substantially. Noteworthy, the range of model-based outcomes is contained in the confidence bands of a bivariate VAR, suggesting a similar degree of uncertainty in response to the financial shocks in the models as in the VAR. The authors identify several sources of differences in responses across models. For instance, modeling of different sources of bank funding (for example, inside vs. outside equity) and interactions between alternative sectors, which can provide credit in the economy, are found to be particularly relevant for the results.

Section 6 provides an overview of different approaches for modeling macro-financial interactions. It also presents a range of examples and new findings from model comparisons. The model archive to be presented allows individual researchers to conduct such comparisons fairly themselves, and to include their own model so as to identify its contributions relative to more established benchmarks.

\subsection{A recent example: Comparing effects of 2009-10 fiscal stimulus}

The ongoing debate about the benefits and drawbacks of discretionary fiscal stimulus provides an excellent example of the need for model comparisons. The Great Recession of 2008 and 2009 has triggered substantial interest in assessing the likely impact of fiscal measures. In response to the financial market meltdown and the sharp contraction of real GDP, central banks in advanced economies have first slashed interest rates for central bank liquidity and then resorted to quantitative easing in order to further expand their balance sheets as policy rates remained near zero percent. At the same time, governments have launched large-scale fiscal stimulus packages.

In the United States, for example, the American Reconstruction and Reinvestment Act (ARRA) of February 2009 comprised US\$ 787 billion of additional government purchases, transfers and tax reductions. The lion's share was planned to be spent over a period of five years reaching a peak in 2010. The European Union initiated the European Economic Recovery Plan (EERP) and euro area members states launched fiscal stimulus packages on the scale of $€ 175$ billion to be spent in the years 2009 and 2010. Clearly, when deciding on such large programs, policy makers should be informed of the likely quantitative impact. 


\section{Determinants of Keynesian multiplier effects}

Advocates of fiscal stimulus refer to the Keynesian multiplier effect and emphasize that it would increase in strength with constant interest rates. The multiplier effect arises in the textbook IS-LM model due to the static nature of the Keynesian consumption function, which assumes a positive relationship between consumption and current household income. Additional government spending results in more aggregate demand, more production and more income, which in turn feeds additional household consumption and hence yet another increase in income and so on. This multiplication suggests that an increase in government spending would induce a greater than one-for-one increase in overall GDP.

However, there are several countervailing forces. An increase in government borrowing to finance spending puts upward pressure on interest rates and exchange rates, which tends to reduce domestic consumption and investment as well as foreign demand for domestic goods. Future tax increases needed to pay off the debt act to reduce current and future consumption of households that consider their life-time income. Thus, the increase in government demand tends to crowd out private sector demand. Yet, if central banks keep interest rates unchanged, there is less crowding out and more room for multiplication.

Whether GDP ultimately goes up and by how much is a quantitative question. Answering it requires an empirically estimated macroeconomic model, which accounts for key structural features of the economy that impact on the relative magnitudes of the multiplier and crowding-out effects. Furthermore, the particular timing and path of government spending and taxes, the reaction of monetary policy, and the expectations of households and firms regarding the paths of fiscal and monetary measures exert influence on the ultimate effects of fiscal stimulus.

\section{Controversy about model-based evaluations of ARRA and the zero bound}

Several studies employed macroeconomic model comparisons in order to provide policy makers with quantitative estimates of the likely impact of the above-mentioned stimulus measures. In January 2009, Christina Romer, then-Chair of the President's Council of Economic Advisers, and Jared Bernstein, Chief Economist of the Office of the Vice-President, estimated that a lasting increase in government purchases of 1 percent of GDP would lead to a rapid increase in GDP of 1.6 percent persisting for at least five years. This multiplier effect was obtained by averaging the effects in two macroeconomic models - a model from an unnamed private sector forecasting firm and a model from the staff of the Federal Reserve Board - while assuming constant interest rates for the full simulation period. On this basis, Romer and Bernstein (2009) anticipated that the ARRA would raise GDP by $3.6 \%$ by the fourth quarter of 2010 and employment by 3.5 million. Their report served as important quantitative policy advice for U.S. President Obama and the Members of U.S. Congress.

In a study first circulated in March 2009 Cogan et al. (2010) questioned the validity of the Romer-Bernstein estimate and reported much smaller GDP effects for simulations with the multi- 
country model of Taylor (1993b) and the model of the U.S. economy by Smets and Wouters (2007). The Smets-Wouters model is representative of current thinking in macroeconomics. It is largely based on another empirically-estimated and widely-known medium-size New Keynesian model developed by Christiano et al. (2005). On this basis, Cogan et al. (2010) conclude that the likely impact of the ARRA and U.S. GDP would only be around 1/6 of the Romer-Bernstein estimate.

Crowding-out effects are more important in these models because they take into account the forward-looking behavior of households and firms. Regarding fiscal policy, the path for government purchases is anticipated based on the information published with the ARRA. Regarding monetary policy, the simulations assume that the interest peg lasts between one or two years, which is more consistent with market expectations at the time than the Romer-Bernstein assumption. Afterwards, the policy rate responds again to economic conditions as in the simple policy rule of Taylor (1993a). The period of constant interest rates is motivated by the lower-bound on interest rates. Due to availability of cash, a zero interest rate asset, savers need not accept negative rates on bank deposits. Thus, in a situation where the central bank reaction function calls for a negative policy rate, the rate would be constrained near zero. As a result, fiscal stimulus that raises GDP would not be followed right away with tighter monetary policy as in normal times.

Cogan et al. (2010) account for the negative effect of increased future (lump-sum) taxes on household income and current consumption, but not for the negative effect of distortionary taxation on potential growth (see Drautzburg and Uhlig (2015)). Furthermore, they extend the SmetsWouters model by including "rule-of-thumb" households. Such households consume their current income as prescribed by the Keynesian consumption function. The empirically-estimated share of Keynesian consumers is about $27 \%$. The presence of rule-of-thumb consumers and the anticipation that interest rates remain constrained at zero for two years raise the GDP impact to about $1 / 4$ of the Romer-Bernstein estimate.

In contrast to these findings, Christiano et al. (2011) suggest that under certain conditions Keynesian multiplier effects can be much larger than one, even in modern New Keynesian models, when the zero-bound constraint on monetary policy rates binds. They present simulations of particular shocks in a small New Keynesian model and in a version of the medium-size model of Christiano et al. (2005). Thus, there appears to be stark disagreement between Cogan et al. (2010) and Christiano et al. (2011) even though both studies rely on fairly similar modern New Keynesian macroeconomic models estimated to U.S. macro data and both try to account for implications of the zero lower bound.

\section{A large-scale comparison study}

To illustrate how additional model comparisons can help improve policy advice in light of such disagreements, it is instructive to take a peak at a large comparison exercise that was organized by the IMF. This exercise involved several teams of researchers from central banks and international institutions which met at an IMF conference to compare a set of standardized simulations of fiscal stimulus that each team implemented in its institutions' model. Key findings were summarized 
in the journal article by Coenen et al. (2012). The article involves 17 authors and 9 different macroeconomic models. The authors are staff members of 6 different institutions, the International Monetary Fund, the Federal Reserve Board, the Bank of Canada, the European Central Bank, the Organisation for Economic Cooperation and Development, and the European Commission. Seven models were developed and used by staff members from these institutions, while the other two models are from Cogan et al. (2010) and Christiano et al. (2011) (see Table 1).

Table 1: MOdels PARTICIPATING IN THE COMPARISON OF COENEN ET AL. (2012)

\begin{tabular}{|c|c|}
\hline Notation & Description \\
\hline $\mathrm{CEE}$ & Christiano et al. (2011) model \\
\hline CCTW & $\begin{array}{l}\text { Cogan et al. (2010) extension of Smets and Wouters } 2007) \\
\text { model with rule-of-thumb households }\end{array}$ \\
\hline IMF-GIMF & the IMF's Global Integrated Monetary and Fiscal Policy model \\
\hline FRB-US & the Federal Reserve Board's US model \\
\hline SIGMA & the Federal Reserve Board's two-country DSGE model \\
\hline Boc-GEM & the Bank of Canada's Global Economy Model \\
\hline EC-QUEST & the European Commissions QUEST model \\
\hline ECB-NAWM & the European Central Bank's New Area-Wide Model \\
\hline OECD & the OECD's macroeconomic model \\
\hline
\end{tabular}

Here, we review just one particular set of simulations from Coenen et al. (2012). In this comparison, all participating models are simulated with the same fiscal stimulus. The stimulus corresponds to the increase in government purchases as planned according to the ARRA and previously studied Cogan et al. (2010). Technically, the spending path is simulated as an anticipated sequence of discretionary shocks. Regarding monetary policy, three different scenarios are considered that differ according to the importance of the zero bound.

The findings are presented in Figure 1. This figure is identical to Figure 7 of Coenen et al. (2012). The bars shown in each panel are identical and show the time profile for government purchases planned under the ARRA. The simulations assume that market participants anticipate the execution of the announced purchases over the coming years according to this plan. The different lines shown in the panels indicate the estimated impact of these government purchases on GDP in different macroeconomic models. Models estimated with the euro area data are reported in the right column of panels, labeled "Europe", whereas the left column displays results for the models of the U.S. economy.

Regarding monetary policy, three different scenarios are considered. The first row of panels in Figure 1 displays results for the case of no monetary accommodation, i.e. nominal interest rates in each model are set according to a model-specific interest rate rule. Thus, interest rates rise along with the increase in GDP and inflation and dampen the stimulative effects of government spending. In this scenario, all models under consideration deliver an increase in GDP over the first 2.5 years of the stimulus. However, the increase in GDP remains well below the associated increase in government spending, as private demand is being crowded out by government demand. Some of 
Figure 1: ESTIMATED GDP EFFECTS OF PLANED ARRA SPENDING

United States

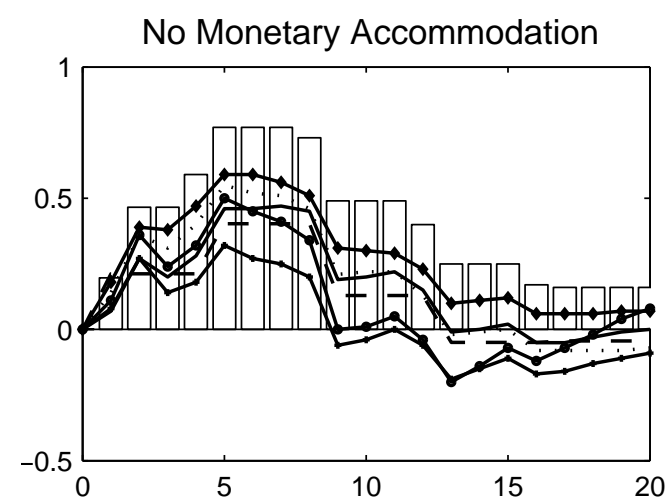

One Year of Monetary Accommodation

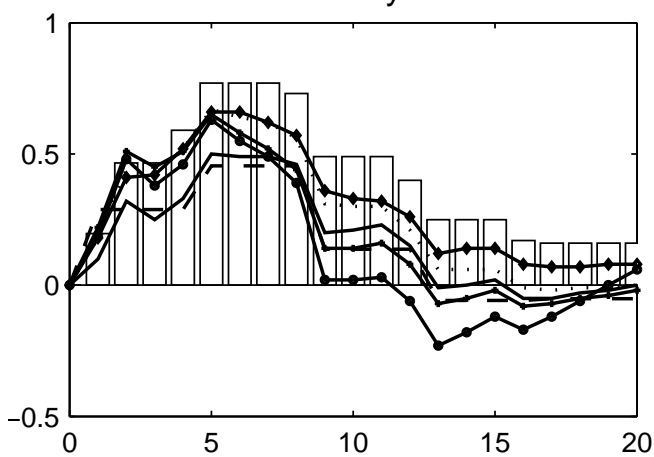

Two Years of Monetary Accommodation

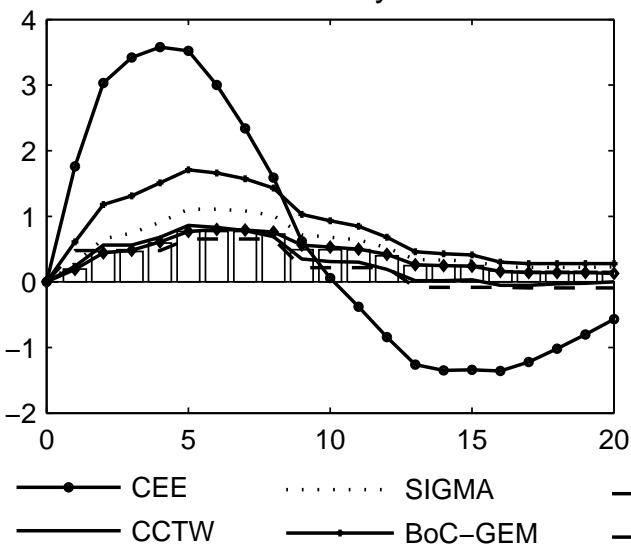

Europe

No Monetary Accommodation

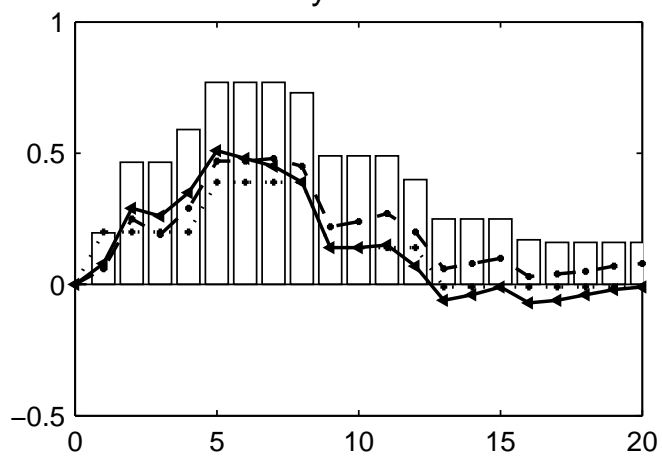

One Year of Monetary Accommodation

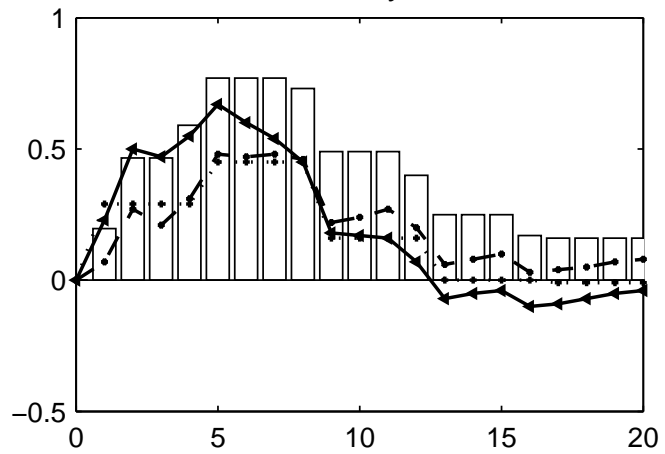

Two Years of Monetary Accommodation

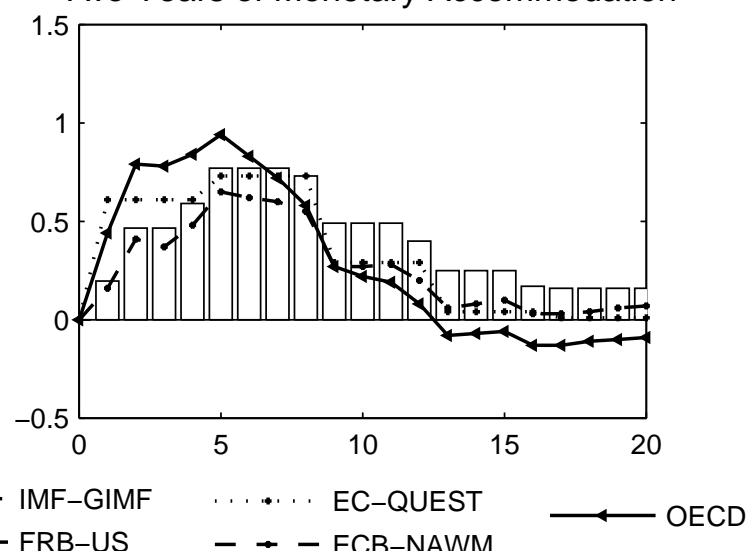

Notes: Horizontal axis represents time horizon in quarters. Units of the vertical axis are the percentage of GDP. Shown are estimated GDP effects of government purchases in the February 2009 U.S. stimulus legislation for nine macroeconomic models. The bars shown in each panel are identical and indicate the time profile of the planed ARRA government spending. CEE is the model of Christiano et al. (2011); CCTW is the model of Cogan et al. (2010); IMFGIMF is the IMF's Global Integrated Monetary and Fiscal Policy model; FRB-US is the Federal Reserve's US model; SIGMA is the Federal Reserve's two-country model; BoC-GEM is the Bank of Canada's Global Economy Model; ECQUEST is the European Commissions QUEST model; ECB-NAWM is the European Central Bank's New Area-Wide Model; OECD is the OECD's macroeconomic model. 
the models even predict an overall negative effect on GDP in the fourth year of the stimulus. The simulation outcome of the CCTW model lies in between the other outcomes displayed in the left panel of Figure 1. This finding provides further support of the CCTW results, in particular, since several of the other models incorporate a more detailed fiscal sector.

For the simulations shown in the second row of panels, nominal interest rates are held constant for one year and follow a model-specific rule thereafter. For the results shown in the third row, nominal interest rates are held constant for two years. These simulations illustrate the role of the degree of monetary accommodation in the effectiveness of fiscal stimulus. If nominal interest rates are initially held constant, fiscal multipliers increase. With one year of anticipated monetary accommodation, multipliers still remain below one in all of the models. With two years of monetary accommodation, the increase in GDP exceeds the increase in government spending a little bit in some of the models, due to crowding-in of private demand. There is one outlier. The CEE model exhibits a very large effect for two years, followed by a recession. As suggested in Christiano et al. (2011), government spending multipliers may be large in this model. Yet, all the other models considered by Coenen et al. (2012) imply much smaller effects on GDP. Thus, the larger multiplier effect in the CEE model is not just due to the monetary accommodation resulting in the presence of the zero bound. There are other features of the CEE model that lead to greater effectiveness of fiscal stimulus in this scenario. Coenen et al. (2012) suggest that the CEE model is an outlier, because it exhibits a much lower degree of price rigidity.

In sum, the large-scale comparison exercise confirms the cautionary assessment of the likely impact of ARRA provided by Cogan et al. (2010) and helps identify outliers. It would certainly have been useful to have such a large-scale comparison available in 2009 in order to provide policy advice to the Obama Administration and the members of U.S. Congress. Thus, it is of great interest to explore how model comparisons can be implemented more easily and more frequently whenever such analysis can help inform policy makers in real time.

\section{A systematic approach to model comparison}

One important goal of model comparison exercises is to identify policy implications that are due to different structural features of the respective models. Yet, quantitative simulation results may also differ because the economic concepts and variables to be compared are not defined consistently across models. Furthermore, different outcomes may be due to different assumptions about policy rather than different structures of the economy. This section briefly describes how macroeconomic models can be augmented systematically with a few equations to produce comparable objects concerning policy implications for key macroeconomic aggregates, while keeping the total number of modifications quite small. This formal approach is elaborated on in Wieland et al. (2012).

\section{Notation for a general nonlinear model.}

The following notation is used to define a general nonlinear model of the economy. The su- 
perscript $m=(1,2,3, \ldots, M)$ denotes the equations, variables, parameters and shocks of a specific model $m$ that is to be included in the comparison. These model-specific objects need not be comparable across models. They are listed in Table 2. In the computational implementation, $m$ corresponds to an abbreviated model name rather than simply a number.

Table 2: Model-Specific Variables, Parameters, Shocks and Equations

\begin{tabular}{ll}
\hline Notation & Description \\
\hline$x_{t}^{m}$ & endogenous variables in model $m$ \\
$x_{t}^{m, g}$ & policy variables in model $m$ (also included in $\left.x_{t}^{m}\right)$ \\
$\eta_{t}^{m}$ & policy shocks in model $m$ \\
$\varepsilon_{t}^{m}$ & other economic shocks in model $m$ \\
$g_{m}()$. & policy rules in model $m$ \\
$f_{m}()$. & other model equations in model $m$ \\
$\gamma^{m}$ & policy rule parameters in model $m$ \\
$\beta^{m}$ & other economic parameters in model $m$ \\
$\Sigma^{m}$ & covariance matrix of shocks in model $m$ \\
\hline
\end{tabular}

Two types of model equations are distinguished. Policy rules are denoted by $g_{m}($.$) while all$ other equations and identities are denoted by $f_{m}($.$) . Together, they determine the endogenous$ variables denoted by the vector $x_{t}^{m}$. These variables are functions of each other, of model-specific shocks, $\left[\begin{array}{cc}\varepsilon_{t}^{m} & \eta_{t}^{m}\end{array}\right]$, and of model-specific parameters $\left[\begin{array}{ll}\beta^{m} & \gamma^{m}\end{array}\right]$. A particular model $m$ is then defined by:

$$
\begin{aligned}
& E_{t}\left[g_{m}\left(x_{t}^{m}, x_{t+1}^{m}, x_{t-1}^{m}, \eta_{t}^{m}, \gamma^{m}\right)\right]=0 \\
& E_{t}\left[f_{m}\left(x_{t}^{m}, x_{t+1}^{m}, x_{t-1}^{m}, \varepsilon_{t}^{m}, \beta^{m}\right)\right]=0
\end{aligned}
$$

The superscript $m$ refers to the version of the respective model originally presented by its authors. The model may include current values, lags and the expectation of leads of endogenous variables. In equations (1) and (2) the lead- and lag-lengths are set to unity for notational convenience. Additional leads and lags can be accommodated with auxiliary variables. Even so, our software implementation does not restrict the lead- and lag-lengths of participating models.

The model may also include innovations that are random variables with zero mean and covariance matrix, $\Sigma^{m}$ :

$$
\begin{aligned}
E\left(\left[\eta_{t}^{m} \varepsilon_{t}^{m}\right]^{\prime}\right) & =0 \\
E\left(\left[\eta_{t}^{m \prime} \varepsilon_{t}^{m \prime}\right]^{\prime}\left[\eta_{t}^{m \prime} \varepsilon_{t}^{m \prime}\right]\right) & =\Sigma^{m}=\left(\begin{array}{cc}
\Sigma_{\eta}^{m} & \Sigma_{\eta \varepsilon}^{m} \\
\Sigma_{\eta \varepsilon}^{m} & \Sigma_{\varepsilon}^{m}
\end{array}\right)
\end{aligned}
$$

We refer to innovations interchangeably as shocks. Some models include serially correlated economic shocks that are themselves functions of random innovations. In our notation, such serially correlated economic shocks would appear as elements of the vector of endogenous variables $x_{t}^{m}$, 
only the innovations would appear as shocks. Equation (4) distinguishes the covariance matrices of policy shocks and other economic shocks as $\Sigma_{\eta}^{m}$ and $\Sigma_{\varepsilon}^{m}$. The correlation of policy shocks and other shocks is typically assumed to be zero, $\Sigma_{\eta \varepsilon}^{m}=0$.

Introducing common variables, parameters, equations and shocks.

In order to compare policy implications from different models, it is necessary to define a set of comparable variables, shocks and parameters. They are common to all models considered. Policies can then be expressed in terms of such common parameters, variables and policy shocks, and their consequences can be calculated for a set of common endogenous variables. Our notation for comparable endogenous variables, policy instruments, policy shocks, policy rules and parameters is given in Table 3

Table 3: Comparable Common Variables, Parameters, Shocks and Equations

\begin{tabular}{ll}
\hline Notation & Description \\
\hline$z_{t}$ & common variables in all models \\
$z_{t}^{g}$ & common policy variables in all models (also included in $z_{t}$ ) \\
$\eta_{t}$ & common policy shocks in all models \\
$g()$. & common policy rules \\
$\gamma$ & common policy rule parameters \\
\hline
\end{tabular}

Every model to be included in the comparison has to be augmented with common variables, parameters and shocks. Augmenting the model requires adding some equations. These additional equations serve to define the common variables in terms of model-specific variables. We denote these definitional equations or identities by $h_{m}($.$) . They are necessarily model-specific. Addition-$ ally, the original model-specific policy rules need to be replaced with common policy rules. Of course, these common rules could be defined generally enough such that they nest many of the model-specific policy rules. Furthermore, there are many interesting questions that would require comparing model implications for common variables of interest when policy follows the respective model-specific rule. An example is provided in Section 5.3

All the other equations, variables, parameters and shocks may be preserved in the original notation of the model authors. Consequently, the augmented model consists of three components: (i) the common policy rules, $g($.$) , expressed in terms of common variables, z_{t}$, policy shocks, $\eta_{t}$, and policy parameters, $\gamma$; (ii) the model-specific definitions of common variables in terms of original model-specific endogenous variables, $h_{m}($.$) , with parameters \theta^{m}$; (iii) the original set of model-specific equations $f_{m}($.$) that determine the endogenous variables. It corresponds to:$

$$
\begin{aligned}
E_{t}\left[g\left(z_{t}, z_{t+1}, z_{t-1}, \eta_{t}, \gamma\right)\right] & =0 \\
E_{t}\left[h_{m}\left(z_{t}, x_{t}^{m}, x_{t+1}^{m}, x_{t-1}^{m}, \theta^{m}\right)\right] & =0 \\
E_{t}\left[f_{m}\left(x_{t}^{m}, x_{t+1}^{m}, x_{t-1}^{m}, \varepsilon_{t}^{m}, \beta^{m}\right)\right] & =0
\end{aligned}
$$


Models augmented accordingly are ready for comparing policy implications. For example, it is then straightforward to compare the consequences of a particular policy rule for the dynamic behavior of consistently defined endogenous variables across models. This approach requires only a limited number of common elements. The rest of each model remains unchanged in the authors' original notation. This includes the variable names and definitions of endogenous variables, $x_{t}^{m}$, the other economic shocks $\varepsilon_{t}^{m}$, the equations $f_{m}($.$) with model parameters \beta^{m}$ and the covariance matrix of shocks $\Sigma_{\varepsilon}^{m}$. The covariance matrix of policy shocks $\Sigma_{\eta}$ may be treated as an element of the vector of policy parameters or set to zero.

Wieland et al. (2012) provide some concrete examples for the model augmentation step, which includes setting up the additional definitional equations, $h_{m}($.$) , and determining their parameters,$ $\theta^{m}$. The subsequent steps in comparing policy implications consist of solving the augmented models, constructing appropriate objects for comparison and computing a metric that quantifies the differences of interest.

\section{Computing comparable policy implications.}

Solving the augmented nonlinear structural model defined by equations (5), (6) and (7) involves expressing the expectations of future variables in terms of currently available information. To this end, one needs to define how expectations are formed. Our computational implementation and model archive, Macroeconomic Model Data Base, includes models using several different assumptions. While most of the models are solved under the assumption of rational model-consistent expectations, several models can also be solved under the assumption of adaptive learning in expectations as in Slobodyan and Wouters (2012). Other assumptions regarding expectations formation include the sticky-information model of Mankiw and Reis (2007) with staggered information sets of otherwise rational expectations and VAR-based expectations used in Orphanides (2003) and in a version of the Federal Reserve's FRB-US model.

Here, we proceed under the assumption of rational expectations. The solution step involves checking for existence and uniqueness of equilibrium. For linear models one can use the BlanchardKahn conditions. For nonlinear models one may have to rely on search by numerical methods. The solution of the structural model is given by a set of reduced-form equations:

$$
\begin{aligned}
z_{t} & =k_{z}\left(z_{t-1}, x_{t-1}^{m}, \eta_{t}, \varepsilon_{t}^{m}, \kappa_{z}\right) \\
x_{t}^{m} & =k_{x}\left(z_{t-1}, x_{t-1}^{m}, \eta_{t}, \varepsilon_{t}^{m}, \kappa_{x}\right)
\end{aligned}
$$

If the structural model is nonlinear, the reduced-form equations will also be nonlinear. $\left(\kappa_{z}, \kappa_{x}\right)$ denote the reduced-form parameters. They are complicated functions of the structural parameters, $\beta^{m}$, the policy parameters, $\gamma$, and the covariance matrix, $\Sigma^{m}$. Nonlinear models may be solved approximately by means of numerical methods, for example, perturbation-based, projection-based or two-point-boundary-value algorithms (see Judd (1998), Fair and Taylor (1983), Collard and Juillard (2001)). When the model is first be linearized around a deterministic steady state, ei- 
ther analytically or numerically, a range of methods are available for computing the solution to the linear system of expectational equations. These methods include the generalized eigenvalueeigenvector method (see Uhlig (1995)), generalized Schur decomposition (see Klein (2000)), QZ decomposition (see Sims (2001)), the undetermined coefficients method (see Christiano (2002)), or the Anderson-Moore algorithm for solving linear saddle point models (see Anderson and Moore (1985)).

The reduced form solution of the augmented nonlinear model can then be used to obtain particular objects for comparison defined in terms of comparable variables. With regard to policy implications, one object of interest could be the impact of a policy shock and its transmission to key macroeconomic aggregates. This object corresponds to the dynamic response of a particular common variable (an element of $z$ ) to a policy shock $\eta_{t}$, conditional on a certain common policy rule, $g\left(z_{t}, z_{t+1}, z_{t-1}, \eta_{t}, \gamma\right)$. Such impulse response functions describe the isolated effect of a single shock on the dynamic system holding everything else constant. Other objects of interest for comparing policy implications would be the unconditional variances and serial correlation functions. Finally, one may compute suitable metrics for measuring the distance between two or more models. Such metrics could be the absolute difference of the unconditional variances or the absolute difference of the impact effects of policy shocks under different models.

\section{Practical problems and a new platform}

Large-scale macroeconomic model comparison exercises have been relatively rare. These exercises are costly because they typically involve multiple meetings of several teams of model developers, with each team analyzing the policy scenarios in its own model. At the same time, the number of policy scenarios studied in these exercises has been limited. In this section, we review some practical problems that have hampered easy and frequent use of model comparison. We also report on the experience with strategies employed in the construction of the Macroeconomic Model Data Base to overcome these problems. At this point, there are 66 models available for easy use by individual researchers and students. It is straightforward to include new models and compare their policy implications to existing benchmarks.

\subsection{Replication, computational implementation and model archiving}

\section{Replication.}

The first practical problem that arises if a researcher wishes to compare her macroeconomic model to those of others is how to obtain sufficient information about their models to conduct her own analysis. Replicability is a basic scientific principle. The web-course "Understanding science 101" at UC Berkeley describes this principle as follows:

"Scientists aim for their studies' findings to be replicable - so that, for example, an 
experiment testing ideas about the attraction between electrons and protons should yield the same results when repeated in different labs. Similarly, two different researchers studying the same dinosaur bone in the same way should come to the same conclusions regarding its measurements and composition. This goal of replicability makes sense. After all, science aims to reconstruct the unchanging rules by which the universe operates, and those same rules apply, 24 hours a day, seven days a week, from Sweden to Saturn, regardless of who is studying them. If a finding can't be replicated, it suggests that our current understanding of the study system or our methods of testing are insufficient."

Unfortunately, however, there is no general practice guaranteeing replicability of macroeconomic models that are solved and simulated by means of computational methods. This state of the field is somewhat surprising compared to other fields of economics. In economic theory it is standard that articles in scientific economic journals provide sufficient detail on mathematical derivations and proofs such that academics and advanced students can replicate the analysis. In econometrics new methods and estimators are fairly quickly implemented in software packages such as EVIEWS, RATS, SAS, GAUSS and others. Thus, new econometric tools are not only spread to academic researchers and students but widely used by practitioners in many fields of applied economic analysis. In the last two decades, macroeconomic modeling has benefited from a similar development with regard to numerical techniques for solving and estimating models with rational expectations. Initially, individual researchers have made particular toolkits available that have been adopted by many others in their work. Over the years, the software package DYNARE developed by Michel Juillard and collaborators has gained more and more users and contributors such that it has become a widely used tool for macroeconomic model solution and estimation (see Juillard (2001) and Adjemian et al.(2011)). While new techniques for model solution and estimation can now be easily employed by academics, students and practitioners almost as "black box" systems, this is not true for most of the many new macroeconomic models.

The following problems can arise when one attempts to replicate macroeconomic models presented in economic journals:

1. The published article does not contain all the equations needed to write the model code for replicating the analysis presented in the article. Typically, journals are not willing to devote space to present all the information that is needed. Typically, the models are quite complex. Thus, errors may occur in transcribing model equations that were successfully implemented in computer code to the text file for the article.

2. The published article does not contain all the parameter values or steady-state values needed to replicate the model simulations reported in the article.

3. The code for replicating the model is not available from the journal's website. While many journals provide options for online archiving of supplementary materials only a few have 
the capacity to insist in every case that authors provide a reliable version of their code.

4. The code is not available from the authors' website and authors are not replying to requests for the code.

5. The code is available but the software needed to simulate is unavailable to individual researchers because its price is high and it is only used at large institutions. An example is the TROLL software used at some policy making institutions.

6. The code is available but the simulation results it delivers differ from the results published in the article. Such inconsistencies may simply be due to differences in the date of the version used for preparing the results shown in the article and the version made available for replication.

7. The code that is available does not contain sufficient description and explanations such that it is easily understood by users.

8. The software platform for which the code has been written has been updated such that the code cannot be executed successfully anymore on this platform.

9. The researcher attempting replication makes errors in his implementation of the model.

10. The published model cannot be replicated correctly because the derivation of the equations or their implementation in computer code contains errors. Given the complex nature of computational implementation of medium- and large-scale macroeconomic models, such errors are to be expected and can happen to the most meticulous scholars. It is useful to recognize and correct them so as to make it easier for other researchers to build on this work.

These difficulties are not unique to macroeconomic modeling. Replication in reference to computations is more commonly known as "reproducible research" and forms the subject of an expanding literature in computer science, statistics and related fields of application (see for example Fomel and Claerbout (2009), Donoho (2010), Freire et al. (2012) and Sandve et al. (2013)). Stanford statistician Donoho characterizes the central problem in these words:

"an article about computation result is advertising, not scholarship. The actual scholarship is the full software environment, code and data, that produced the result.'2

We have pursued the following strategies for replicating models to be included in our model comparison software:

\footnotetext{
${ }^{2}$ Noteworthy, this quote is inspired by Claerbout (1994). Very similar ideas are expressed much earlier by A. Williams (see White (1978)).
} 
1. The ideal case is that authors or other users of the model provide the code describing the model and integrate it themselves in MMB. Generally, authors can expect wider dissemination, use and citation of their work by other researchers if they make their code available in an easy-to-use format. We have also found that policy making institutions such as central banks and international organisations have become very open towards making their models available, at least those versions that economists from these institutions have circulated in working papers or used for publications in scientific journals.

2. The next best scenario is when model authors provide the complete code that replicates the findings reported in their article and remain available for answering questions of research assistants in Frankfurt who integrate the model in MMB.

3. Research assistants in the Frankfurt MMB team have replicated a number of models using software made available on journal or author websites.

4. We teach advanced Ph.D. courses that focus on a particular area of new model development. A team of two or three students receives the task of presenting a paper from the literature, replicating the model and integrating the model in MMB. This approach has proved quite successful in terms of training Ph.D. students in model building and getting them to the research frontier, where they can work on extending existing models for new applications. Whether they succeed in replicating the model often depends on whether they receive feedback from model authors on problems or missing items. Students give presentations on the original paper and the technical replication and they also prepare a replication report.

5. Once a model has been replicated, we make the files documenting the replication available for download on the MMB website (http://www.macromodelbase.com) as shown in Figure 2 . The replication package is offered separate from the comparison software itself. It is not augmented for model comparison and remains as close as possible to the authors' original code or article. A readme file and graphics files make reference to the specific original research findings and provide information on how we came to matching the authors' work.

In total MMB 2.1 makes available 66 models. Taking into account that some of them are simple variants of one model, MMB 2.1 includes 57 quite distinct models. Out of 57 models, about 12 models were made ready for integration by the original authors or other researchers. 31 models were implemented by the MMB team in Frankfurt in cooperation with the original authors, and the remainder were integrated on the basis of course work by Ph.D. students.

\section{Computational implementation.}

In terms of implementing the model comparison approach outlined in the previous section computationally, there are choices to be made regarding computer language as well as model solution and simulation methods. Furthermore, problems to be dealt with concern the compatibility with earlier or subsequent version of the respective software solutions and operating systems. 
Figure 2: MMB WEBSITE

The Macroeconomic Model Data Base

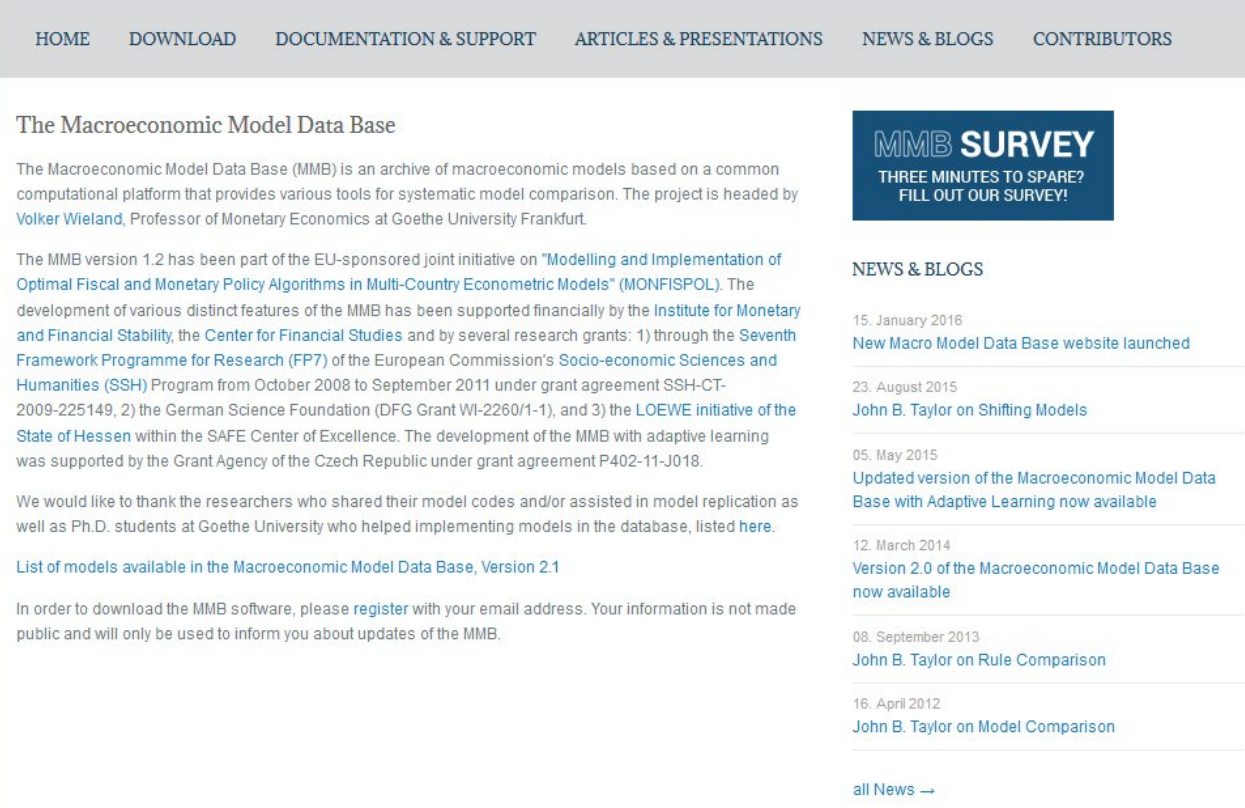

Most academic researchers in the area of macroeconomic model building have adopted MATLAB as their preferred high-level programming language. This choice concerns specifically the recent development of dynamic stochastic general equilibrium (DSGE) models in the real business cycle and New Keynesian literatures. MATLAB-the name is derived from MATrix LABoratoryis a commercial software product of MATHWORKS Inc. It is fairly widely used in engineering, physics, economics and other fields applying computational methods. This software product is expensive but there are discounts for student licences. Also, there exists a freeware software GNU OCTAVE that is largely compatible with the proprietary MATLAB software. Thus, executables that run on MATLAB can presumably be executed on OCTAVE without needing major modifications. Competing software packages such as GAUSS or MATHEMATICA are not as popular in macroeconomic modelling but offer advantages in econometric or symbolic methods, respectively.

Developers of numerical solution methods for macroeconomic models with rational expectations have written routines that are MATLAB executables for a long time. Over recent years, the free software package DYNARE has been adopted by many researchers in academia, central banks and international organisations that are working in the field of macroeconomic modeling (see www.dynare.org). DYNARE runs on MATLAB but can also be used with OCTAVE. There is a growing community of researchers that is contributing freely available routines for solution, 
estimation and optimization to the DYNARE environment. Many central banks and international organisations also employ the software system TROLL for simulating models used in policy formulation. TROLL is a commercial software with features that make it easy to manage large data sets.

MMB has been developed as free software to be used with DYNARE and MATLAB. Models are defined in the syntax needed for DYNARE. It should also be possible to use the first version of MMB 1.2 and DYNARE with the free software OCTAVE. Yet, so far we have not had the resources to ensure that MMB 2.0 and 2.1 are OCTAVE compatible. MMB 2.0 has been extended with graphical user interfaces (GUI) to improve user friendliness. At this point, GUI facilities are apparently not yet available on OCTAVE, thus restricting MMB 2.0 to MATLAB environments. A MAC compatible version of MMB 1.2 is available for download thanks to the contribution of Raymond Hawkins from the University of Arizona.

\subsection{User friendliness and a MATLAB-based platform for comparative analysis}

\section{User friendliness.}

The first version of MMB 1.2 was intended for researchers that work on building macroeconomic models. MMB 2.0 and updates are meant to be accessible to a wider group of interested professional economists in the public and private sector and to students of macroeconomics. Thus, we have built graphical user interfaces that make it easier to simulate a wide variety of scenarios with any of the models included in the archive.

First, the user can choose among different applications such as the comparison of different models under a common policy rule, (One policy rule, many models), or an in-depth analysis of one specific model under different policy rules, (One model, many policy rules). Then he is offered a menu of choices concerning models, policy rules, simulation scenarios and output formats.

For example, the menu for One policy rule, many models is shown in Figure 3 . This menu offers options to conduct comparisons across models under the assumption that the central bank in each model implements the same interest rate rule. It gives access to a software implementation of the mathematical representation of model comparison in Section 3

On the left-side of the menu the user can choose multiple models by clicking on the respective boxes. Models are grouped under different categories such as calibrated New Keynesian models, estimated models of the U.S. economy, estimated models of the euro area economy, models of other economies such as Canada, Chile, Brazil or HongKong and finally several multi-country models. A button on the bottom right of the menu titled "Models description" leads to PDF file with further information on the models included in the archive. On the top right side, there is a section for choosing a common policy rule from a list of rules. Alternatively, the user can pick coefficients for the common rule in a sub-menu. Furthermore, there are various options for generating simulation output such as unconditional variances, autocorrelation functions and impulse response functions to monetary and fiscal policy shocks. 
Figure 3: Modelbase Menu: One Policy RUle, Many MOdels

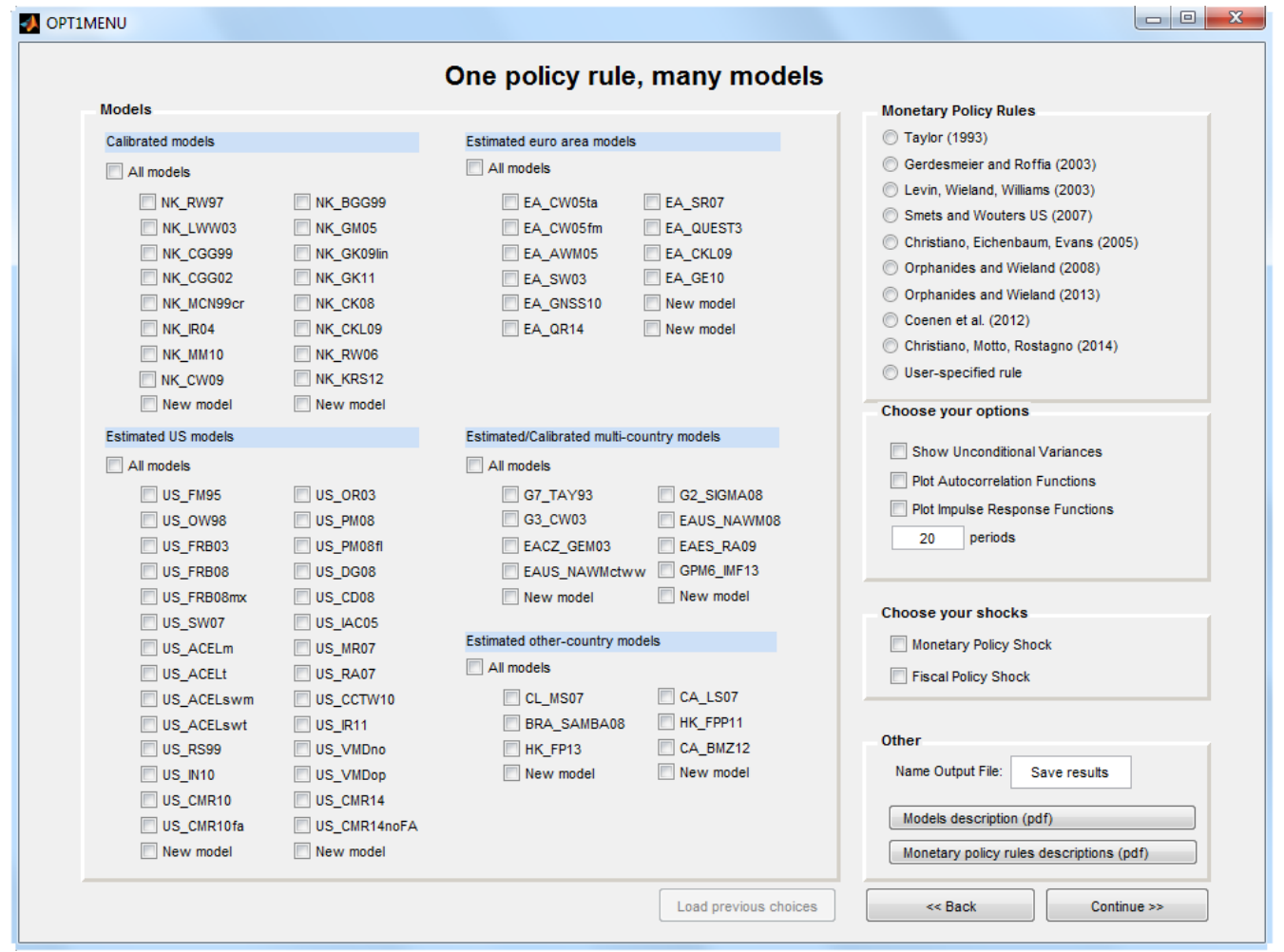

\section{Common and model-specific policy rules.}

The comparison using a common policy rule makes it possible to identify differences in policy implications that are due to differences in model structure and parameter estimates. Yet, there are other interesting questions one might want to ask. For example, it may be of interest to explore the dynamics of one particular model under a variety of different policy rules in more detail. And there are questions that would require simulating each model under the original policy rule estimated or calibrated by the model authors. For example, one would use the model-specific rules if one wants to compare the fit of each model to the data, if one wants to identify the typical empirical response to a particular model-specific shock, or if one wants to compare forecasts obtained from different models.

The application (One model, many policy rules) allows a thorough investigation of the properties of a single model and can be used to compare the implications of a variety of policy rules in this model. The user can only choose one model at a time, but multiple policy rules. It is possible to list the structural shocks in each model and simulate impulse responses for some or all of them under the different rules. In addition to the list of rules and the user-specified rule, the rules menu also includes the model-specific rule estimated or calibrated by the original model authors as long as the model-specific rule can be written in terms of MMB common variables. 
Figure 4: Modelbase Menu: One MOdel, MANY POlicy RUles

\begin{tabular}{|c|c|c|c|c|c|}
\hline \multicolumn{4}{|l|}{ OPT2MENU } & \multicolumn{2}{|c|}{\begin{tabular}{|l|l|} 
& $\mid$ \\
\end{tabular}} \\
\hline \multicolumn{6}{|c|}{ One model, many policy rules } \\
\hline \multicolumn{2}{|l|}{ Models } & \multirow{2}{*}{\multicolumn{2}{|c|}{ Estimated euro area models }} & \multicolumn{2}{|c|}{ Monetary Policy Rules } \\
\hline \multicolumn{2}{|l|}{ Calibrated models } & & & \multirow{2}{*}{\multicolumn{2}{|c|}{$\begin{array}{l}\square \text { Taylor (1993) } \\
\square \text { Gerdesmeier and Roffia (2003) }\end{array}$}} \\
\hline NK_RW97 & NK_BGG99 & EA_Cw05ta & EA_SR07 & & \\
\hline NK_LWWN03 & NK_GM05 & EA_CW05fm & O EA_QUEST3 & \multicolumn{2}{|c|}{$\square$ Levin, Wieland, Williams (2003) } \\
\hline NK_CGG99 & NK_GK09lin & EA_AWMO5 & EA_CKLO9 & \multicolumn{2}{|c|}{$\square$ Smets and Wouters US (2007) } \\
\hline NK_CGG02 & NK_GK11 & EA_SW03 & EA_GE10 & \multicolumn{2}{|c|}{$\square$ Christiano, Eichenbaum, Evans (2005) } \\
\hline NK_MCN99cr & NK_CK08 & FA GNSS10 & New model & \multicolumn{2}{|c|}{$\square$ Orphanides and Wieland (2008) } \\
\hline NK_IR04 & NK_CKL09 & EA_GNSSTO & New mivot & \multirow{2}{*}{\multicolumn{2}{|c|}{$\begin{array}{l}\square \text { Orphanides and Wieland (2013) } \\
\square \text { Coenen et al. (2012) }\end{array}$}} \\
\hline NK_MM10 & NK_RW06 & EA_QR14 & New model & & \\
\hline NK_CW09 & NK_KRS12 & & & \multirow{2}{*}{\multicolumn{2}{|c|}{$\begin{array}{l}\square \text { Christiano, Motto, Rostagno (2014) } \\
\square \text { User-specified rule }\end{array}$}} \\
\hline New model & New model & & & & \\
\hline \multicolumn{2}{|l|}{ Estimated US models } & \multicolumn{2}{|c|}{ Estimated/Calibrated multi-country models } & \multirow{2}{*}{\multicolumn{2}{|c|}{$\begin{array}{l}\square \text { Model specific rule } \\
\square \text { All available rules }\end{array}$}} \\
\hline US_FM95 & US_OR03 & G7_TAY93 & G2_SIGMA08 & & \\
\hline us_ow98 & US_PM08 & G3_CW03 & EAUS_NAWM08 & \multicolumn{2}{|c|}{ Choose your options } \\
\hline US_FRB03 & us_PM08fl & EACZ_GEM03 & EAES_RA09 & \multicolumn{2}{|c|}{$\square$ Show Unconditional Variances } \\
\hline US_FRB08 & US_DG08 & EAUS_NAWMctww & GPM6_IMF13 & \multicolumn{2}{|c|}{$\square$ Plot Autocorrelation Functions } \\
\hline US_FRB08mx & US_CD08 & New model & New model & \multicolumn{2}{|c|}{$\square$ Plot Impulse Response Functions } \\
\hline us_sw07 & us_laC05 & \multirow{2}{*}{\multicolumn{2}{|c|}{ Estimated other-country models }} & \multirow{2}{*}{\multicolumn{2}{|c|}{20 periods }} \\
\hline US_ACELm & US_MR07 & & & & \\
\hline US_ACELt & US_RA07 & CL_MS07 & CA_LS07 & \multicolumn{2}{|c|}{ Choose your shocks } \\
\hline O US_ACELswm & us_cctw10 & BRA_SAMBA08 & HK_FPP11 & \multicolumn{2}{|c|}{ Monetary Policy Shock } \\
\hline US_ACELswt & us_IR11 & HK_FP13 & CA_BMZ12 & \multicolumn{2}{|c|}{ Model Specific Shocks } \\
\hline US_RS99 & us_vidno & New model & New model & \multirow{2}{*}{\multicolumn{2}{|c|}{ Other }} \\
\hline us_IN10 & us_vMDop & & & & \\
\hline US_CMR10 & US_CMR14 & & & \multicolumn{2}{|c|}{ Name Output File: Save results } \\
\hline Us_CMR10fa & US_CMR14noFA & & & \multicolumn{2}{|c|}{ Models description (pdf) } \\
\hline New model & New model & & & \multicolumn{2}{|c|}{ Monetary policy rules descriptions (pdf) } \\
\hline & & & Load previous choices & $\ll$ Back & Continue $\gg$ \\
\hline
\end{tabular}

How to include your own model in MMB.

It is fairly straightforward to include additional models in the archive. A detailed description of the necessary steps is provided in the MMB User Guide that can be downloaded along with the MMB software. Thus users can easily integrate their own model for comparison with the models in the archive. The new model can be assigned a button in the graphical user interface. If users send their model file to the model base team in Frankfurt it can also be included on the publicly available archive.

The complete process of augmenting a model has been described formally in Section 3 If modelers have already simulated their model using DYNARE, they only need to make a few adjustments and additions to the DYNARE model in order to integrate their model in the MMB software. To illustrate this process, Figures 5 and 6 present the central elements of the DYNARE model file with the New Keynesian model by Rotemberg and Woodford (1997) (NK_RW97) in MMB. A typical model file is comprised of the preamble block, in which variables and parameters are initialized, and the model block 3

With regard to the preamble of their model file, contributors simply need to copy and paste

\footnotetext{
${ }^{3}$ For more detailed explanations, please refer to section 1.4 in the MMB User Guide available online at www.macromodelbase.com.
} 
in the common variables, common policy shocks and common policy parameters from another MMB model file. The lines of code that need to be pasted in are shown between starred lines in the preamble section in Figures 5 . They are the same for all MMB model files.

The augmented model block consists of three parts: (i) the common policy rules $(g($.$) in equa-$ tions 5p; (ii) the definitional equations $\left(h_{m}(\right.$.$) in equations 6); (iii) the original model equations$ $\left(f_{m}(\right.$.$\left.) in equations 7\right)$. Including the common policy rules is simply another "copy and paste" operation (see lines 63 to 75 in Figure 6). Of course, the model-specific monetary policy rule then needs to be commented out (see line 87). The only step that requires more knowledge of the original model concerns adding the definitions of the common variables in terms of model specific variables to the code. Table 4 describes the relevant common variables. The resulting definitional equations in the case of the NK_RW97 model can be found in lines 54 to 59 of Figure 6.

Table 4: Comparable Common Variables in MMB

\begin{tabular}{lll}
\hline Notation & Variable name & Description \\
\hline$i_{t}^{z}$ & interest & annualized quarterly money market rate \\
$g_{t}^{z}$ & fispol & discretionary government purchases (share in GDP) \\
$\pi_{t}^{z}$ & inflation & year-on-year rate of inflation \\
$p_{t}^{z}$ & inflationq & annualized quarter-to-quarter rate of inflation \\
$y_{t}^{z}$ & output & quarterly real GDP \\
$q_{t}^{z}$ & outputgap & quarterly output gap (deviation from flex-price level) \\
\hline
\end{tabular}


Figure 5: StRUCture of THE MOdel FILE FOR ROTEMBERG AND WOODFORD (1997) (NK_RW97) : THE PREAMbLE

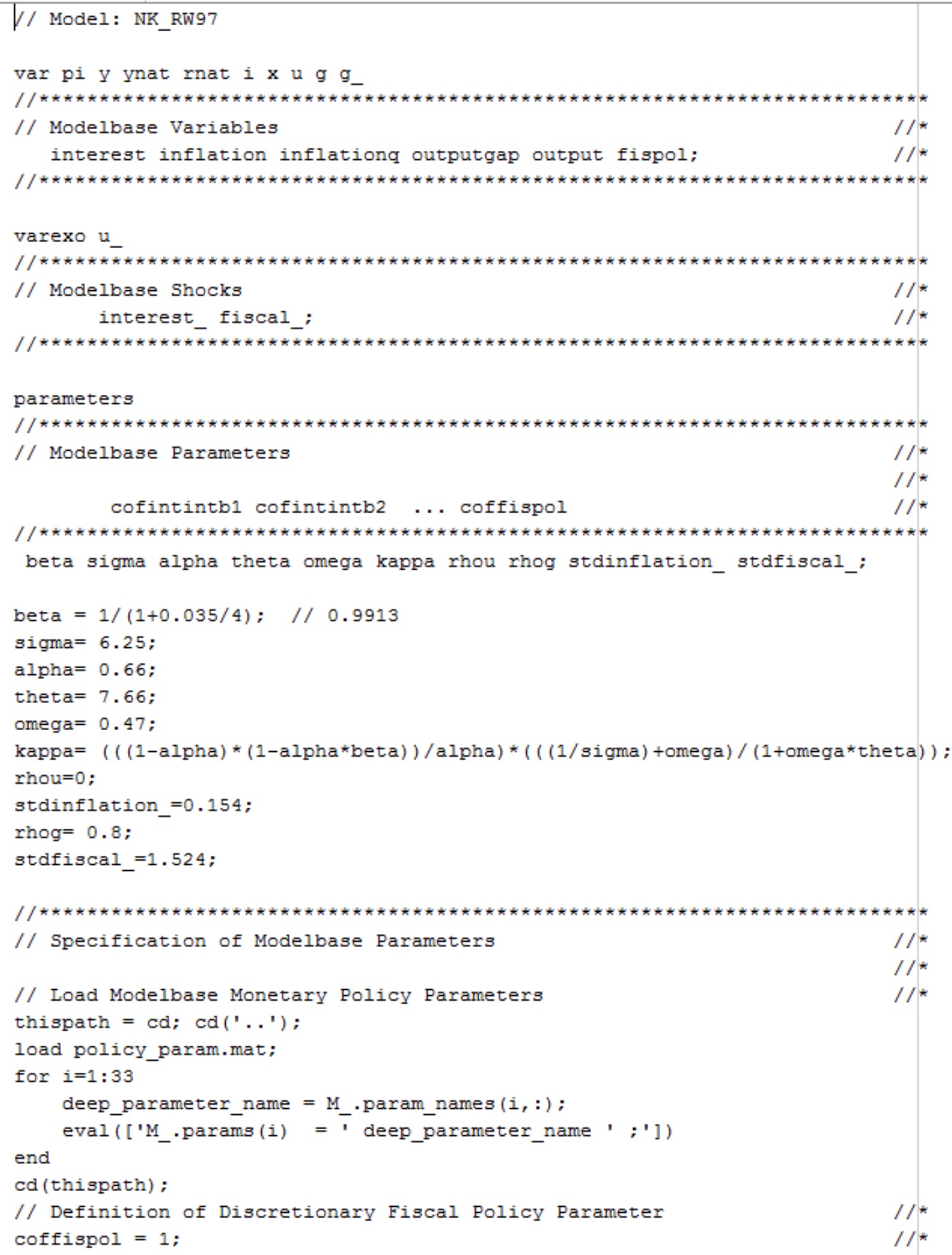


Figure 6: Structure of THE MOdel File FOR ROTEMBERG AND WOODFORD (1997) (NK_RW97) : THE MODEL BLOCK

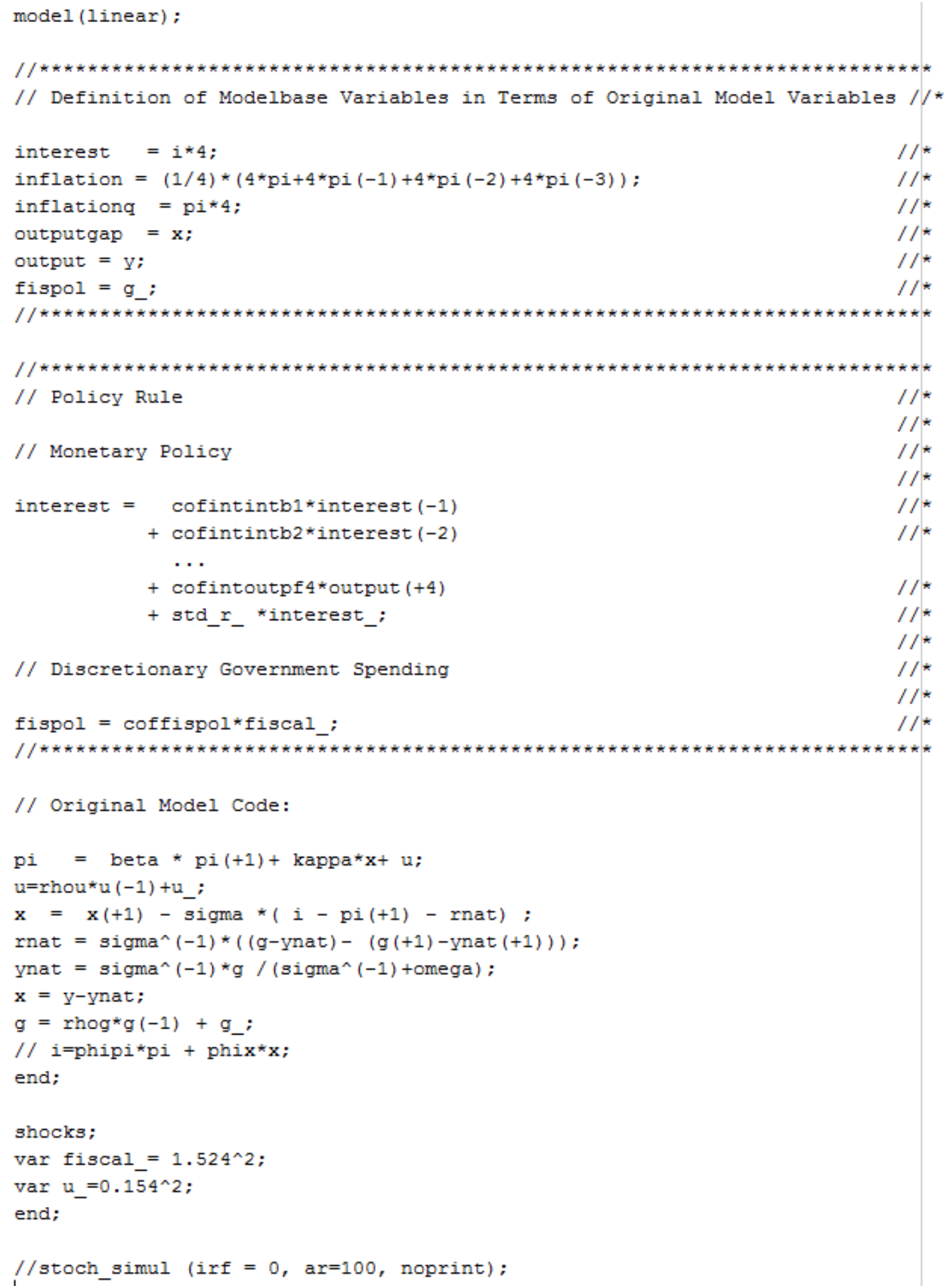




\section{Comparing fiscal and monetary policy transmission using the new platform}

The Macroeconomic Model Data Base offers individual users many options for comparing model structures and policy implications and for exploring a particular model in great detail. There is no need for bringing together teams of model builders each analyzing its own model. In the following, we present three exercises that are easy to carry out and serve to showcase the potential usefulness of the MMB technology to researchers and economists at policy institutions.

The first exercise shows how researchers can use it to evaluate the sensitivity of policy implications to key model parameters. Specifically, it reviews the importance of Keynesian consumers and monetary policy accommodation for fiscal stimulus effects in one of the models participating in the Coenen et al. (2012) comparison study. The second exercise extends the study of Taylor and Wieland (2012) on monetary policy transmission across earlier and more recent generations of structural macro models by including the medium-size New Keynesian model with financial frictions and risk shocks that Christiano et al. (2014) have recently estimated for the U.S. economy. Finally, the third exercise shows how to conduct cross-country comparisons and illustrates the use of model-specific rules in order to measure model uncertainty about policy effects.

\subsection{Effects of fiscal stimulus: Sensitivity to structural parameters}

The large-scale comparison study of Coenen et al. (2012) has highlighted the importance of monetary policy accommodation for Keynesian fiscal multiplier effects (see Section 2.2). Furthermore, the models participating in this study differed in terms of a relevant structural feature in this regard, namely the relative importance of Keynesian consumers that make decisions based on current income and Friedman-Modigliani permanent-income consumers that make forward-looking decisions based on life-time income. Here, we show how MMB users can evaluate the sensitivity of fiscal policy effects to the parameters governing household consumption choices and central bank reactions. To this end, we consider one of the models participating in the Coenen et al. (2012) study, namely the US_CCTW10 model of Cogan et al. (2010).

In terms of fiscal shock, we look at the effects of a surprise increase in government purchases that fades out gradually according to an autoregressive process. The shock is implemented as a common policy shock in MMB, that is, an element of the common shock vector $\eta_{t}$ introduced in Section $3^{4}$ As a consequence, government purchases increase on impact by 1 percent of GDP and then return slowly towards the original level.

\section{Parameter sensitivity analysis: Share of rule-of-thumb consumers}

The US_CCTW10 model extends the Smets and Wouters (2007) model with Keynesian-style rule-of-thumb households. These households simply consume all current disposable income. Us-

\footnotetext{
${ }^{4}$ At this point, the autoregressive parameter remains model-specific as an element of the parameter vector $\beta_{m}$. Yet, in other exercises we show how to consider common autoregressive parameters.
} 
Figure 7: IMPULSE RESPONSES TO AN EXPANSIONARY FISCAL POLICY SHOCK IN THE US_CCTW10 MODEL FOR ALTERNATIVE SHARES OF THE RULE-OF-THUMB HOUSEHOLDS

GDP
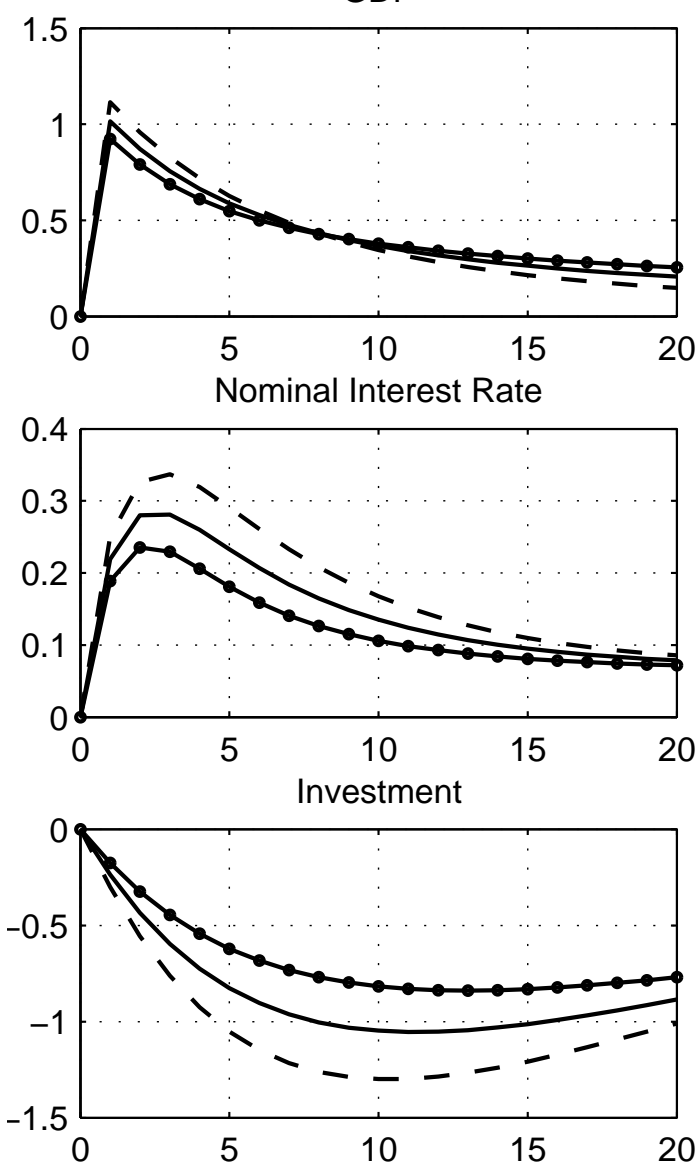

Inflation
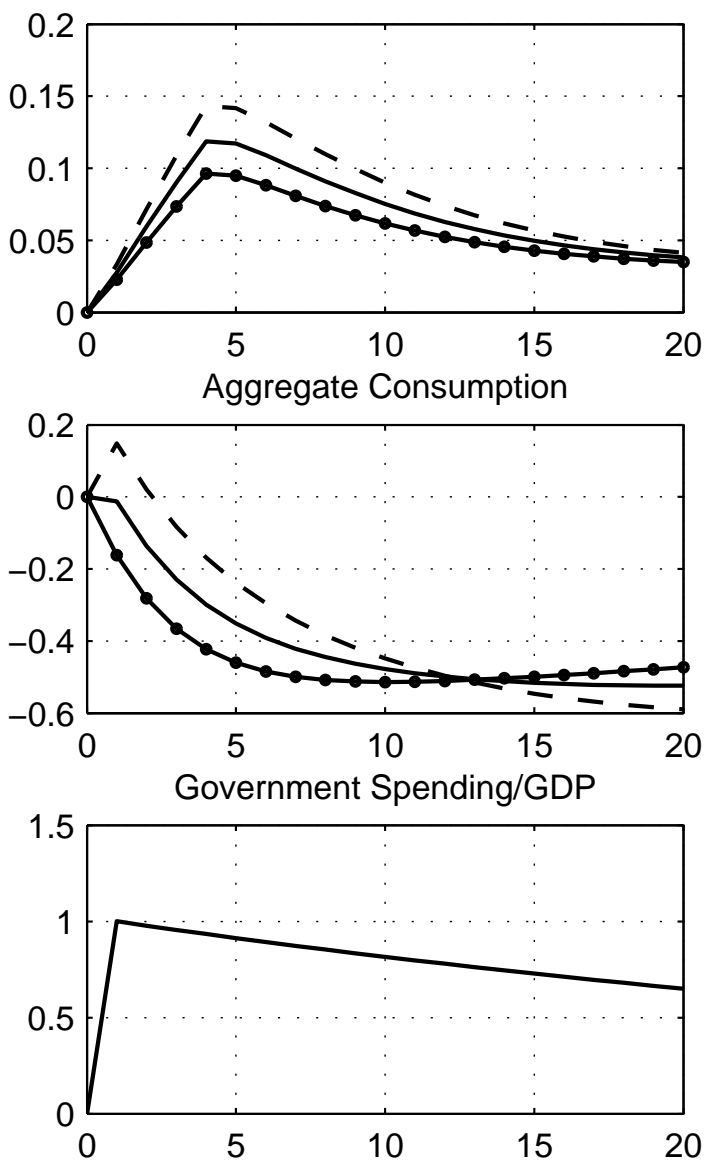

Notes: Horizontal axis represents quarters after the shock. Units of the vertical axis are percentage deviations from steady-state values except government spending. Government spending is expressed as a share of GDP in percentage point deviations from the respective steady-state ratio. Inflation is the rate of inflation over the previous four quarters. Nominal interest rate is annualized. Other variables are expressed in quarterly terms. $\omega$ refers to the population share of rule-of-thumb households. The value of $\omega=0.265$ (US_CCTW 10) corresponds to the posterior mean estimate of Cogan et al. (2010). The simulation is carried out under monetary policy rule of Cogan et al. (2010).

ing the same data as Smets and Wouters (2007), Cogan et al. (2010) estimate the share of Keynesian rule-of-thumb consumers in the population, $\omega$, jointly with the other structural parameters of the model. For the Bayesian estimation, the prior mean is assumed to be $50 \%$. The resulting posterior mean is 27 percent with a standard deviation of 6 percent. Meanwhile, the other models used in Coenen et al. (2012) calibrate or estimate the population share of financially constrained households to values between 20 percent and 50 percent.

Figure 7 reports on the effects of the fiscal policy shock for three different values of the share of rule-of-thumb consumers that is denoted by $\omega$ in the US_CCTW10 model. There are 6 panels displaying simulation outcomes for GDP, inflation, the nominal interest rate, consumption, invest- 
ment and government purchases. Each panel contains three lines indicating outcomes with a share of rule-of-thumb households of 0 percent $(\omega=0), 26.5$ percent $(\omega=0.265$, US_CCTW10) and 50 percent $(\omega=0.5){ }^{5}$ For each simulation, the other parameters are kept unchanged at the posterior means estimated by Cogan et al. (2010). Noteworthy, setting $\omega=0$ or $\omega=0.5$ implies a deviation from the point estimate delivering the optimal fit of the model to the data. The simulation outcomes are best understood as a sensitivity exercise with respect to the single parameter $\omega$.

With $\omega=0$ there are no rule-of-thumb households. All consumers are forward-looking and base their decision on expected life-time income as in Smets and Wouters (2007). By contrast, the value of 50 percent can be considered an upper limit of estimates for the share of rule-of-thumb consumers found in the literature on the U.S. economy. In all three simulations, government spending increases on impact by 1 percent of GDP and gradually returns to the steady-state ratio of government spending to output (lower right panel).

The effect on GDP increases with the population share of rule-of-thumb consumers. Yet, the quantitative differences in the GDP impact of the fiscal shock are not very large. The reason is that a crowding-out effect becomes more pronounced with a larger share of rule-of-thumb consumers. On the one side, aggregate consumption is higher with higher values of $\omega$. With $50 \%$ of rule-ofthumb consumers, aggregate consumption even increases a bit in the first quarter consistent with the Keynesian multiplier effect. However, in response to higher GDP and higher inflation, the central bank raises the nominal interest rate. As prices adjust sluggishly due to nominal rigidities, the real interest rate (not shown) rises as well and by a larger amount for higher values of $\omega$. Higher real rates reduce demand for investment purposes and incentivize forward-looking households to postpone consumption. Thus, the expansion in government spending crowds out private spending on investment and consumption. The model with rule-of-thumb consumers also accounts for the dynamics of government debt and taxes. First, government debt increases, then lump-sum taxes respond so as to return debt to the initial debt-to-GDP ratio. While rule-of-thumb consumers ignore the reduction of future disposable income, forward-looking consumers respond by reducing current consumption.

\section{Parameter sensitivity analysis: Central bank reaction function}

Next, the effect of monetary accommodation is easily evaluated by changing the response coefficients in the monetary policy rule. This can either be accomplished by picking different pre-set rules under the "One model, many policy rules" menu or by entering different coefficients in the sub-menu for the "User-specified rule". We compare the outcomes under the model-specific estimated rule from Cogan et al. (2010) (CCTW10 Rule) with the model-specific rule from Bernanke et al. (1999) (BGG99 rule). The latter rule will also be used in Section 6 when we compare the

\footnotetext{
${ }^{5}$ Technically, users can easily change this structural parameter by editing the model file US_CCTW10.mod in the subdirectory /MODELS/US_CCTW10 of the archive. The parameter is found under "// fixed parameters " and denoted by " omega $=0.2651$; // share of rule-of-thumb consumers" as in the published article. Then, the user simply needs to run the fiscal shock simulation in the menu "One model, many policy rules", repeating it every time he has edited the model file. Three sets of results can be saved in Excel files and then displayed in graphs.
} 
Figure 8: IMPULSE RESPONSES TO AN EXPANSIONARY FISCAL POLICY SHOCK IN THE US_CCTW10 MODEL WITH ALTERNATIVE MONETARY POLICY RULES

BGG99 Rule
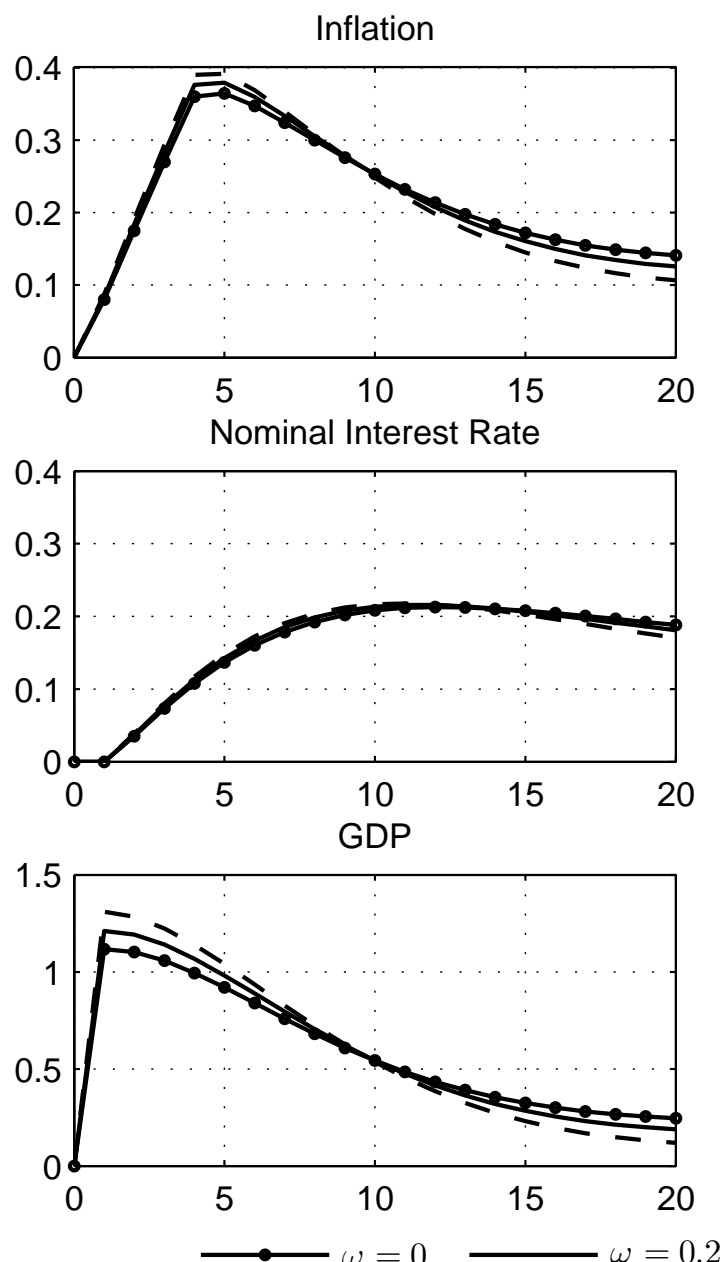

CCTW10 Rule
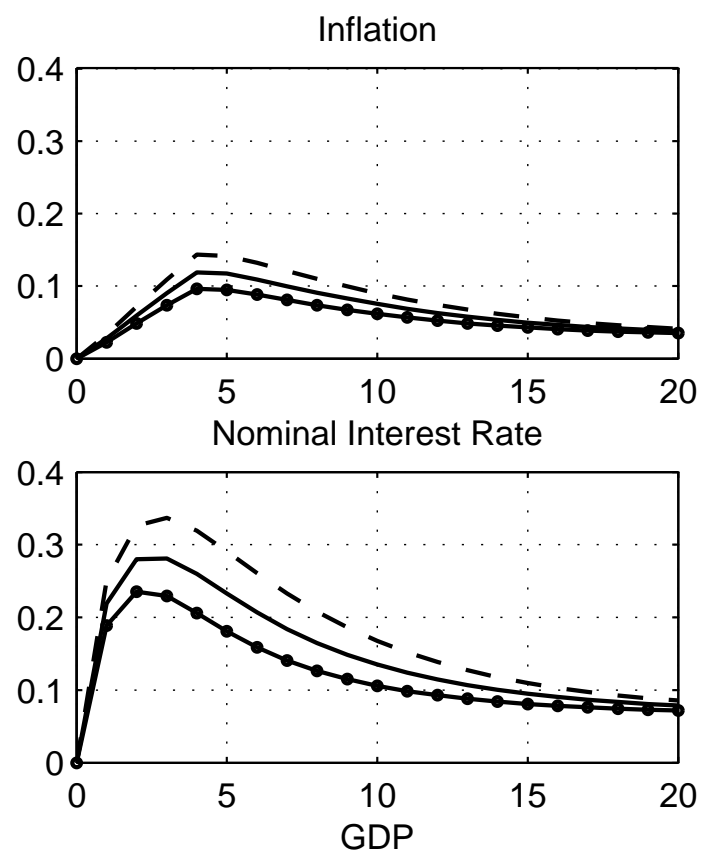

Notes: Horizontal axis represents quarters after the shock. Units of the vertical axis are percentage deviations from steady-state values. Inflation is the rate of inflation over the previous four quarters. Nominal interest rate is annualized. GDP is expressed in quarterly terms. BGG99 rule refers to monetary policy rule of the NK_BGG99 model by Bernanke et al. (1999). CCTW10 rule refers to the estimated rule of the US_CCTW10 model by Cogan et al. (2010).

small New Keynesian model of Bernanke et al. (1999) (NK_BGG99 model) to more recent macrofinancial models. Here, the BGG99 rule is of interest because it responds only to lagged values for inflation and the interest rate and does not react to GDP (see equation 126 Thus, it should be much more accommodative than the CCTW10 rule.

Figure 8 presents the implications of the government spending shock for inflation, nominal

\footnotetext{
${ }^{6}$ This rule can be specified using the "User-specified rule" tab in the panel Monetary Policy Rules in the MMB graphical user interface. More specifically, the user needs to assign ' 0.9 ' for the entry for interest ( $t-1)$ and ' 0.11 ' for the entry for inflationq $(t-1)$.
} 
interest rate and output under the two different policy rules. The panels in the right column report selected results from the previous exercise with CCTW10 rule (compare Figure 7). Again, we consider the same three values for $\omega$. The panels in the left column display the outcomes simulated under the BGG99 rule.

The increase in government purchases induces much stronger effects on aggregate GDP under the BGG99 rule. Even in the absence of rule-of-thumb consumers, $(\omega=0)$, the GDP impact exceed unity in the first 4 quarters. The much more accommodative monetary policy regime exhibited by the BGG99 rule allows for a Keynesian multiplier effect. Private consumption rises due to higher government consumption. This crowding-in effect outweighs the negative wealth effect coming from higher anticipated future taxes. The comparison emphasizes the importance of fiscal-monetary interactions for the effects of discretionary fiscal policy.

\subsection{Monetary policy transmission: Comparing generations of models}

The Macroeconomic Model Data Base serves as an archive of models and contains models developed at different times and based on different theories about how the economy functions. Thus, it offers the possibility to compare different generations of models and their policy implications. One would expect that policy implications change substantially over time, either because new theories offer new insights in macroeconomic interdependencies or because new estimation methods and new data induce different estimates of key parameters. But there may also be surprising similarities.

For example, Taylor and Wieland (2012) compare four different models of the U.S. economy that were developed and estimated at different times with different data, and find very similar estimates of the transmission of a monetary shock to GDP. This holds at least when a common central bank reaction function is used. Here we extend this comparison to a fifth model that was estimated very recently. The models are listed in Table 5. MMB users can easily replicate and extend this comparison further with the MMB graphical user interface "One policy rule, many models".

Table 5: THREE MODEL GENERATIONS

\begin{tabular}{ll}
\hline Notation & Description \\
\hline G7_TAY93 & $\begin{array}{l}\text { Taylor (1993b): 1st generation New Keynesian model with rational ex- } \\
\text { pectations, wage and price rigidities }\end{array}$ \\
\cline { 2 - 2 } US_ACELm & $\begin{array}{l}\text { Christiano et al. } \\
\text { Dynamic Stochastic General Equilibrium model }\end{array}$ \\
US_SW07 & Smets and Wouters (2007): 2nd generation NK-DSGE model \\
US_DG08 & $\begin{array}{l}\text { De Graeve (2008): 3rd generation NK-DSGE model with financial } \\
\text { frictions }\end{array}$ \\
US_CMR14 & Christiano et al. (2014): 3rd generation NK-DSGE model, financial \\
& frictions \\
\hline
\end{tabular}

The models 
The G7_TAY93 model, which is a multicountry model of the G7 economies built more than 20 years ago, has been used extensively in the model comparison projects of the late 1980s and 1990s (see Section 2.2. It has New Keynesian properties such as nominal wage and price rigidities, rational expectations and policy rules. However, it does not yet incorporate the complete set of microeconomic foundations developed in the real and monetary business cycle literature. We refer to it as a first-generation New Keynesian model.

US_ACELm77 and US_SW07 are the best-known representatives of the second generation of empirically estimated New Keynesian models with additional microeconomic foundations, often referred to New Keynesian DSGE models. Although they differ from G7_TAY93 also in terms of the estimation approach, data and sample span, they exhibit almost identical GDP effects of an unexpected change in the federal funds rate. Following the global financial crisis, New Keynesian DSGE models have been fitted out with more detailed financial sectors and financial frictions that serve to amplify financial and economic shocks. Taylor and Wieland (2012) showed that one of these third-generation New Keynesian models, the US_DG08 model, also indicated similar monetary policy effects.

Here, we extend the comparison exercise by bringing one more model with financial frictions into the picture. Noteworthy, the US_CMR14 model is the only one among these five models estimated on data that covers the Great Recession (the sample spans 1985:Q1 to 2010:Q2) and includes financial time series such as credit to non-financial firms, the slope of the term structure, credit spreads on corporate bonds, and an index of stock prices.

US_CMR14 Model Description: Christiano et al. (2014) introduce the financial accelerator mechanism of Bernanke et al. (1999) into an otherwise standard New-Keynesian model, such as the model of Christiano et al. (2005). This mechanism is described in more detail in Section 6. In contrast to earlier models with financial frictions (see, e.g., Christensen and Dib (2008), De Graeve (2008)), the authors introduce a shock to the variance of idiosyncratic productivity that influences individual entrepreneur's return to capital. It is referred to as a risk shock. With an agency problem between entrepreneurs and banks, a positive risk shock increases the required return on borrowing, that is, the external finance premium. As a consequence, entrepreneurs' borrowing is reduced and investment declines. As capital prices fall, entrepreneurial net worth decreases, which in turn raises the external finance premium further. These amplification effects are propagated to the real economy over time. Importantly, the authors' analysis suggests the risk shock is a major driving force in the U.S. business cycles.

\footnotetext{
${ }^{7}$ As the impulse response functions for the monetary policy shock in Altig et al. (2005) are almost identical to those of Christiano et al. (2005). Altig et al. (2005), however, incorporate two additional shocks (a neutral and investmentspecific technology shock). The Macroeconomic Model Data Base includes the model of Altig et al. (2005).
} 


\section{Strikingly similar impulse responses to a monetary policy shock}

Figure 9 displays the effects of a one-percentage-point unexpected increase in the federal funds rate on output, inflation, and the interest rate itself in all five models under two alternative monetary policy rules. The panels on the left side show the outcomes when the interest rate is set according to the monetary policy rule estimated in Smets and Wouters (2007) (SW rule), while the panels on the right side refer to the outcomes under the monetary policy rule estimated in Christiano et al. (2014) (CMR rule). The SW rul $\mathrm{g}^{8}$ and the CMR rule are given by equations 10 ) and 111 , respectively.

$$
\begin{aligned}
& i_{t}^{z}=0.81 i_{t-1}^{z}+0.39 p_{t}^{z}+0.97 q_{t}^{z}-0.90 q_{t-1}^{z}+\eta_{t}^{i} . \\
& i_{t}^{z}=0.85 i_{t-1}^{z}+0.36 p_{t}^{z}+0.05 y_{t}^{z}-0.05 y_{t-1}^{z}+\eta_{t}^{i} .
\end{aligned}
$$

The superscript $z$ refers to common variables, that are defined consistently and therefore allow quantitative comparisons. The monetary policy instrument is the annualized short-term federal funds rate in quarter $t$ denoted by $i_{t}^{z}$. $p_{t}^{z}$ refers to the annualized quarter-to-quarter rate of inflation, $y_{t}^{z}$ is the deviation of quarterly real GDP from its long-run potential, while $q_{t}^{z}$ refers to the output gap defined as the difference between actual GDP and the level of GDP that would be realized if prices and wages were flexible. All variables are expressed in percentage deviations from steadystate values. $\eta_{t}^{i}$ refers to the common monetary policy shock.

Under the SW rule, US_SW07, US_ACELm and G7_TAY93 indicate almost identical GDP responses and quite similar inflation responses to the interest rate shock. GDP declines by 25 to 30 basis points within 3 to 4 quarters and then returns to its steady-state level again. The effects are only slightly larger in the US_DG08 model that was also considered by Taylor and Wieland (2012).

Interestingly, the maximum GDP effect in the US_CMR14 model is again of the same magnitude, about 30 basis points within 4 quarters under the SW rule. Yet, it is much more persistent. GDP returns very slowly to steady state. It barely moves back over the first 20 quarters. It seems monetary policy has become more powerful in terms of inducing lasting consequences for the real side of the economy. Clearly, this finding requires further study.

When using the CMR rule, we obtain greater effects of the policy shock on GDP and inflation in all five models. The reason is that CMR rule is more acommodative. Its reaction coefficients concerning real GDP are smaller. Yet again, US_SW07, US_ACELm and G7_TAY93 imply very similar GDP effects, on the scale of a reduction of 45 to 50 basis points within 3 to 4 quarters. With this rule, the differences in the third generation of New Keynesian models with financial frictions come out more clearly. In US_DG08 the impact on GDP is quite a bit stronger reaching -90 basis points, while it is again much longer-lasting in US_CMR14.

As a further check on the source of the stronger, more lasting effect of monetary policy on real

\footnotetext{
${ }^{8}$ The monetary policy shock in the estimation of Smets and Wouters (2007) exhibits weak serial correlation with a correlation coefficient of 0.15 . In MMB the policy shocks are iid.
} 
Figure 9: Impulse Responses to a Contractionary Monetary Policy Shock in SeLeCTEd Models of THE U.S. ECONOMy Under Alternative Policy Rules

SW Rule
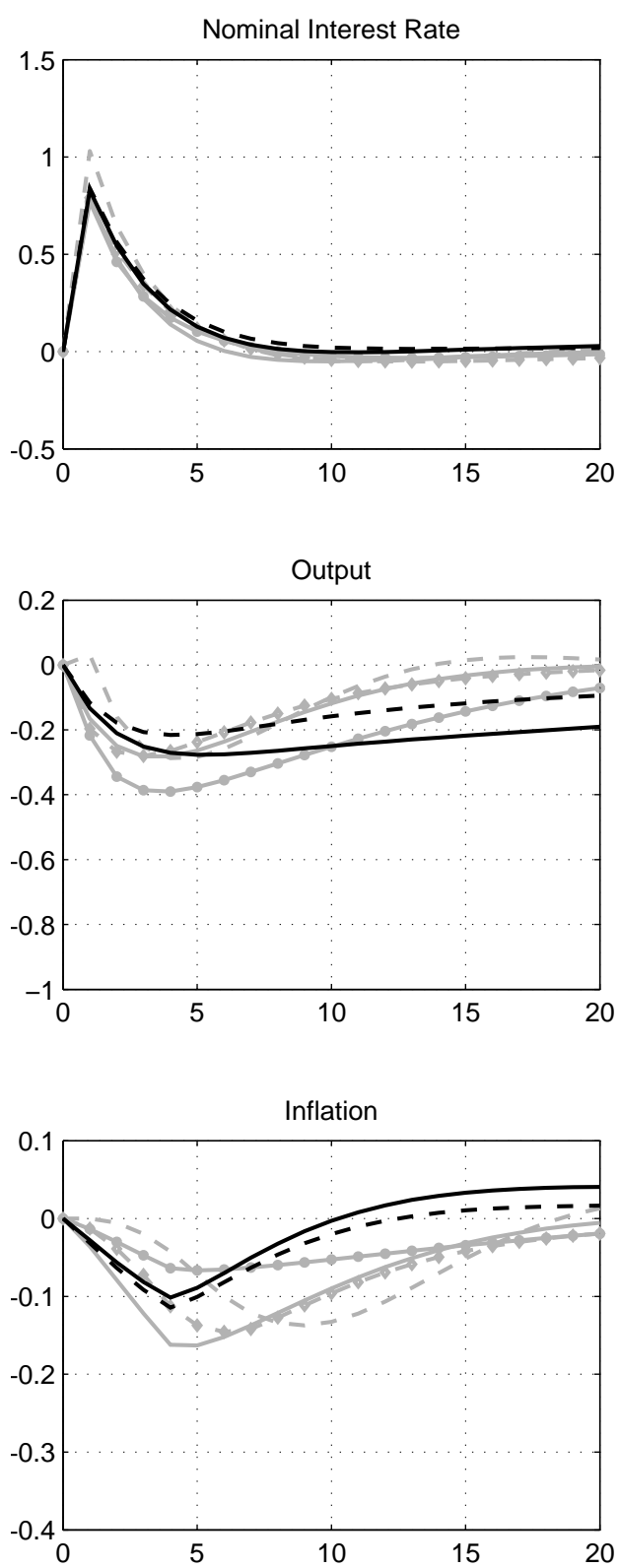

US_SW07

-- - US_ACELm
CMR Rule
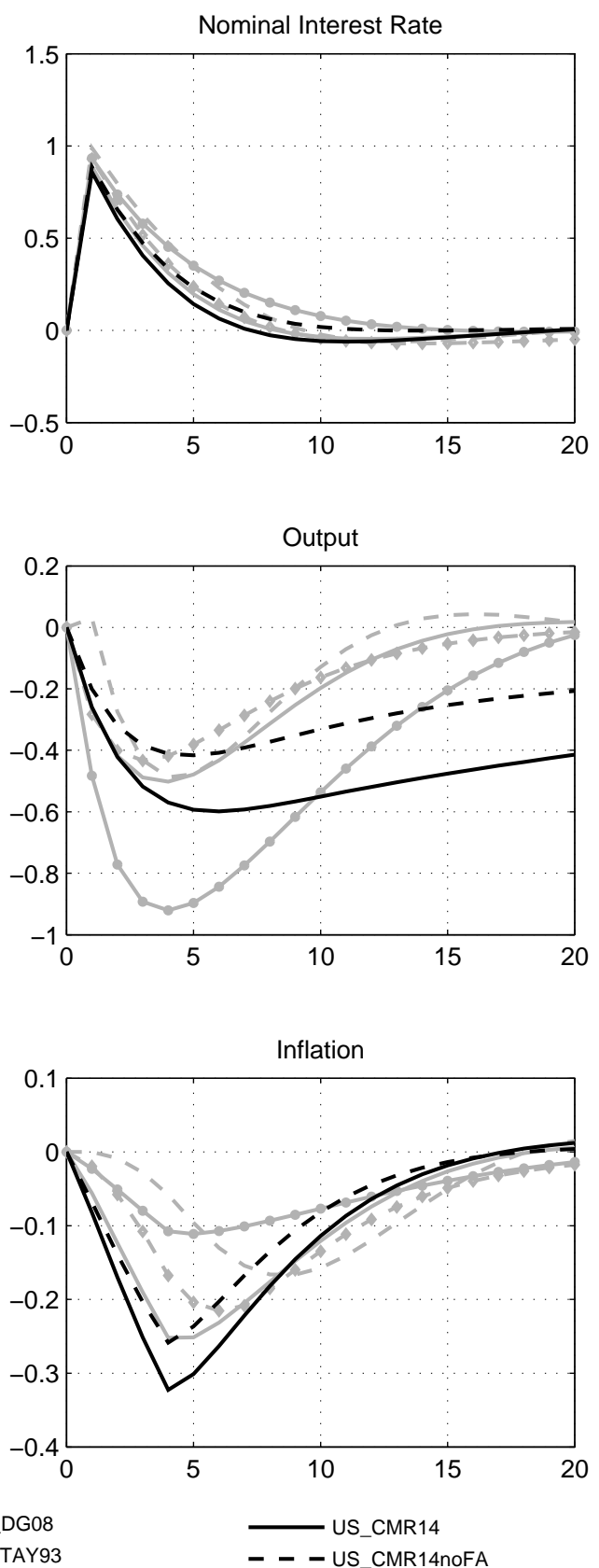

Notes: Horizontal axis represents quarters after the shock. Units of the vertical axis are percentage deviations from steady-state values. Inflation is the rate of inflation over the previous four quarters. Nominal interest rate is annualized. Output is expressed in quarterly terms. SW rule refers to the monetary policy rule of Smets and Wouters (2007). CMR rule refers to the monetary policy rule of Christiano et al. (2014). US_SW07 is the model of Smets and Wouters (2007); US_ACELm replicates the model of Christiano et al. (2005); G7_TAY93 is the model of Taylor(1993b); US_CMR14 is the model of Christiano et al. (2014); US_CMR14noFA is the modified version of US_CMR14 without the financial friction. 
GDP, we modify the US_CMR14 in order to shut down the financial accelerator mechanism. We label this modified version of the model US_CMR14noFA. All other parameters are kept at the values in the original model specification. Noteworthy, the US_CMR14noFA model is structurally very close to the US_ACELm model, the main difference being the presence of the cost channel in US_ACELm. We find that the GDP response is less pronounced in the version without the financial accelerator, yet it remains somewhat more persistent than in the other models.

Unusually persistent real effects of monetary shocks in the model of Christiano et al. (2014)

To investigate the possible origin of the unusually long-lasting real effects of monetary policy in the US_CMR14 model, we repeat the same exercise with four different versions of the model. In doing so, we always use the model-specific rule, i.e. the CMR rule. In addition to US_CMR14noFA, which shuts down the financial accelerator, we consider a version that shuts down nominal wage rigidities (US_CMR14noNW), and a version without both, wage rigidities and financial frictions (US_CMR14noFA\&NW). Figure 10 presents the resulting impulse responses.

The persistent response of GDP in the baseline model (US_CMR14) is reflected in both investment and consumption. Investment falls for eight quarters and then returns very slowly to the steady state. Consumption falls and stays far below the steady state for about thirty quarters and then starts returning to the steady state. Such a long-lasting effect of a monetary shock on household consumption in real terms appears rather unrealistic. In particular, as inflation, nominal and real interest rates return to steady state in 10 quarters.

While the magnitude of the effect is reduced in the model without the financial accelerator (US_CMR14noFA), consumption and investment remain highly persistent. In the model without nominal wage rigidities (US_CMR14noNW), real wages fall sharply, while hours worked decline somewhat. The reason is that intermediate good producing firms respond to the contractionary policy shock by adjusting prices rather than quantities. It leads to smaller effects on output and larger effects on inflation. However, the dynamics of output, consumption and investment are still very persistent. For instance, consumption first returns to the steady state ten quarters after the shock but then continues to decrease even more than during the initial 10 quarters.

Finally, in the model without wage rigidities and financial friction (US_CMR14noFA\&NW), the real effects of a policy shock are much reduced. The magnitude of the maximum output effect is about one-third of that in the baseline model. More importantly, the strikingly persistent dynamic responses of real variables disappear. Output, investment, consumption and hours worked return close to steady state in 10 to 15 quarters.

Our comparative exercise shows that the US_CMR14 model implies highly persistent output effects of monetary policy shocks relative to other estimated models of the U.S. economy. Given that the policy debate after the global financial crisis and the Great Recession has been dominated by the fear that monetary policy has become less effective, this result is surprising. The comparisons with modified versions of the model suggests that this feature of the US_CMR14 model is 
Figure 10: Contractionary Monetary Policy Shock in MOdified Versions of The CHRISTIANO ET AL. (2014) MODEL WITH CMR RULE
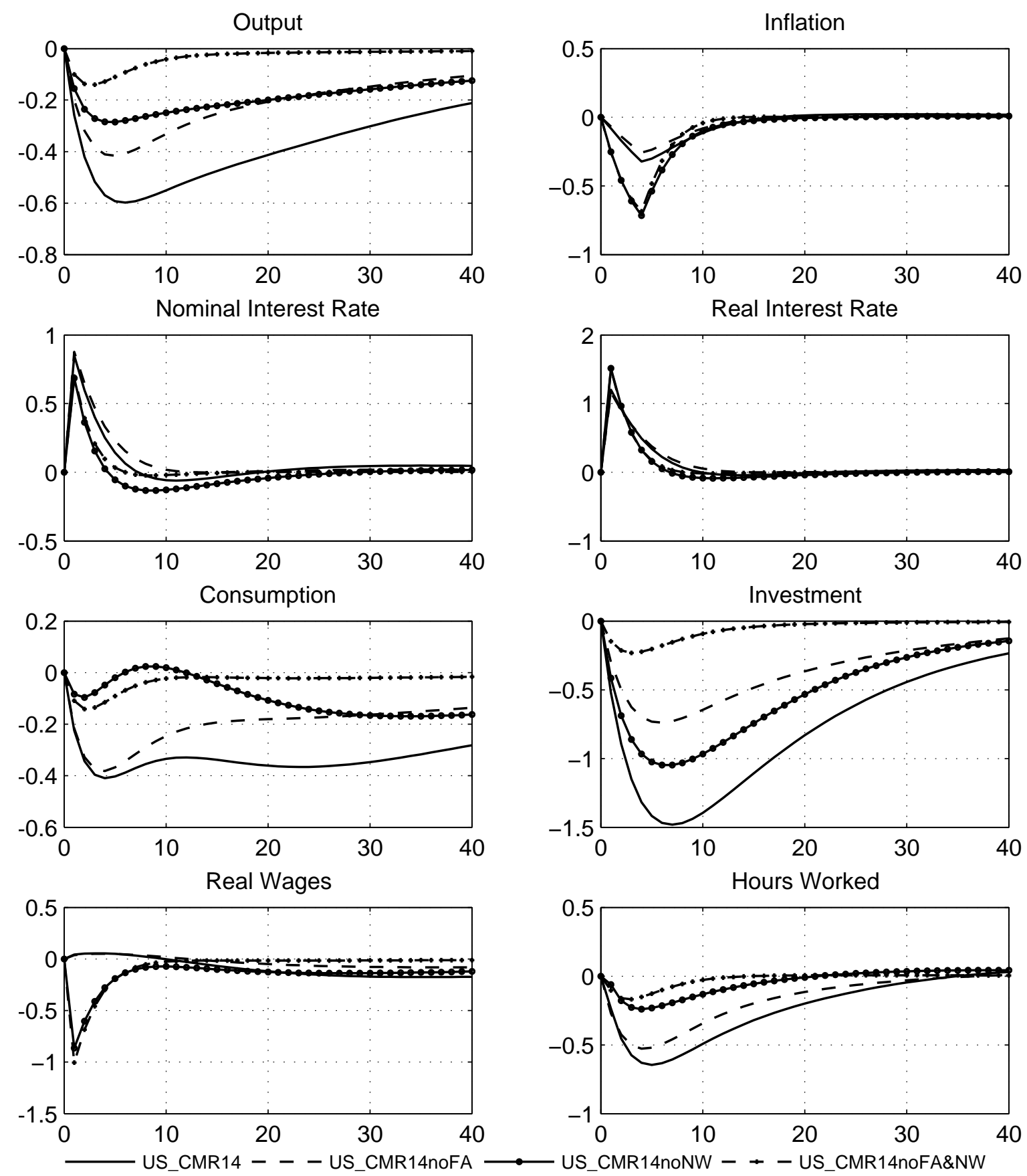

Notes: Horizontal axis represents quarters after the shock. Units of the vertical axis are percentage deviations from steady-state values. Inflation is the rate of inflation over the previous four quarters. Nominal interest rate is annualized. Other variables are expressed in quarterly terms. US_CMR14 is the model of Christiano et al.(2014); US_CMR14noFA is the version of US_CMR14 without the financial friction; US_CMR14noNW is the version of US_CMR14 without nominal wage rigidities; US_CMR14noFA\&NW is the version of US_CMR14 without both the financial friction and nominal wage rigidities. 
rooted in the parameter estimates that govern the importance of wage rigidities and of the financial accelerator. Yet, it would be important to explore further whether this effect depends on unusual combinations of parameter estimates and whether the extreme persistence disappears if the model is estimated over part of the data sample.

\subsection{Predicted effects of identified policy shocks: United States versus euro area}

When a model comparison should make use of model-specific policy rules

In the preceding exercise, we have considered the consequences of monetary policy shock across models when the central bank in each model applies the same common policy rule. The idea of these simulations is to examine model differences stemming from model structure, while eliminating the differences stemming from a model-specific monetary policy rule. They correspond exactly to the approach laid out in Section 3 Assuming a common rule serves its purpose in making a clean comparison of the policy implications of different model structures.

However, there are other questions that can be answered with model comparisons that employ model-specific rules. For example, if one wants to compare the forecasting performance of different models, the model should be used as fitted to the data. Using a different policy rule would reduce the fit of the model to the data it was estimated on, and its forecasting performance would presumably deteriorate. Thus, for such comparison each model should be used with the model-specific policy rules that were estimated along with the rest of the model.

Hence any question that involves comparing model fit would make use of model-specific rules. The question to be considered here concerns the empirical degree of model uncertainty about the consequences of identified monetary policy shocks in the United States versus the euro area. Specifically, we aim to assess the range of predicted effects across models. Conditional on the structural assumptions of a model and the sample the model was estimated on, the impulse response under the model-specific rule represents the most likely data-driven reaction of the economy to the monetary policy shock. The comparison exercise then provides a measure of the degree of model uncertainty about monetary policy transmission.

\section{Models with different structural features estimated with U.S. and euro area data}

Specifically, we choose models from the MMB archive for which all equations are jointly estimated and the model-specific monetary policy rule is formulated for the nominal short-term interest rate. This selection includes twelve U.S. models and eight euro area models (see Table 6). Although all models share certain New Keynesian features, there is a lot of heterogeneity in terms of structural assumptions, observables and estimation techniques?

All the U.S. models and most euro area models are closed economies. Exceptions include EA_SR07 and EA_QUEST3, which are small open economies, and the two-country models EAES_RA09 and EA_QR14. Most models only consider forward-looking permanent-income households. EA_QUEST3

\footnotetext{
${ }^{9} \mathrm{~A}$ brief description of each model is included in the MMB software package and can be downloaded from the MMB website.
} 
Table 6: ESTIMATE MODELS USED IN THE COMPARISON ACROSS ECONOMIES

\begin{tabular}{|c|c|c|c|}
\hline \multicolumn{2}{|c|}{ Estimated U.S. Models } & \multicolumn{2}{|c|}{ Estimated euro area Models } \\
\hline US_ACELm & Christiano et al. (2005) & EA_SW03 & Smets and Wouters (2003) \\
\hline US_IAC05 & Iacoviello (2005) & EA_SR07 & Adolfson et al. (2007) \\
\hline US_MR07 & Mankiw and Reis (2007) & EA_QUEST3 & Ratto et al. $(2009)$ \\
\hline US_RA07 & Rabanal (2007) & EAES_RA09 & Rabanal (2009) \\
\hline US_SW07 & Smets and Wouters (2007) & EA_CKL09 & Christoffel et al. (2009) \\
\hline US_CD08 & Christensen and Dib (2008) & EA_GE10 & Gelain (2010) \\
\hline US_DG08 & De Graeve (2008) & EA_GNSS10 & Gerali et al.(2010) \\
\hline US_PM08fl & Carabenciov et al. (2008) & EA_QR14 & Quint and Rabanal (2014) \\
\hline US_IN10 & Iacoviello and Neri (2010) & & \\
\hline US_CCTW10 & Cogan et al. $(2010)$ & & \\
\hline US_IR11 & Ireland $(2011)$ & & \\
\hline US_CMR14 & Christiano et al. (2014) & & \\
\hline
\end{tabular}

Note: The first and third columns contain the model name in the MMB for the respective paper.

and US_CCTW10, however, also include rule-of-thumb households. Models with housing finance, such as US_IAC05, US_IN10, EA_GNSS10, and EA_QR14 feature two types of households that behave as borrowers and savers, respectively. The difference in decision making arises from differences in their discount factors. Savers are more patient than borrowers. Impatient agents face a borrowing constraint and use housing as collateral for borrowing.

Another financial friction that influences credit demand-the financial accelerator mechanism of Bernanke et al. (1999) - is incorporated in the US_CD08, US_DG08, US_CMR14, and EA_GE10 models. Frictions in credit supply are considered in the EA_GNSS10 model, which includes a more detailed banking sector. US_PM08fl, the IMF's small projection model for the U.S. economy, also includes a macro-financial linkage in form of a behavioral relation between bank lending conditions and the real economy. The propagation mechanisms generated by financial frictions are to be studied more thoroughly in Section 6

The models considered in this exercise also incorporate different labor market structures. Some models (US_IAC05, US_CD08, US_IR11, EA_QR14) assume competitive labor markets, but a majority of the models accounts for monopolistic competition in labor supply and Calvo-style rigidity in nominal wages. EA_CKL09 additionally introduce Mortensen and Pissarides (1994) type of matching frictions in the labor market.

Furthermore, US_MR07 differs from all other models due to the assumption of sticky information. In this model, only a fraction of agents (consumers, workers and firms) updates their information regularly when making decisions. The other agents are inattentive. This feature gives rise to sluggish macroeconomic adjustment.

With regard to model-specific interest rate rules, most models feature interest rate smoothing as well as a reaction to inflation and a real variable (typically, the output gap or output growth). The 
exceptions are US_CD08 and EA_SR07, where the monetary policy rule also includes reactions to money growth and the real exchange rate, respectively.

Finally, there are also important differences in terms of the time series employed in estimating the models. At a minimum, these include real GDP, inflation and the short-term nominal interest rate. Most of the models, however, are estimated on a larger set of observables. For example, Smets and Wouters (2007), De Graeve (2008), Smets and Wouters (2003) and Gelain (2010) use seven macroeconomic time series: real GDP, inflation, consumption, investment, real wages, employment and the short-term nominal interest rate. Adolfson et al. (2007) employ fifteen macroeconomic time series to estimate the euro area model of Sveriges Riksbank (EA_SR07). Iacoviello and Neri (2010) use ten observables, including measures of housing construction and prices. In terms of sample period, the U.S. models are typically estimated on longer samples than the euro area models. Most models are estimated with Bayesian techniques. However, US_CD08 and US_IR11 are estimated with maximum likelihood techniques, while US_ACELm and US_IAC05 are estimated by minimizing the distance between VAR-based and model-implied impulse responses.

\section{Dynamic responses of output, inflation and interest rates: U.S. vs euro area}

Figure 11 reports the outcomes for a one-percentage-point contractionary shock to the nominal interest rate under model-specific rules 10 The panels in the left column display the results for twelve estimated models of the U.S. economy, while the panels in the right column show the results for eight euro area models.

In every case, the unexpected increase in the nominal interest rate leads to a decline in output and inflation. Due to sticky prices, the real interest rate rises, which depresses aggregate demand. Lower demand curbs production. As a fraction of price setters adjust to lower demand, inflation falls.

At first glance, there appears to be considerable variation in the magnitude and dynamic patterns of effects. Yet, this impression results from a few outliers. Outliers with regard to the output are US_IAC05, US_RA07 and EA_SW03, while US_MR07, US_RA07 and EA_SW03 are outliers with regard to inflation dynamics. Except for US_IAC05, the strong reactions to the policy shock are largely due to a coefficient near unity on the lagged interest rate in the policy rule ${ }^{11}$ The anticipation of a longer period of higher interest rates induces a larger and longer lasting effect on output and inflation, because households and firms take into account expectations of future interest rates in their decision making. In the case of US_IAC05, the lack of important real rigidities, such

\footnotetext{
${ }^{10} \mathrm{We}$ obtain simulation results in two ways. When a model-specific policy rule is nested in the generalized rule in MMB, it is available for simulation in each model using the options menu One model, many policy rules. If this is not the case, we use the replication files for the original models, which are provided together with the MMB comparison software.

${ }^{11}$ The coefficients on the lagged interest rate in the policy rule of the models with the strongest responses are as follows: 0.94 for US_RA07, 0.92 for US_MR07, 0.96 for EA_SW03. Noteworthy, the model-specific policy rule of Mankiw and Reis (2007) does not explicitly include a lagged interest rate but the policy shock is modeled as an AR(1)-process with the persistence coefficient of 0.92 .
} 
Figure 11: Impulse Responses to a Contractionary Monetary Policy Shock in VARIOUS MODELS WITH MODEL-SPECIFIC RULES

United States
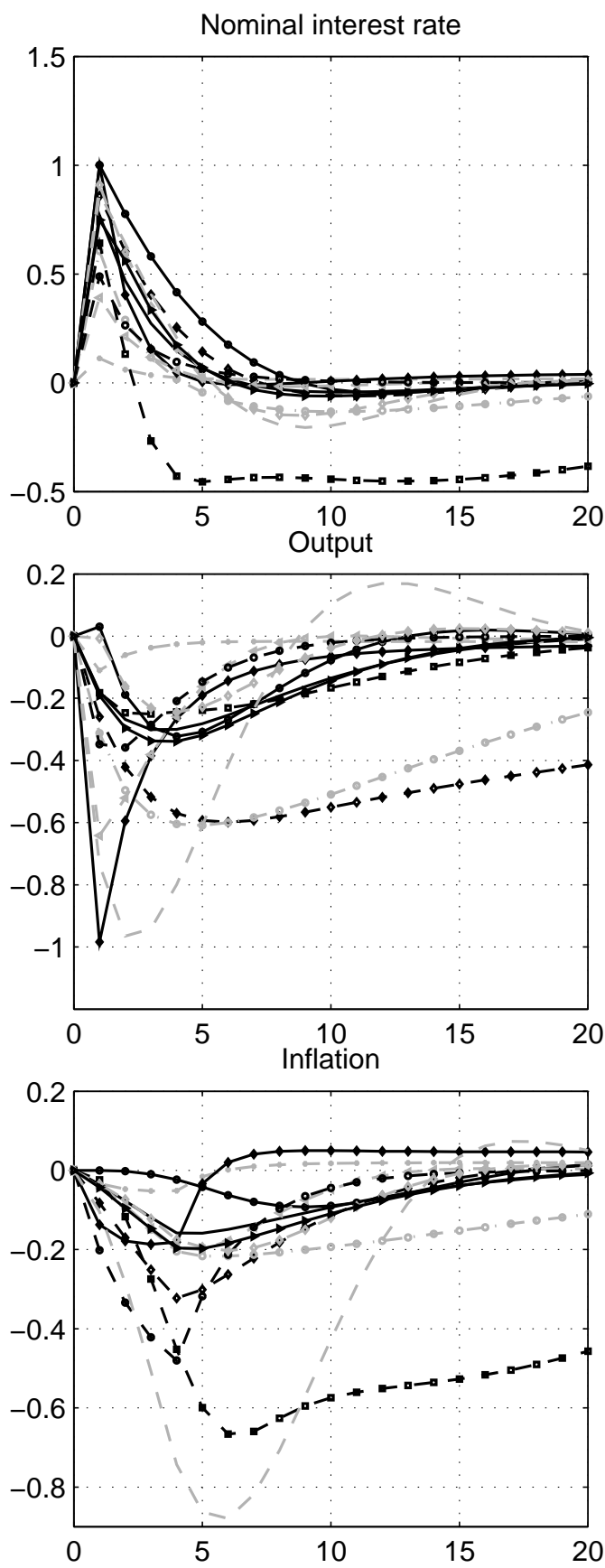

Euro Area
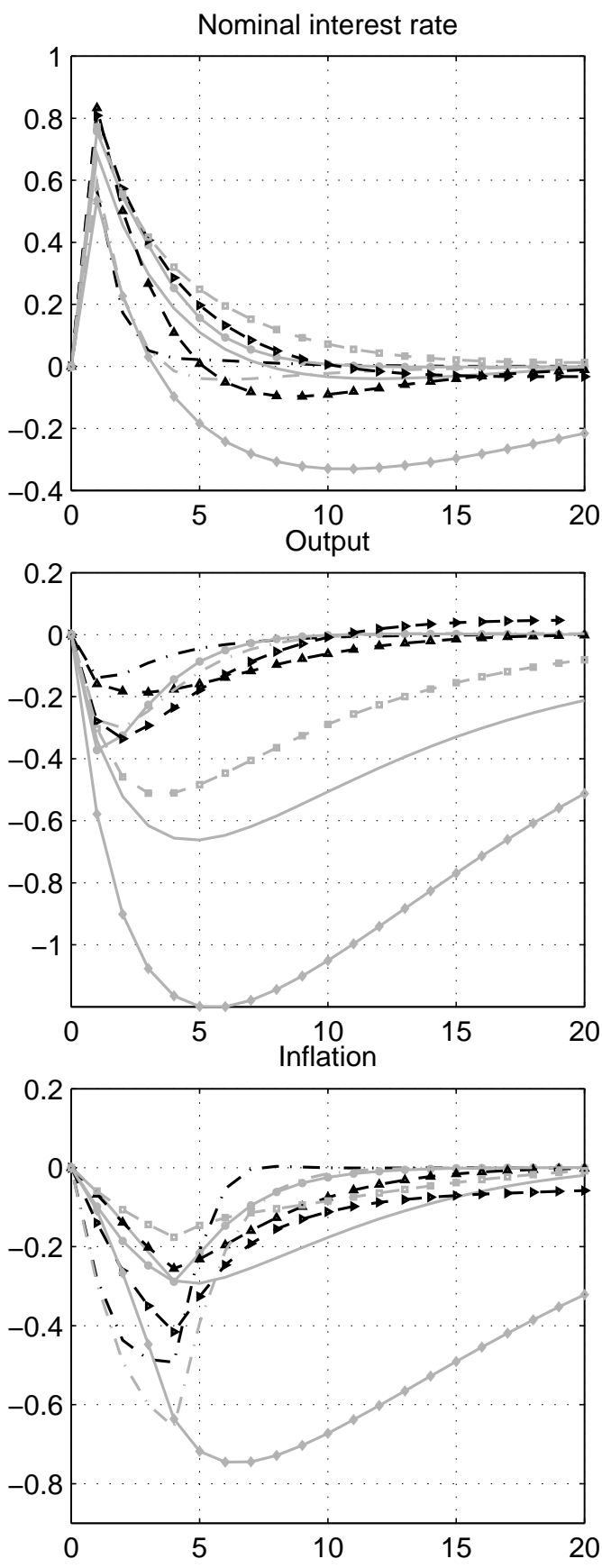

Notes: Horizontal axis represents quarters after the shock. Units of the vertical axis are percentage deviations from steady-state values. Inflation is the rate of inflation over the previous four quarters. Output is expressed in quarterly terms. 
Table 7: EFFECTS OF A ONE PERCENTAGE POINT UNEXPECTED INCREASE IN THE POLICY RATE ON OUTPUT AND INFLATION IN THE U.S. AND THE EURO AREA MODELS

\begin{tabular}{|c|c|c|c|c|}
\hline & \multicolumn{2}{|c|}{ Output } & \multicolumn{2}{|c|}{ Inflation } \\
\hline & Timing & Magnitude & Timing & Magnitude \\
\hline \multicolumn{5}{|c|}{ (a) Estimated U.S. Models } \\
\hline US_ACELm & 4 & $-0.32 \%$ & 9 & $-0.09 \%$ \\
\hline US_IAC05 & 1 & $-0.98 \%$ & 3 & $-0.19 \%$ \\
\hline US_MR07 & 3 & $-0.25 \%$ & 6 & $-0.67 \%$ \\
\hline US_RA07 & 2 & $-0.96 \%$ & 6 & $-0.88 \%$ \\
\hline US_SW07 & 4 & $-0.34 \%$ & 5 & $-0.20 \%$ \\
\hline US_CD08 & 1 & $-0.11 \%$ & 3 & $-0.05 \%$ \\
\hline US_DG08 & 5 & $-0.61 \%$ & 6 & $-0.22 \%$ \\
\hline US_PM08fl & 4 & $-0.25 \%$ & 6 & $-0.20 \%$ \\
\hline US_IN10 & 1 & $-0.64 \%$ & 5 & $-0.20 \%$ \\
\hline US_CCTW10 & 3 & $-0.30 \%$ & 5 & $-0.16 \%$ \\
\hline US_IR11 & 2 & $-0.36 \%$ & 4 & $-0.48 \%$ \\
\hline US_CMR14 & 6 & $-0.60 \%$ & 4 & $-0.32 \%$ \\
\hline Model averages & 3.0 & $-0.48 \%$ & 5.2 & $-0.30 \%$ \\
\hline Standard deviations & 1.7 & $0.28 \% \mathrm{p}$ & 1.6 & $0.25 \% \mathrm{p}$ \\
\hline \multicolumn{5}{|c|}{ (b) Estimated euro area Models } \\
\hline EA_SW03 & 6 & $-1.20 \%$ & 6 & $-0.75 \%$ \\
\hline EA_SR07 & 3 & $-0.51 \%$ & 4 & $-0.18 \%$ \\
\hline EA_QUEST3 & 2 & $-0.34 \%$ & 4 & $-0.42 \%$ \\
\hline EAES_RA09 & 1 & $-0.14 \%$ & 4 & $-0.49 \%$ \\
\hline EA_CKL09 & 1 & $-0.37 \%$ & 4 & $-0.29 \%$ \\
\hline EA_GE10 & 5 & $-0.66 \%$ & 5 & $-0.29 \%$ \\
\hline EA_GNSS10 & 3 & $-0.19 \%$ & 4 & $-0.26 \%$ \\
\hline EA_QR14 & 2 & $-0.30 \%$ & 4 & $-0.66 \%$ \\
\hline Model averages & 2.9 & $-0.46 \%$ & 4.4 & $-0.42 \%$ \\
\hline Standard deviations & 1.8 & $0.34 \% \mathrm{p}$ & 0.7 & $0.20 \% \mathrm{p}$ \\
\hline
\end{tabular}

Note: Timing refers to the quarter after the shock, when the trough or the deepest point in the response of the respective variable is reached.

as habit formation in consumption and investment adjustment cost, coupled with the presence of collateral constraints gives rise to a large initial impact of the monetary policy shock on output.

\section{A few summary statistics}

Table 7 provides some summary statistics. In the U.S. models, the trough of output following a contractionary policy shock is reached within one to six quarters, and on average in the third 
quarter. The average magnitude of the drop in output is $0.48 \%$ with a standard deviation of $0.28 \%$. Interestingly, the timing of the trough and the magnitude of the output drop in the euro area models is very similar. In the euro area, output also reaches the trough within 1-6 quarters, on average in the third quarter. The average output decline at the trough corresponds to $-0.46 \%$. Thus, it is very close to its U.S. counterpart, albeit the standard deviation of $0.34 \%$ is a bit larger.

As for inflation, the U.S. models imply that the deepest point in the inflation response occurs within 3-9 quarters, on average, in the fifth quarter. In the euro area models, the span of this range is more narrow at 4 to 6 quarters, with an average of 4.4 quarters. The average decline in inflation at the trough corresponds to $-0.30 \%$ for the U.S. models and $-0.42 \%$ for the euro area models. The respective standard deviations are very similar: $0.25 \%$ p for the U.S. and $0.20 \%$ p for the euro area.

Thus, the above comparison exercise serves to show that model averages of the predicted impact of identified policy shocks on output and inflation, are very similar for the U.S. and the euro area, in terms of timing and magnitude of the resulting contraction.

\section{Comparing implications of new macro-financial models}

\subsection{Key characteristics: Investment finance, housing finance, banking capital}

The global financial crisis has drawn attention to the need for improving the characterization of the financial sector in macroeconomic models used for business cycle and policy analysis. Many new contributions have included financial market imperfections in New Keynesian DSGE models, in particular in three areas: the financing of new investment in firms' capital for production purposes, the financing of housing investment, and the role of banks and bank capital in financial intermediation. These financial frictions help explain how the consequences of economic shocks for macroeconomic aggregates can be amplified via the financial sector, and how financial sector stress and financial crises can spill over into the real economy.

\section{Corporate investment financing and the financial accelerator.}

Fortunately, research on integrating financial frictions in macroeconomic models for policy analysis do not need to start from scratch. A prominent starting point is the so-called financial accelerator model of Bernanke et al. (1999) (BGG99). Here, the accelerator term refers to the amplification of economic fluctuations via the financial sector. Long before the global financial crisis, they already provided a tractable approach for including information asymmetries, which are central to the relationship between borrowers and lenders, in dynamic New-Keynesian models.

Lending institutions and financial contracts aim to reduce the costs of collecting information and to mitigate principal-agent problems in credit markets. By contrast, economic shocks may increase the cost of extending credit and reduce the efficiency of matching borrowers and lenders. Hence, the credit market imperfections may amplify the effects of shocks from the financial sector as well as other sectors of the economy. BGG99 focus on the financing of investment in firms' 
capital for production purposes. Their model includes risk-averse households, risk-neutral entrepreneurs and retailers. Entrepreneurs use capital and labor to produce wholesale goods. These are sold to the retailers. The retail market is characterized by monopolistic competition and price rigidities. Entrepreneurs borrow funds from households via a financial intermediary. These funds serve to pay for part of the new capital, which becomes productive in the next period. The agency problem arises because the return to capital is subject to idiosyncratic risk and can only be observed by the financial intermediary after paying some auditing cost. As a result, the entrepreneurs' net worth becomes a key factor determining their borrowing costs. Entrepreneurs with high net worth need less external funding for a given capital investment and pay lower premia. To the extent that net worth rises and falls with the business cycle, the premium to be paid for external borrowing varies counter-cyclically. Thus, it increases fluctuations in borrowing, investment, spending and production.

A version of the BGG99 model is included in MMB. The implementation differs somewhat from the handbook article because it omits entrepreneurial consumption. Its short-hand reference in MMB is NK_BGG99. The model archive also contains several more recent contributions of empirically estimated models that extend the financial accelerator mechanism of BGG99. For example, Christensen and Dib (2008) (US_CD08) extend the dynamic New Keynesian model of Ireland (2003) (see US_IR04) with a financial accelerator and estimate the model on U.S. data. In their model, debt contracts are written in nominal terms in contrast to BGG99. De Graeve (2008) (US_DG08) includes the financial accelerator in the medium-scale New-Keynesian model of Smets and Wouters (2007) (US_SW07) and estimates the extended model with Bayesian methods using U.S. data on the same non-financial macroeconomic time series as Smets and Wouters (2007). In addition, he documents a reasonably close match between the model-implied external finance premium and lower-grade corporate bond spreads. Similarly, Christiano et al. (2014) (US_CMR14) incorporate financial frictions à la BGG99 into the version of the model by Christiano et al. (2005) (US_ACEL). Unlike De Graeve (2008), they also employ financial data including the credit spread in the estimation. Furthermore, they allow the volatility of idiosyncratic productivity to vary over time. Table $\mathbf{8}$ summarizes the key features of the financial accelerator models relative to the comparison benchmark, US_SW07.

\section{Housing finance.}

Real estate booms and busts have played a central role in triggering the global financial crisis. These include not only the subprime mortgage boom and bust in the United States but also the credit-driven housing booms in a number of European countries such as Spain and Ireland. Thus, models with a more detailed housing sector that recognize the relevant financing constraints are of great interest to policy makers. 


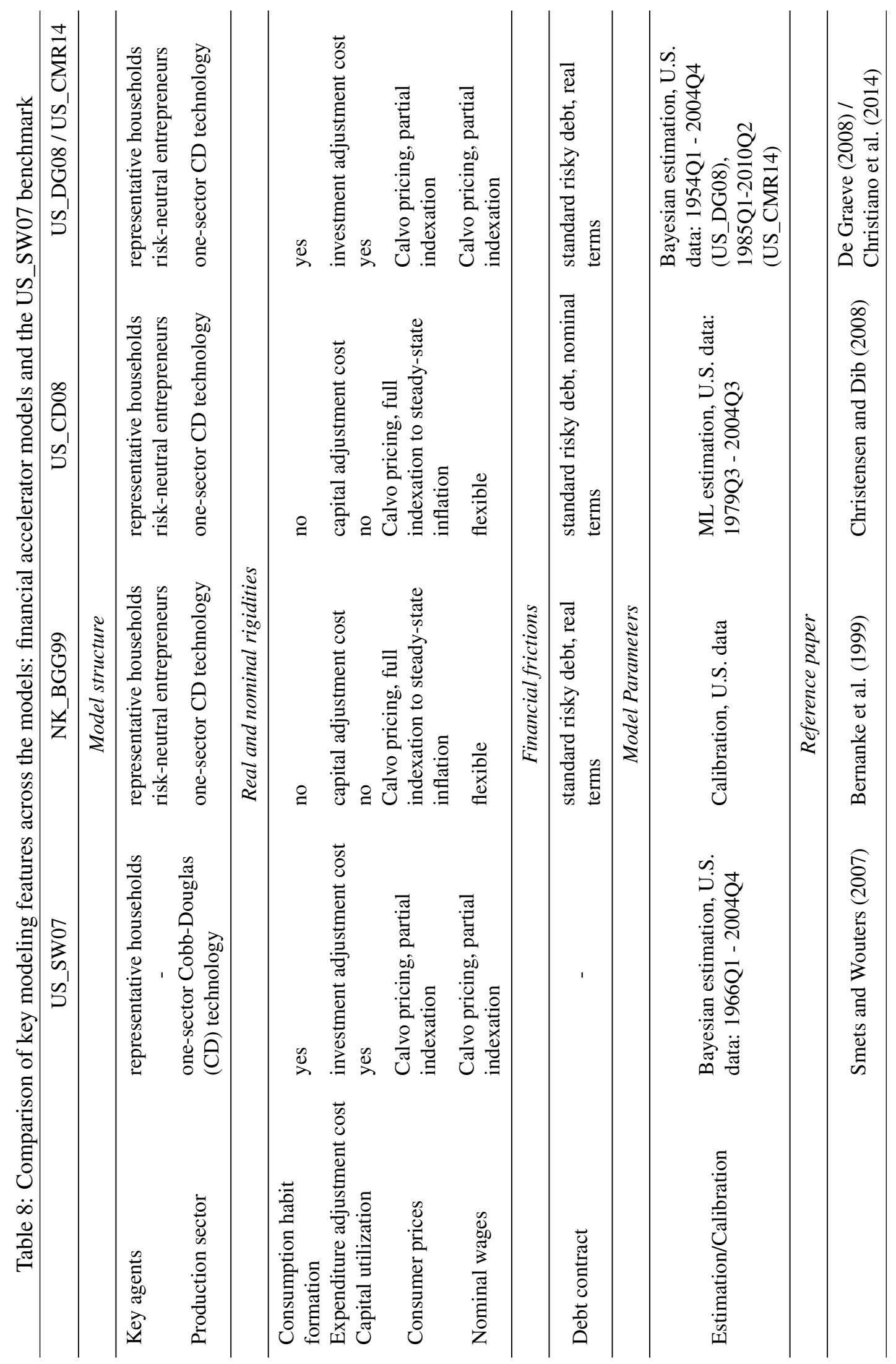


The underlying rationale of housing finance is the limited enforceability of debt contracts, as borrowers may choose to default. To overcome this limited commitment problem, lenders require collateral, typically housing and land, and provide funds only below the value of the collateral. Thus, the borrowing capacity, and hence the size of the loan is tied to the housing value. A starting point for modeling borrowing and lending under such a collateral constraint in macroeconomic models is to introduce an incentive for economic agents to act as lenders or borrowers. Technically, it is assumed that the agents differ in their discount factors: some are more patient than others. In equilibrium, the more patient ones become savers while the impatient ones become borrowers.

The collateral constraint has the following consequences: suppose an aggregate shock shifts housing demand upwards such that house prices increase. As a result, borrowing capacity expands. On this basis, the impatient agents increase expenditure on non-housing and housing goods, which in turn puts additional upward pressure on house prices. Thus, the effect of the initial shock is amplified over time due to the presence of the collateral constraint.

Kiyotaki and Moore (1997) develop a simple dynamic model with patient (and unproductive) entrepreneurs and impatient (and productive) entrepreneurs to show that the collateral channel can generate large and persistent business cycles. Iacoviello (2005) then incorporated such collateral constraints together with nominal debt in a dynamic New-Keynesian model. In his model, impatient households and entrepreneurs face collateral constraints, when borrowing funds from patient households. The both households obtain utility from housing services, while entrepreneurs use housing for the production of non-housing (consumption) goods ${ }^{12}$ The model is estimated with U.S. data and referred to as US_IAC05 in the MMB model archive.

MMB includes two other U.S. models with housing finance. The model of Iacoviello and Neri (2010) (US_IN10) features a two-sector production structure with housing and non-housing goods and imposes a collateral constraint only on impatient households. They consider various real and nominal rigidities similar to medium-scale New-Keynesian models such as Christiano et al. (2005) and Smets and Wouters (2007). The US_IN10 model is estimated on U.S. macroeconomic and housing data. The model by Kannan et al.(2012) (NK_KRS12) is a simplified version of Iacoviello and Neri (2010). Key elements of the model are the presence of financial intermediaries and the determination of the spread between the lending rate and the deposit rate. The functional form for the determination of the spread is assumed rather than derived from a micro-founded optimization problem. Financial intermediaries take deposits from patient households and lend to impatient households charging a spread that varies inversely with the net worth of borrowers. Namely, the financial accelerator mechanism operates in housing finance. Table 9 provides further information concerning key features of the three models with housing finance.

\footnotetext{
${ }^{12}$ Aggregate housing supply is assumed to be fixed.
} 


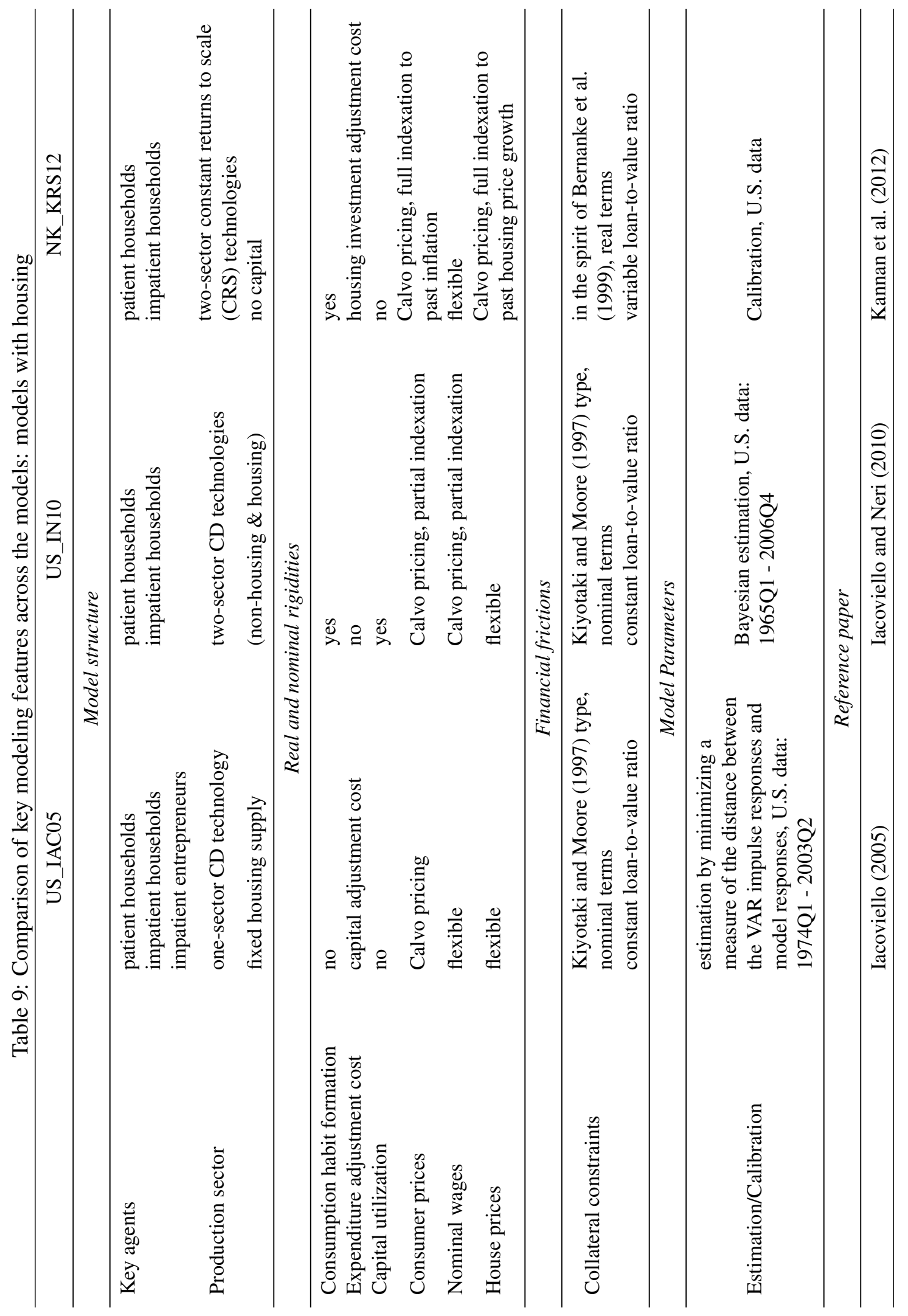




\section{Financial intermediation and bank capital.}

Banks' illiquidity, insolvency as well as counter-party risks played a prominent role during the global financial crisis, impairing credit supply by banks and thereby deepening the negative impact from excessive leverage of borrowers on the real economy. In contrast with financial accelerator and housing sector models, which focus on frictions stemming from the demand side of financial intermediation, banking sector models deal with frictions on the supply side. In these models, the balance sheet and decision processes of banks are treated explicitly. Thus, shocks originating in the banking sector can have significant spillover effects on the macroeconomy and standard, non-financial shocks can operate via new transmission channels, when macro-financial linkages are taken into account. In what follows, we focus on three quantitative monetary DSGE models in which banking capital plays a key role.

In the model of Gertler and Karadi (2011) (NK_GK11), banks obtain short-term funds from households and lend them to non-financial firms by purchasing the firms' long-term securities. There is no financial friction between banks and non-financial firms. Instead, the possibility that the banker can divert part of the bank' assets creates a moral hazard problem between the bank and households. In order to induce households to provide funds, the bank has to satisfy an incentive constraint: the pecuniary benefit from diverting funds must be at least as small as the gain from staying in business. This condition serves as an endogenous constraint on the bank's leverage. Such financial intermediaries are imbedded into an otherwise standard medium-scale New Keynesian model such as Christiano et al. (2005).

Meh and Moran (2010) (NK_MM10) use the double moral hazard framework of Holmstrom and Tirole (1997) and introduce banking decisions via an optimal financial contract. The first moral hazard problem is between a representative household and a representative bank. As the bank's monitoring technology is not directly observed by the investor, the latter requires the bank to participate in the project with its own net worth to mitigate this information asymmetry. Therefore, the ability of the bank to attract loanable funds depends on its capital position. The second moral hazard problem is between the bank and the entrepreneur, because entrepreneurial effort is private information. The bank requires entrepreneurs to participate financially, i.e. "to put some skin in the game". The double moral hazard problem is then incorporated within a standard New Keynesian framework.

In Gerali et al. (2010) (EA_GNSS10), banks channel funds from patient households to entrepreneurs and impatient households. Meanwhile, the bank faces a leverage constraint as a form of paying a pecuniary cost whenever its net worth to asset ratio moves away from an exogenously given target. The bank's optimal decision implies that credit supply depends positively on the bank net worth. In addition, banks have monopolistic power to set deposit and loan rates. These rates exhibit stickiness due to adjustment costs. The banking sector is included in a model with collateral constraint à la Iacoviello (2005). While the preceding two models are calibrated, the EA_GNSS10 model is estimated on the euro area macroeconomic data. Table 10 summarizes the key features of the models with bank capital. 


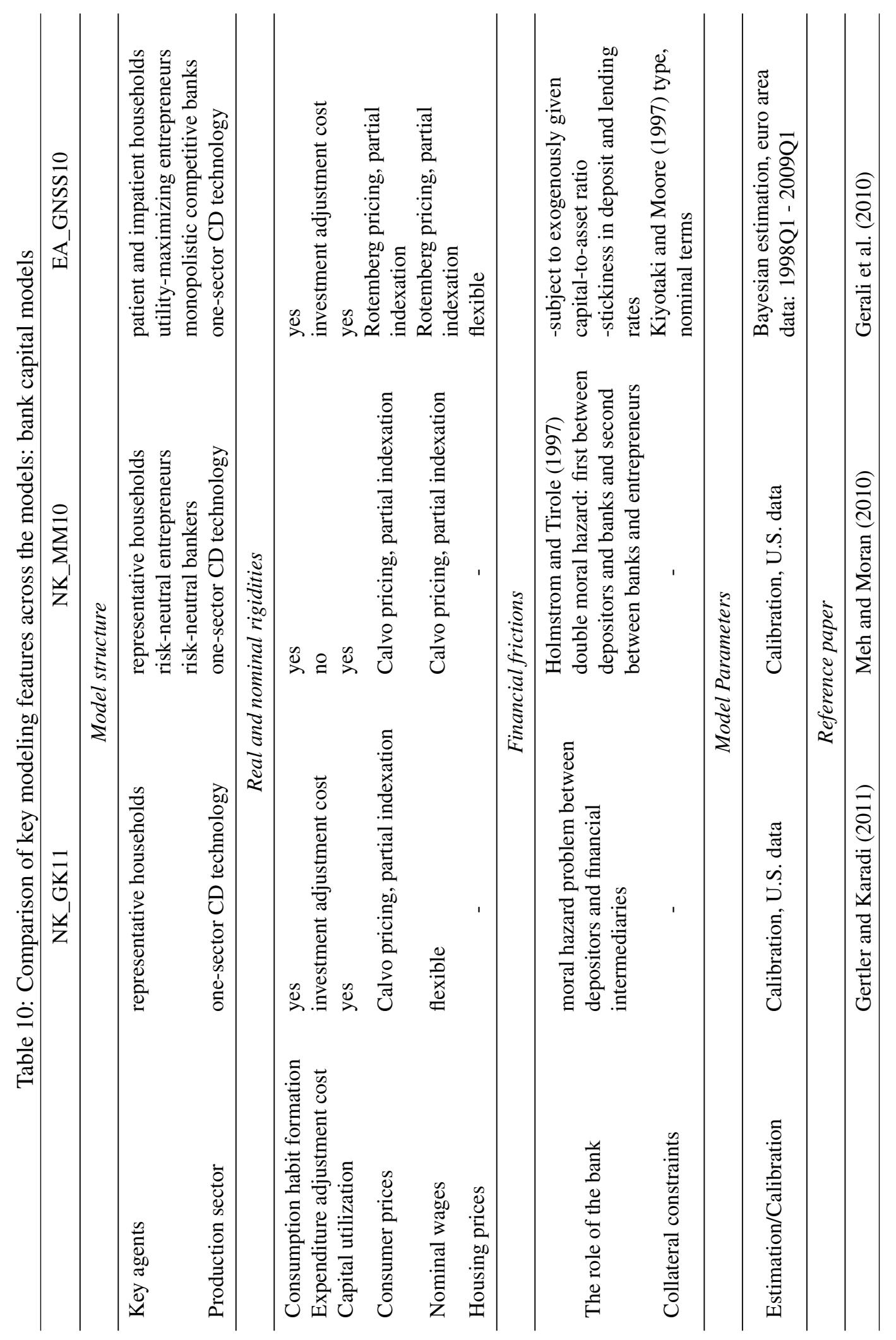


Exploring how the financial sector propagates and amplifies disturbances.

In the following, we use MMB to explore and compare the dynamics of the above-mentioned macro-finance models. For models with financial accelerator on corporate investment and models with housing finance, we compare impulse response functions to a monetary policy shock, a general technology shock and shocks that are more akin to aggregate demand shocks. Here, we extend the model comparison approach outlined in Section 3 by utilizing some economic shocks as common shocks in the models considered. The Smets and Wouters (2007) model (US_SW07) serves as a benchmark for comparison. Furthermore, we use the monetary policy rule estimated by Smets and Wouters (2007) as the common policy rule for all models. In this manner, we can isolate differences due to structural assumptions of each model from differences due to different assumptions on monetary policy. The SW rule is given in equation 110 . For models with a role of bank capital, we simulate the original model to investigate the effects of an unexpected reduction in bank capital.

\subsection{Propagation mechanisms: Investment financing and the financial accelerator}

Striking differences in amplification of the effect of monetary policy.

To begin, we compare the transmission of the monetary policy shock in the four models with financial accelerator effects due to information asymmetries in the financing of corporate investment, (NK_BGG99, US_CD08, US_DG08 and US_CMR14), relative to the benchmark (US_SW07). Figure 12 displays the effects of an unanticipated increase in the nominal interest rate of one percentage point for the commonly defined macroeconomic aggregates. In all four models, the nominal interest rate increases while output and inflation decline. The standard channel of monetary transmission is reflected in higher real interest rates that lead households to reduce consumption today and firms to refrain from investment.

The financial accelerator mechanism is at work in all four models that contain financial frictions. As can be seen from Figure $\mathbf{1 3}$ firms' net worth falls due to a reduction in the price and return of capital $\left[13\right.$ Borrowing needs and leverag $e^{14}$ of entrepreneurs increase, and the external finance premium (EFP) rises, depressing investment. The US_CD08 model, where the financial contract is in nominal terms, also exhibits a debt-deflation mechanism.

Yet, the magnitude, timing and dynamic pattern of responses differ substantially across models. It is particularly striking that the smaller New Keynesian models NK_BGG99 and US_CD08 display much stronger responses of output and inflation and a much smaller response of the nominal interest rate than the medium-size DSGE models US_SW07, US_DG08 and US_CMR14. This diversity of responses to a monetary policy shock stands in contrast to the findings of Taylor and Wieland (2012). The estimated medium-size DSGE models with financial accelerator US_DG08 and US_CMR14 still remain close to the other medium-size models, although the response of

\footnotetext{
${ }^{13}$ Note that the financial variables have not been redefined as common variables. Thus, the differences can only be interpreted qualitatively. Yet, the impact on GDP is directly comparable.

${ }^{14}$ Leverage is defined as ratio of the value of capital $Q_{t} K_{t}$ to the entrepreneur net worth.
} 
Figure 12: Impulse Responses to a Contractionary Monetary Policy Shock UnDER SW RULE: MACRO VARIABLES
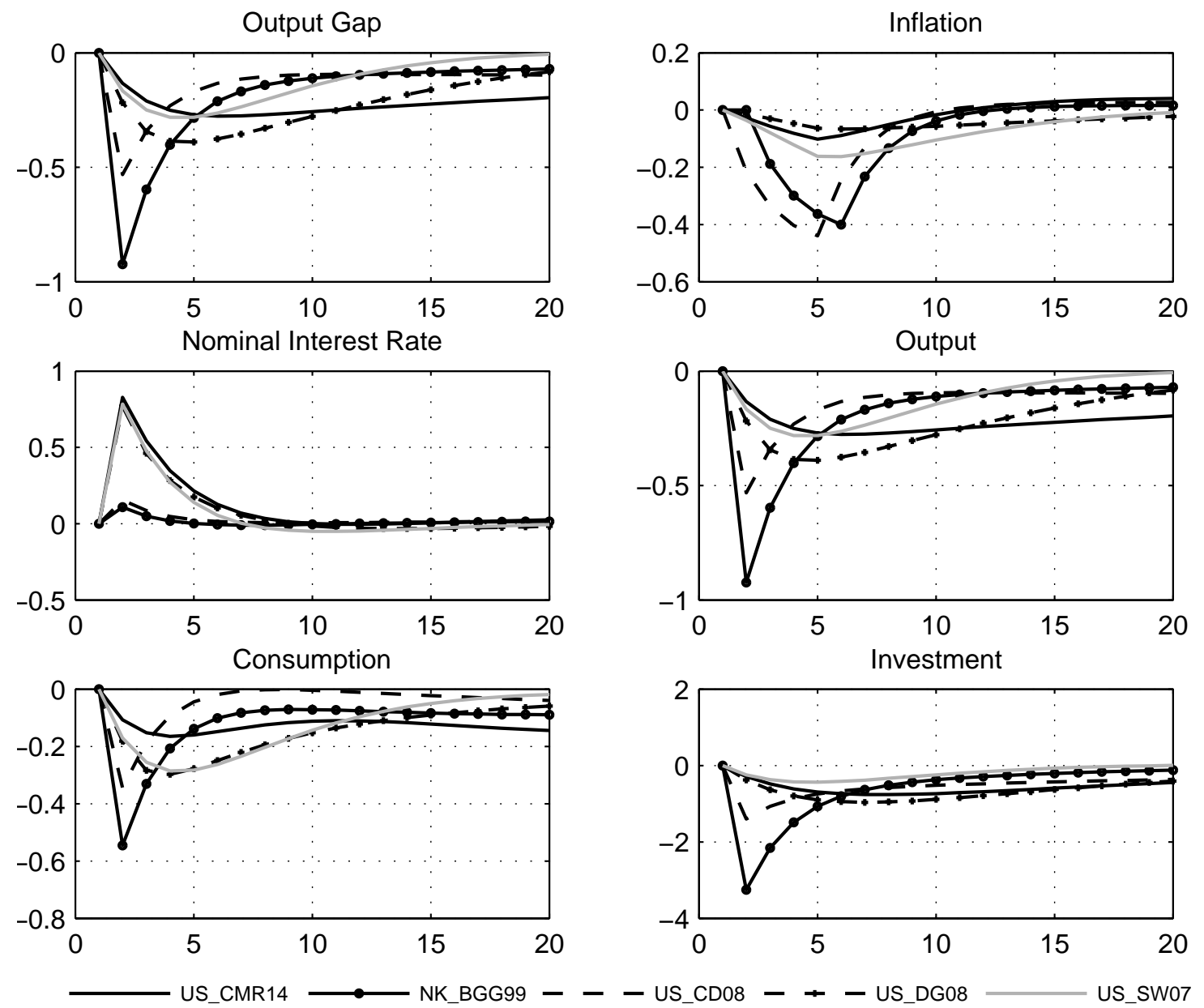

Notes: Horizontal axis represents quarters after the shock. Units of the vertical axis are percentage deviations from steady-state values. Inflation is the rate of inflation over the previous four quarters. Nominal interest rate is annualized. Other variables are expressed in quarterly terms.

output in US_CMR14 is substantially more persistent as discussed in Section $\mathbf{5 . 2}$.

In US_DG08, investment responds more strongly to the unexpected policy tightening than in US_SW07 due to the financial accelerator effect 15 The effect on consumption remains very similar. In sum, the impact on GDP is magnified a bit. GDP declines by about 40 basis points relative to 30 basis points in US_SW07. There is no similar magnification effect, when output responses in US_SW07 and US_CMR14 are compared. The reasons are a somewhat smaller consumption response and a weaker transmission of the financial accelerator channel to investment in US_CMR14. In particular, the response of investment in US_CMR14 is less pronounced than in US_DG08 due to higher curvature of the investment adjustment cost function.

\footnotetext{
${ }^{15}$ Noteworthy, estimates of the curvature of investment adjustment costs function are almost identical in US_SW07 and US_DG08.
} 


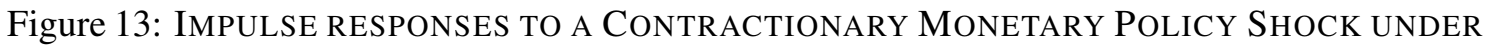
SW Rule: FinANCIAL VARiables
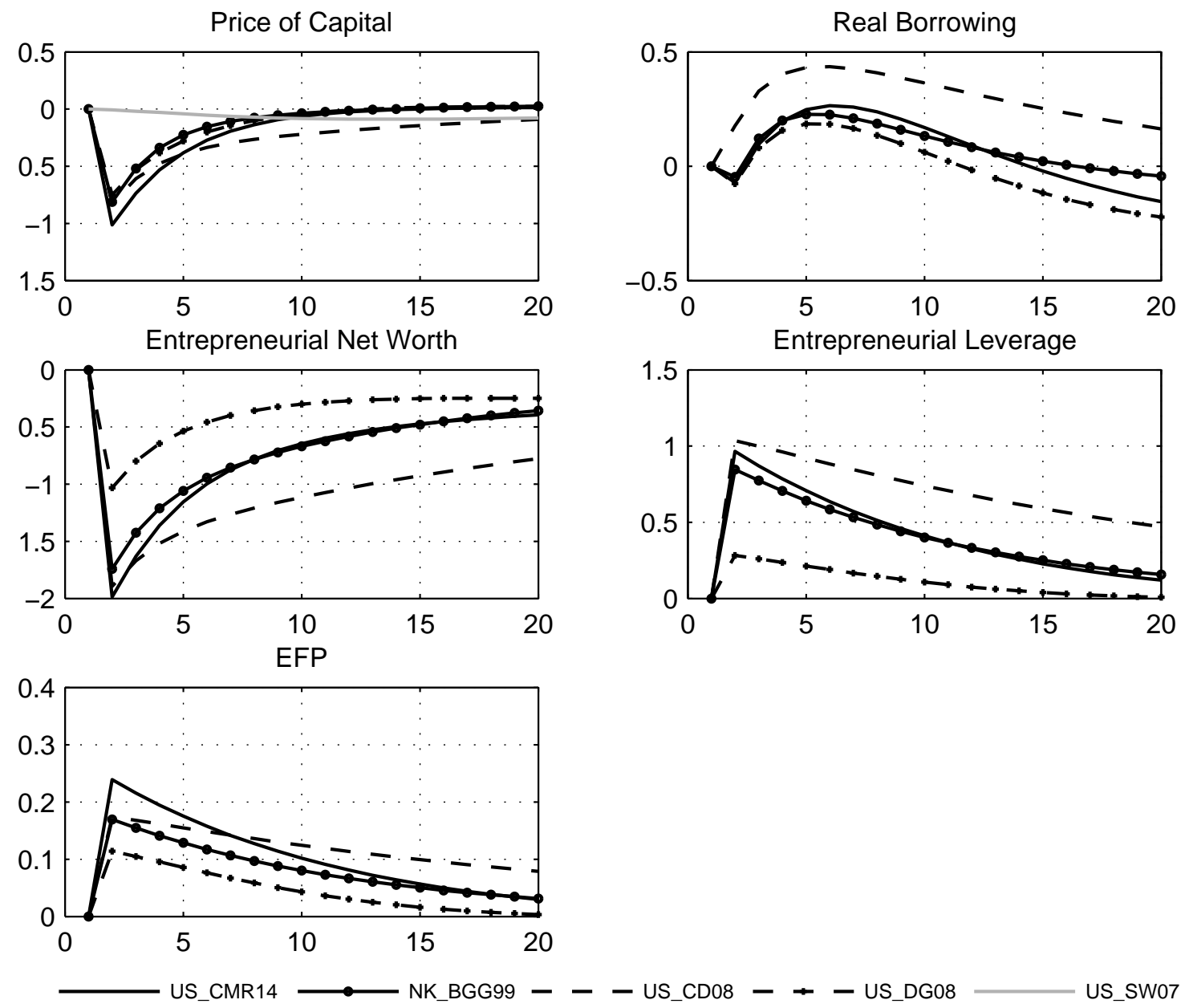

Notes: Horizontal axis represents quarters after the shock. Units of the vertical axis are percentage deviations from steady-state values. EFP(External Finance Premium) is annualized. Other variables are expressed in quarterly terms.

Investment adjustment costs attenuate sharp responses the the external finance premium

Where does the big difference in GDP effects between medium-size DSGE models and smaller models with financial accelerator come from? The reason is the different working of the financial accelerator effect on investment in the two smaller models. The sharp increase in the external finance premium translates directly into a sharp reduction in investment in all financial accelerator models. In US_DG08 and US_CMR14 the response of investment is hump-shaped and persistent, reaching a substantially lower peak effect than in NK_BGG99 and US_CD08. This is due to different specifications of adjustment costs across models: US_DG08 and US_CMR14 assume investment adjustment costs (as in Christiano et al.(2005)), whereas NK_BGG99 and US_CD08 assume capital adjustment costs. As in US_DG08 and US_CMR14 it is costly to adjust the flow of 
investment, forward looking agents adjust investment already today in expectation of an increase in the external finance premium. Accordingly, fluctuations in the premium have a smaller effect on the economy under investment adjustment costs than under capital adjustment costs ceteris paribus (see De Graeve (2008)). One might also ask why the largest impact on GDP occurs in NK_BGG99, rather than in US_CD08, where the financial accelerator is reinforced by a debtdeflation mechanism. This has to do with the calibration of capital adjustment costs. It is less costly to adjust capital in NK_BGG99 than in US_CD08.

Given the importance of the capital versus investment adjustment cost assumption and the striking differences it implies for output responses, one might ask which of the assumptions would is supported by the data. We compare impulse responses of output from the models with the empirical impulse responses stemming from a vector autoregression (VAR). To this end, we estimate a VAR, using the same observables and recursive identification as in Christiano et al. (2005) on the sample 1965Q3 - 2007Q3 ${ }^{16}$ Figure 14 presents impulse responses of the Federal Funds Rate and real GDP to a one percentage point increase in the monetary policy rate. The Federal Funds Rate increases on impact by 1 percentage point and then gradually declines. Real GDP exhibits a hump-shaped response, reaching a trough 6 quarters after the shock. This dynamic pattern of GDP response is consistent with investment adjustment costs assumption. Noteworthy, the VAR-based median response of output in the trough period is quantitatively very close to the model-average of the trough output effect in the U.S. estimated models reported in Table 7

\section{Sharp GDP responses trigger strong contemperaneous policy feedback.}

Another difference between the medium-size models and the smaller models concerns the behavior of the nominal interest rate (see Figure 12. In US_DG08, US_CMR14 and US_SW07 the nominal interest rate increases by about 1 percentage point in response to the policy shock as one might have expected. By contrast, the interest rate rises by less than 20 basis points in NK_BGG99 and US_CD08. In these two models monetary policy has a strong contemporaneous effect on GDP growth that feeds back to the interest rate via the contemporaneous response to GDP growth in the SW rule. At first sight, this finding appears odd, particularly in light of the simulation of monetary policy shocks reported in Bernanke et al. (1999) which indicates a much stronger within-quarter effect of the policy shock on the interest rate. However, it turns out that the model dynamics are quite different under the original monetary policy rule. To illustrate this effect, we simulate all the other models under the original policy rule from Bernanke et al. (1999) (BGG99 rule) ${ }^{17}$ The rule is given by:

$$
i_{t}^{z}=0.9 i_{t-1}^{z}+0.11 p_{t-1}^{z}+\eta_{t}^{i}
$$

\footnotetext{
${ }^{16}$ The order of variables in the vector of observables is as follows: real GDP, real consumption, the GDP deflator, real investment, real wage, labor productivity, Federal Funds Rate, the change in M2 real money stock and real profits. The lag length is set to 2 quarters based on the Akaike Information Criterion. The VAR model also includes an intercept and a linear trend. The confidence bands are obtained by bootstrapping with 500 draws.

${ }^{17}$ Simulations are carried out in the MMB menu (One policy rule, many models) by assigning the policy rule coefficients under the tab "User-specified rule".
} 
Figure 14: IMPULSE RESPONSES TO A ONE PERCENTAGE POINT INCREASE IN THE FEDERAL FUNDS RATE IN A STRUCTURAL VAR
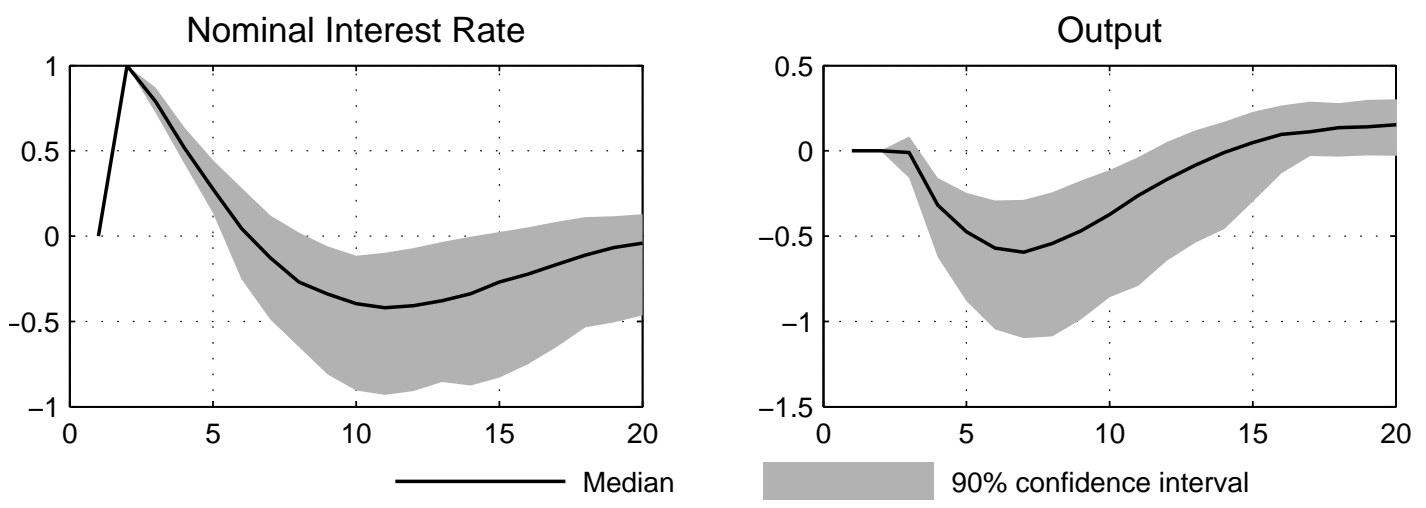

Notes: The variables and recursive identification are consistent with Christiano et al. (2005) on the sample of 1965Q32007Q3. The horizontal axis represents quarters after the shock. The solid lines refer to the median impulse responses. Shaded areas represent the $90 \%$ confidence intervals obtained by bootstrapping.

where $i_{t}^{z}$ refers to the annualized short-term interest rate; $p_{t}^{z}$ is the annualized quarter-to-quarter rate of inflation and $\eta_{t}^{i}$ refers to the common monetary policy shock. As shown in Figure 15 the strong contemporaneous feedback to the nominal interest rate disappears when simulating this rule with lagged inflation. Since this rule implies no reaction to the current state of the economy, the resulting impact of the policy shock on output and inflation is much greater.

Figure 15: IMPUlSE RESPONSES TO A CONTRACTIONARY MONETARY POLICY SHOCK UNDER BGG99 RULE: NOMINAL INTEREST RATE AND OUTPUT
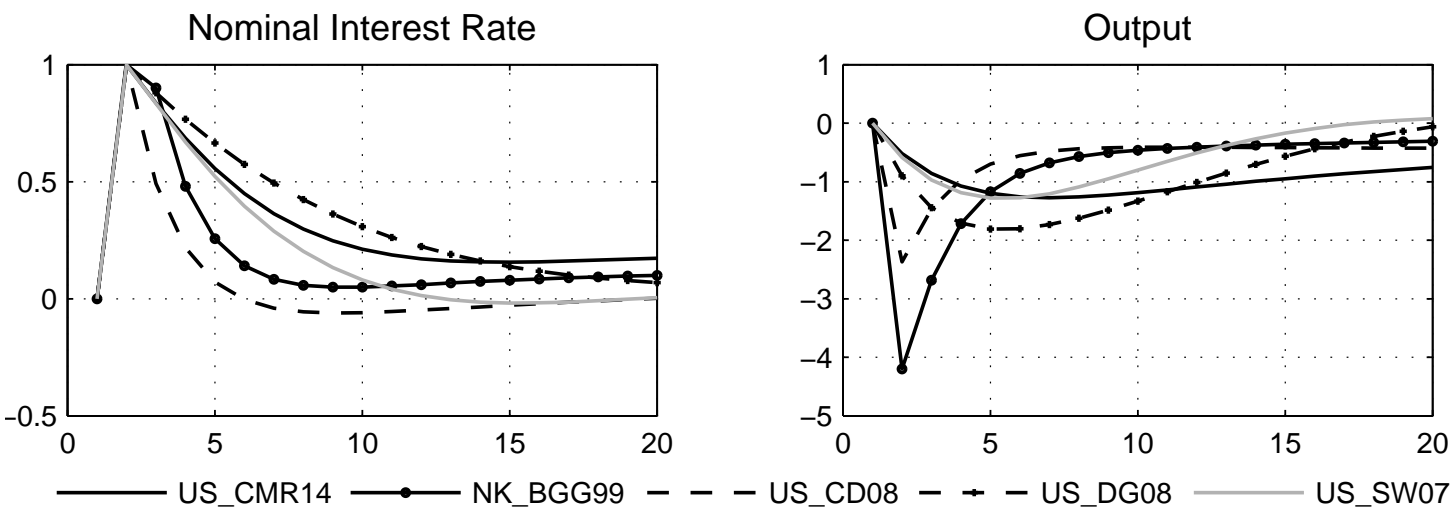

Notes: Horizontal axis represents quarters after the shock. Units of the vertical axis are percentage deviations from steady-state values. Nominal interest rate is annualized. Output is expressed in quarterly terms.

The sensitivity of interest rate dynamics to the timing assumption of the policy rule in the two smaller models suggests that the specification of dynamics in these models is not rich enough to 
be used to assess the transmission of monetary policy in a quantitative manner for policy purposes. It indicates the usefulness of building and estimating medium-size DSGE models for this purpose. Interestingly, the five medium-size models considered here continue to indicate fairly similar GDP impact of policy shocks under the rule from NK_BGG99 (US_SW07, US_DG08 and US_CMR14 are shown in Figure 15, but not G7_TAY93 and US_ACEL).

\section{Financial accelerator or decelerator of productivity disturbances?}

Figures 16 and 17 report on the impact of a positive one-percent technology shock 18 The degree of exogenous persistence of this shock is assumed to be identical in the models considered. In particular, we set the common persistence parameter of the AR(1)-technology process to 0.9. Again, the common monetary policy rule corresponds to the estimated interest rate rule in US_SW07.

In all four models output increases in response to such technological progress. This increase is also visible in investment and consumption. Due to the rigidity of price adjustment, and in the case of the US_SW07 and US_DG08 models also nominal wage adjustment, actual output increases less than the level output that would be realized under flexible prices. For some time, a gap opens up between actual output and this measure of potential output ${ }^{19}$ The negative output gap leads to a decline in inflation. The SW rule then calls for monetary easing and the nominal interest rate declines.

With regard to the financial accelerator effect, the price of capital, firms' net worth and real borrowing increase in response to the technology shock. As leverage first declines and then rises, so does the external finance premium. Magnitudes and dynamic patterns differ. Again, the NK_BGG99 and US_CD08 indicate a sharp positive impact of the change in financial variables on firms' investment. Investment and output dynamics in US_SW07, US_DG08 and US_CMR14 follow a hump-shaped pattern departing from and returning to steady state more slowly than in the other two models. The presence of investment adjustment costs in the medium-size models explains the more sluggish responses than in the NK_BGG99 and US_CD08 models that assume capital adjustment costs. Bernanke et al. (1999) showed that the financial accelerator amplified the effect of technology shocks on investment and GDP relative to the benchmark without the financial friction. The model of De Graeve (2008) delivers the opposite result. Relative to the model without the financial friction, the financial accelerator mechanism added by De Graeve (2008) actually dampens the investment and GDP response to a technology shock. As the demand for and price of capital increase, investment stays high for some time. The value of the capital stock then outgrows net worth and increases borrowing needs for quite some time. Accordingly, the external

\footnotetext{
${ }^{18}$ For comparison, the size of the shock in each model is scaled such that it would increase output on impact by one percent in the absence of endogenous responses of other variables.

${ }_{19}$ De Graeve (2008) defines potential output as the level of output under flexible prices and in absence of financial frictions. For direct comparability with the other financial accelerator models US_CD08 and NK_BGG99, we employ a common definition of potential output - under flexible prices and in the presence of financial frictions - also for US_DG08. The results are, however, not sensitive to the definition of potential output in this case.
} 
Figure 16: Impulse ReSPonses to A Positive TeChNOlogy SHock UNDER SW RULE: MACRO VARIABLES
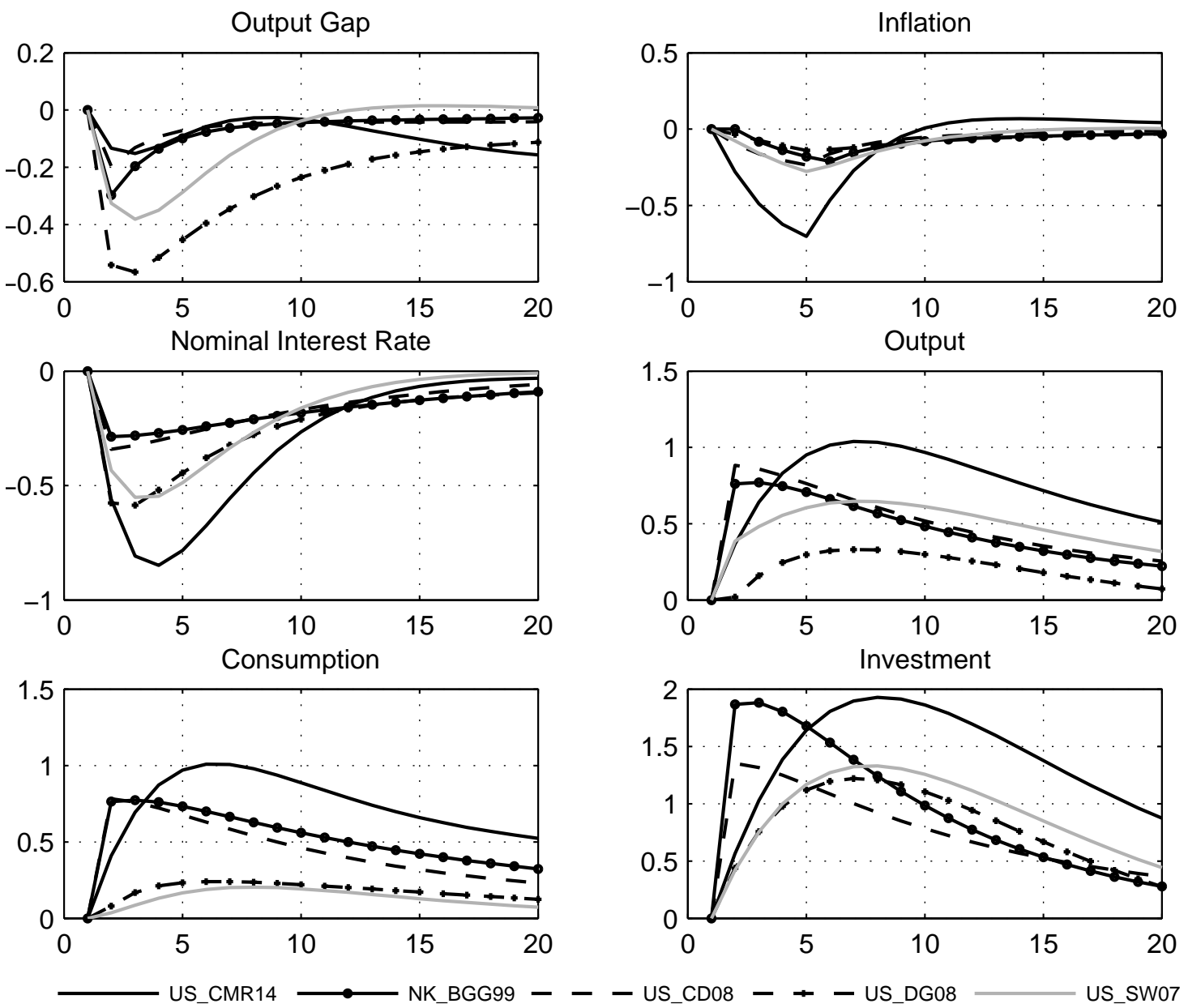

Notes: Horizontal axis represents quarters after the shock. Units of the vertical axis are percentage deviations from steady-state values. Inflation is the rate of inflation over the previous four quarters. Nominal interest rate is annualized. Other variables are expressed in quarterly terms.

finance premium rises. As De Graeve (2008) notes, because long-lasting positive investment will be costly due to a high future premium for external finance, investment will be lower in all periods than otherwise. Indeed, the investment response in US_DG08 is smaller relative to US_SW07, which also features investment adjustment costs but no financial friction. However, this is not the case for US_CMR14, that is structurally very close to US_DG08 and yet exhibits larger responses of consumption, investment and hence output relative to US_SW07.

\section{Estimated parameters of price stickiness make a difference for the accelerator effect}

A key parameter for investment dynamics is the curvature of the investment adjustment costs. In US_SW07 and US_DG08, this parameter is almost identical (5.76 and 5.77, respectively), whereas in US_CMR14 it is estimated to be substantially higher (10.78). Ceteris paribus, a higher 
Figure 17: Impulse Responses to a Positive TeChnology Shock Under SW Rule: FinANCIAL VARIABLES
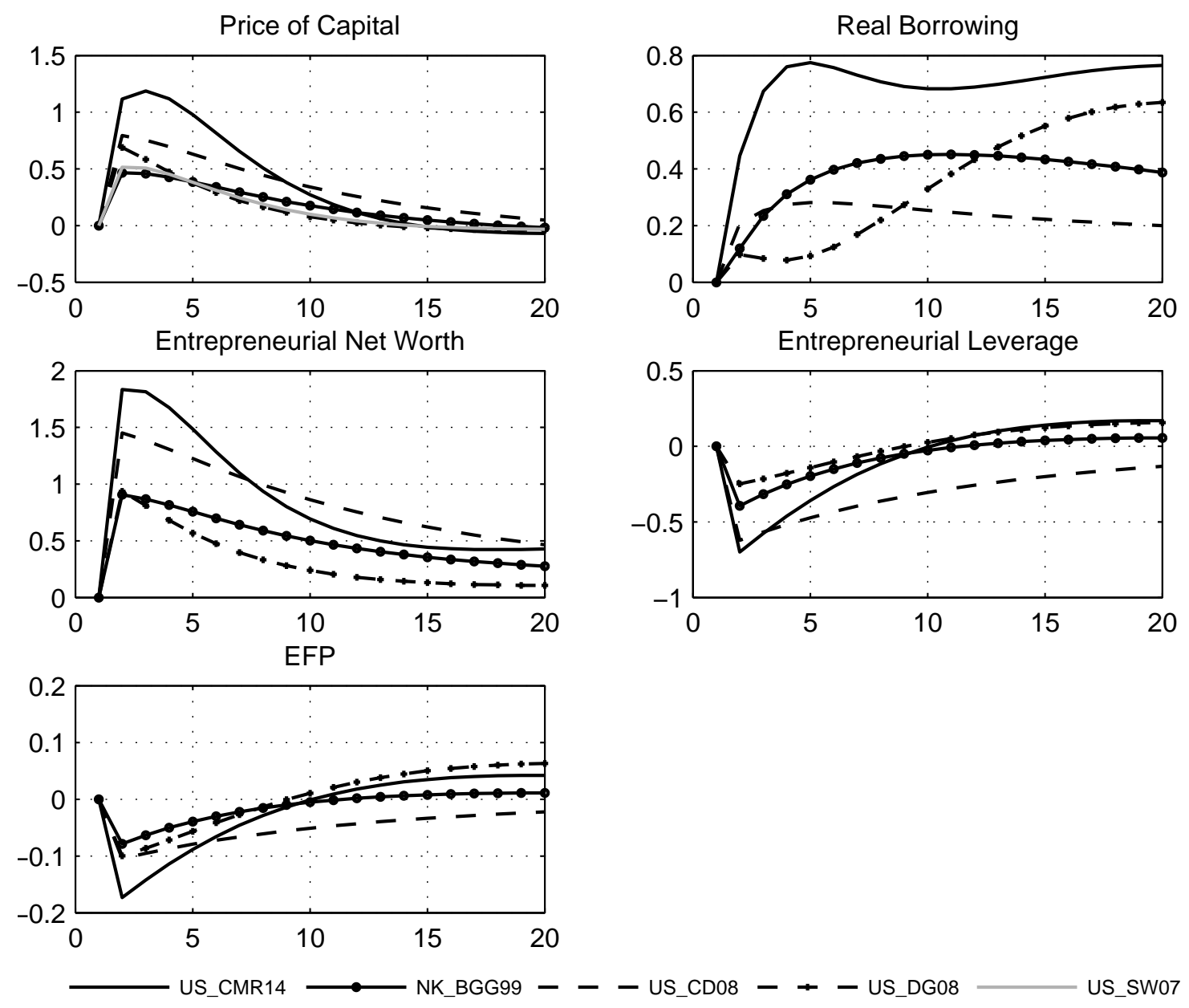

Notes: Horizontal axis represents quarters after the shock. Units of the vertical axis are percentage deviations from steady-state values. EFP(External Finance Premium) is annualized. Other variables are expressed in quarterly terms.

curvature makes the adjustment process costlier, dampening the investment response. Yet, the investment response in US_CMR14 is stronger than in the other models.

Another important parameter is the degree of price stickiness. In US_DG08, the probability that firms will not be able to reset the price, is estimated at 0.92 , whereas the corresponding estimate in US_SW07 is 0.65 and 0.74 in US_CMR14. In other words, prices are more flexible in US_SW07 and US_CMR14. The degree of price flexibility determines the strength of response of consumption and investment variables to a technology shock, because it determines the dynamics of the real interest rate. Conditional on the monetary policy rule, which is common for all models in this exercise, more flexible prices imply that inflation will ceteris paribus fall by more in response to a technology shock, causing the central bank to loosen the nominal rate by more. As a result, the real interest rate is lower for more flexible prices. Thus, consumption increases more 
for more flexible prices. Also investment rises more substantially. In a model without financial friction such as US_SW07, equilibrium requires the real rate to move together with the aggregate return on capital. Therefore, in an economy with more flexible prices, capital increases by more ceteris paribus, causing a higher investment response. With the financial accelerator, a lower real rate translates into a lower external finance premium, strengthening entrepreneurial net worth and thereby also supporting the investment boom.

To sum up, greater price stickiness dampens the responses of consumption and investment to technology shocks. This is a further reason why the responses in US_DG08 are smaller relative to the model without the financial friction - US_SW07. The other important reason is the presence of the 'decelerator' effect, as described in De Graeve (2008). The differences in price stickiness also explain why consumption and investment responses are stronger in US_CMR14 when compared to US_DG08.

With regard to the earlier findings of Bernanke et al. (1999) it is noteworthy to point out the sensitivity to the assumption for the monetary policy rule and the persistence of the technology process. They use a random walk process for technology. In this case, a shock has very large and persistent effects on output. Consequently, actual output exceeds potential output and inflation goes up.

\section{Investment-specific shocks.}

We have also simulated and compared the impact of investment-specific shocks in the US_SW07, US_DG08 and US_CD08 models. De Graeve (2008) calls this shock an investment supply shock, since it causes investment to increase and the price of capital to decrease. Smets and Wouters (2007) group it under (aggregate) demand shocks because they lead to an increase in both output and inflation. In this context, it is of interest to note that such investment-specific shocks play an important role in explaining the Great Recession following the global financial crisis when the US_SW07 model is extended to cover this period (see Wieland and Wolters (2013)). Conditional on the model parameterization, the financial friction included in the US_DG08 and US_CD08 models dampens the impact of such investment shocks on investment and GDP.

\subsection{Propagation mechanisms: Housing finance and credit booms}

Next, we compare the effects of monetary and technology shocks in the three models with housing finance, US_IAC05, US_IN10 and NK_KRS12, relative to the US_SW07 model as benchmark 20 In addition, we examine the impact of demand shocks originated from housing sector on the broad economy.

\footnotetext{
${ }^{20}$ The components of aggregate consumption and investment differ in the models with housing due to different assumptions on the production sector and housing market. In the US_IN10 and NK_KRS12 models, aggregate consumption consists of the consumption of patient and impatient households, and investment is defined as the sum of nonresidential and residential investment. Meanwhile, in the US_IAC05 model, aggregate consumption includes the consumption of entrepreneurs additionally to two types of households' consumption and investment is nonresidential investment.
} 


\section{Monetary transmission via housing finance.}

Figure 18 shows the consequences of a contractionary monetary policy shock on macro variables. Qualitatively, the three models with housing finance exhibit the same Keynesian-style features as the benchmark. Due to price rigidities, the contractionary monetary shock induces an increase in the real interest rate, output declines below its flexible price leve 21 and this gap causes lower inflation. Both, consumption and investment decrease. Quantitatively, the impact on real GDP is much sharper and more pronounced in the US_IAC05 and US_IN10 models. The NK_KRS12 model, however, is closer to the US_SW07 benchmark. The latter two models exhibit more muted and hump-shaped responses of GDP and its components, consumption and investment.

Figure 19 displays the transmission of the monetary shock via housing finance. The collateral constraints on nominal borrowing in the US_IAC05 and US_IN10 models magnify the effect of unanticipated policy tightening. As inflation falls and real house prices decrease, the debt capacity of borrowers is reduced. In the US_IAC05 model impatient households and entrepreneurs are both borrowing-constrained. Accordingly, the impatient households cut back further on consumption, while the entrepreneurs reduce non-residential investment along with consumption. Likewise, in the US_IN10 model impatient households curtail consumption by more. Moreover, residential investment declines significantly, because sticky wages in combination with flexible house prices intensify the effect of a monetary shock on output in the residential sector. Meanwhile, output shows no hump-shaped responses in these two models. The reasons is that the US_IAC05 model exhibits no habit formation in consumption and only small capital adjustment costs, while the he US_IN10 model features no capital adjustment costs.

The NK_KRS12 model exhibits a more flexible collateral constraint. This generates less amplification than the standard collateral constraints used in the other models with housing. A higher loan-to-value ratio in this model is accompanied by a rise in lending rates. By contrast, the amount of borrowing is restricted to a certain fraction of collateral in case of the standard collateral constraint. Accordingly, a fall in the collateral value leads directly to the reduction of borrowing. In the NK_KRS12 model, impatient households still take out more loans even with higher interest rate in response to a contractionary monetary shock. This dampens the responses of consumption and residential investment. Furthermore, since there is no capital in this model, aggregate demand lacks nonresidential investment which is an interest-sensitive component of GDP. Overall, the impact of the monetary shock on output is smaller in the NK_KRS12 model than in the other models.

As in the case of the NK_BGG99 and US_CD08 models in the preceding subsection, we find that due to insufficient real rigidities, the US_IAC05 and US_IN10 models exhibit a sharp contemporaneous response of output that strongly feeds back via the SW rule to the contemporaneous

\footnotetext{
${ }^{21}$ In the NK_KRS12 model, the potential output is defined as the level of output that could be realized without nominal and financial frictions.
} 
Figure 18: IMPULSE RESPONSES TO A CONTRACTIONARY MONETARY POLICY SHOCK UNDER SW RUle: Macro VARiables
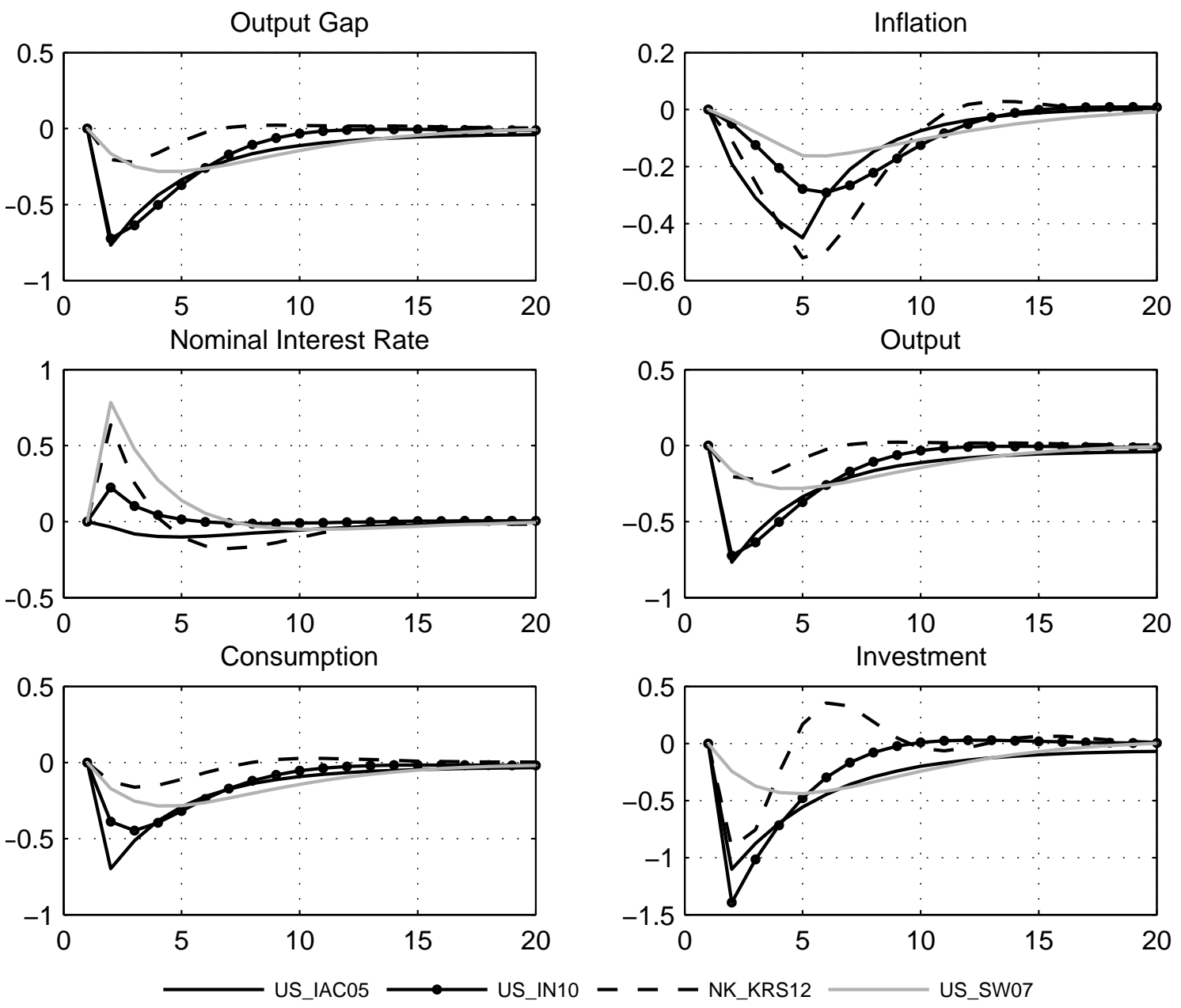

Notes: Horizontal axis represents quarters after the shock. Units of the vertical axis are percentage deviations from steady-state values. Inflation is the rate of inflation over the previous four quarters. Nominal interest rate is annualized. Other variables are expressed in quarterly terms.

nominal interest rate. For the US_IAC05 model the positive monetary policy shock implies a slight decline in the nominal interest rate. Similarly to the financial accelerator models analyzed earlier, this strong contemporaneous effect disappears when the models are simulated under the policy rule of Bernanke et al. (1999) (see Figure 20).

The sensitivity of interest rate dynamics to the timing assumption of the policy rule suggests that the dynamics in these models are not rich enough to be used to assess the transmission of monetary policy in a quantitative manner as in the case of medium-size DSGE models with more sources of endogenous persistence. Thus, the comparison of monetary transmission mechanisms in the two groups of macro-financial models supports including habit formation in consumption 
Figure 19: IMPULSE RESPONSES TO A CONTRACTIONARY MONETARY POLICY SHOCK UNDER SW RULE: INVESTMENT \& FINANCIAL VARIABLES
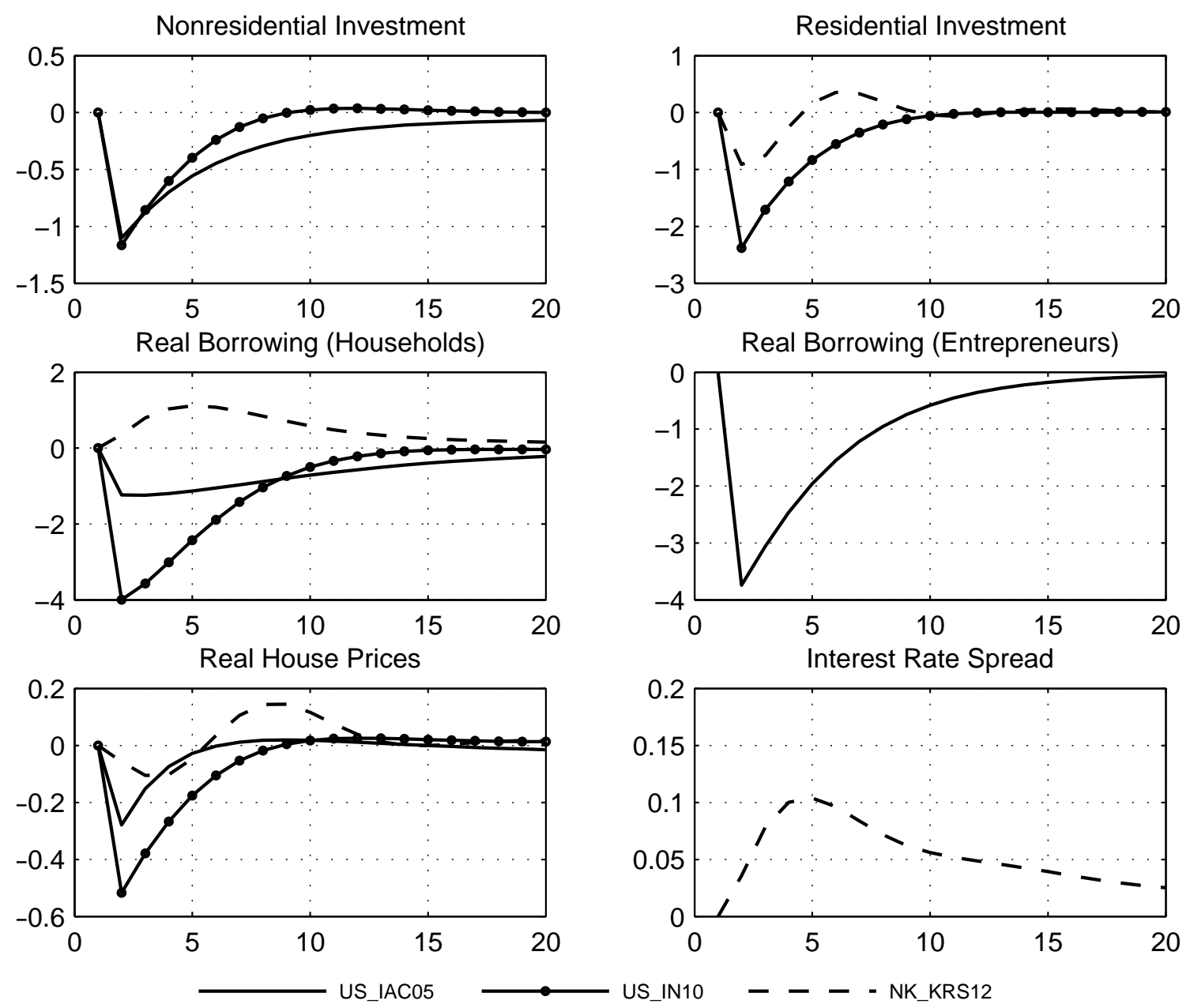

Notes: Horizontal axis represents quarters after the shock. Units of the vertical axis are percentage deviations from steady-state values. Interest rate spread is annualized. Other variables are expressed in quarterly terms.

and investment adjustment costs in models for quantitative monetary policy analysis.

General technological progress and housing finance.

We also examine effects of a common technology shock in the housing finance models. The shock has a common autocorrelation coefficient of 0.9. In the US_IN10 and NK_KRS12 models, which contain two production sectors, the shock increases the total factor productivity in the nonresidential (consumption goods) sector. Figures 21 and 22] present the impact of a one percent increase in such a shock.

As in the US_SW07 model, GDP rises and CPI inflation declines in response to a positive technology shock in the models with housing. It leads to a housing boom without inflation, which is amplified by collateral constraints. 
Figure 20: Impulse Responses to a Contractionary Monetary Policy Shock Under BGG99 RULE: NOMINAL INTEREST RATE AND GDP
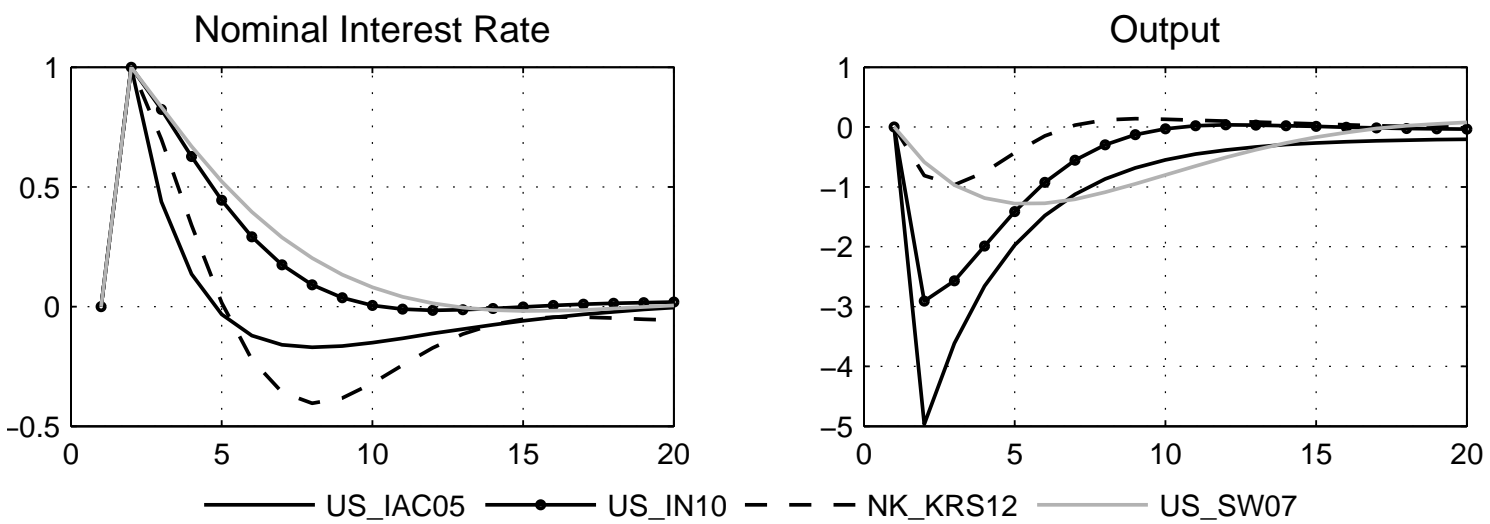

Notes: Horizontal axis represents quarters after the shock. Units of the vertical axis are percentage deviations from steady-state values. Nominal interest rate is annualized. Output is expressed in quarterly terms.

The persistent but temporary increase of productivity in the nonresidential sector is followed by a lower real interest rate so that aggregate demand is equated to the expanded aggregate supply. The reduction of the real rate causes real house prices to rise, which in turn increases the borrowing capacity of collaterally-constrained agents. This allows borrowers to obtain more funds, which are either consumed or invested. The amplifying effect of the collateral channel is most apparent in the responses of consumption. Consumption increases two or four times more in the housing finance models than in the US_SW07 model. Though the decline in inflation reduces collateral values, the collateral channel outweighs the debt deflation channel.

Surprisingly, the output gap in the US_IN10 model increase, whereas CPI inflation declines. When house prices rise, the combination of flexible house prices and sticky wages in the residential sector increases new housing construction by more than in the case of flexible wages. As a result, total output, the sum of the value added of the two sectors, increases beyond the the level of output that would be realized if prices and wages were flexible. Yet, with the two-sector production structure, a positive output gap does not necessarily lead to an increase in CPI inflation. Though positive spillover effects from the residential to the non-residential sector put upward pressure on CPI inflation, the positive technology shock also lowers marginal cost of intermediate good. The latter effect dominates and CPI inflation declines.

\section{Housing demand shocks driving a housing boom}

The models with a housing sector include new types of shocks emanating from this sector that have potentially major macroeconomic consequences. In the following, we consider a housing demand shock. It could also be called a housing preference shock, since it is modeled as random disturbance to utility from housing services. For comparison, the size of the shock is adjusted across the models such that it increases the real house prices on impact by one percent. Yet, 
Figure 21: IMPUlSE RESPONSES TO A POSITIVE TECHNOLOGY SHOCK UNDER SW RULE: MACRO VARIABLES
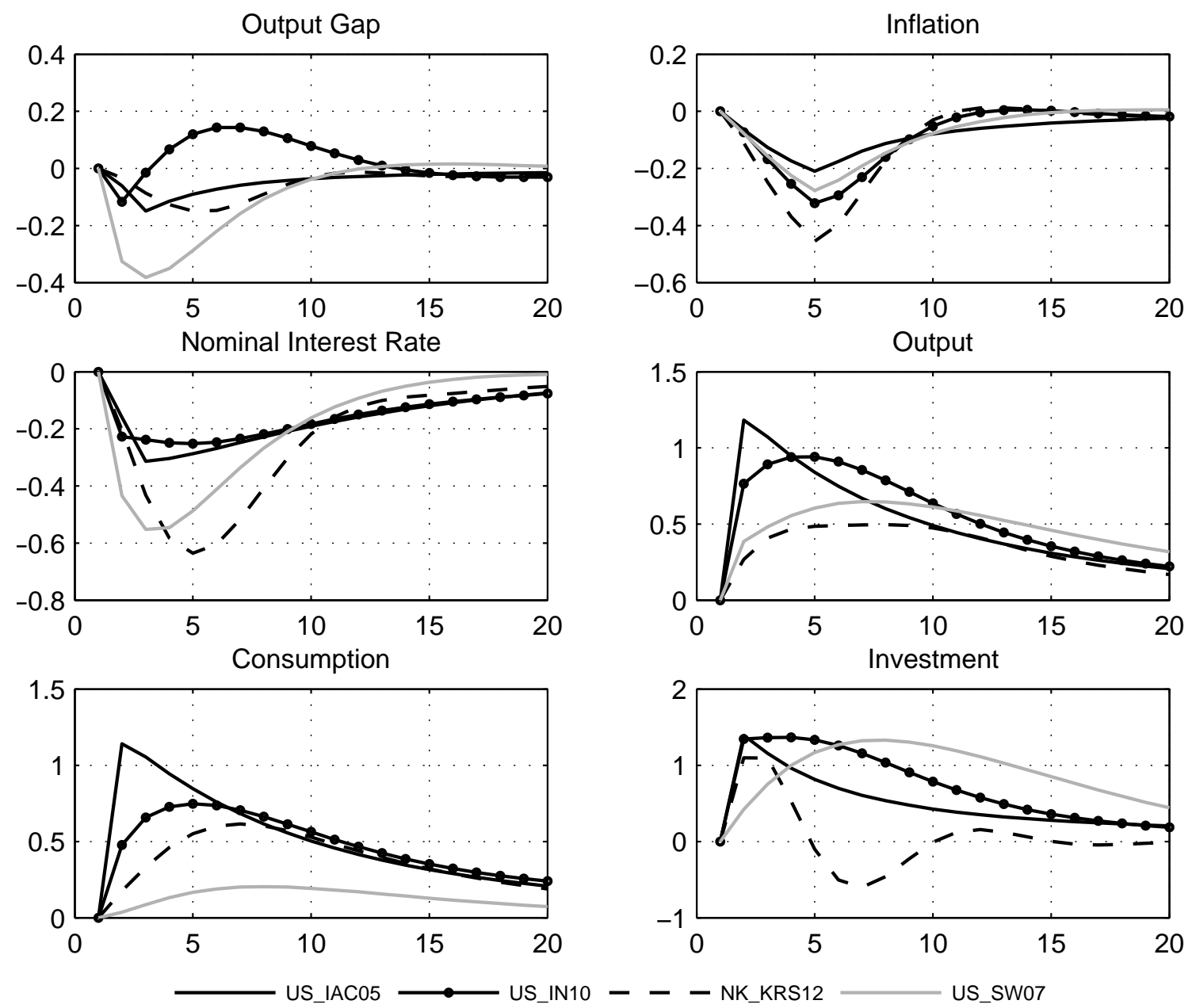

Notes: Horizontal axis represents quarters after the shock. Units of the vertical axis are percentage deviations from steady-state values. Inflation is the rate of inflation over the previous four quarters. Nominal interest rate is annualized. Other variables are expressed in quarterly terms.

we ask a slightly different question than previously with the technology shock, namely, what the consequences of such a housing demand shock would be when the degree of exogenous persistence remains model-specific 22 Under this scenario, GDP increases in all the models. The housing boom leads to an economic boom.

However, the responses of other macroeconomic and financial variables are quite different across the models as shown in Figures 23 and 24. The heterogenous dynamics reflect the different model structure and assumptions with regard to the housing market.

Unlike the US_IAC05 and US_IN10 models, house prices are subject to Calvo-type nominal frictions in the NK_KRS12 model. Thus, house prices continue to rise over one year after the

\footnotetext{
${ }^{22}$ The AR(1) coefficients of a housing demand shock for each model are following: 0.85 (US_IAC05), 0.96 (US_IN10), 0.95 (NK_KRS12)
} 
Figure 22: IMPULSE RESPONSES TO A POSITIVE TECHNOLOGY SHOCK UNDER SW RULE: INVESTMENT \& FINANCIAL VARIABLES

Nonresidential Investment
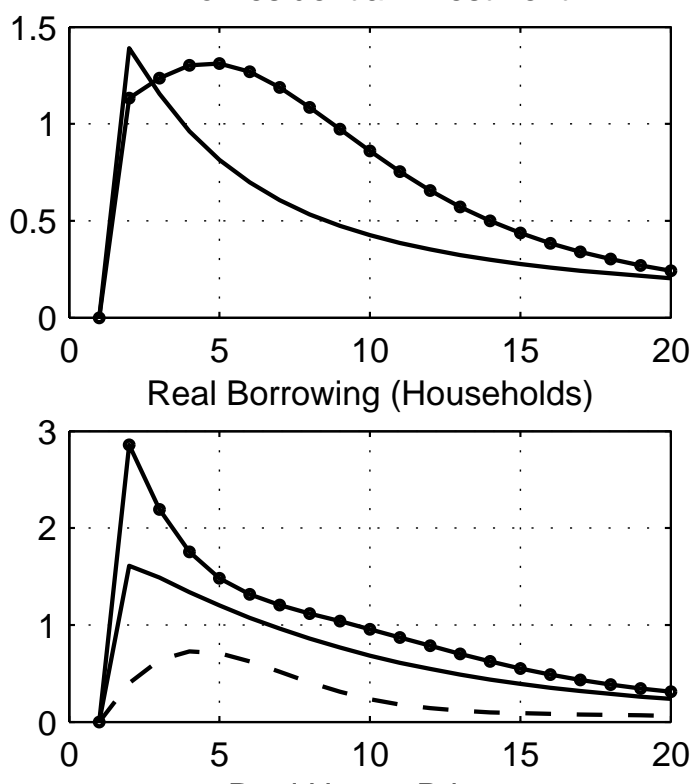

Real House Prices

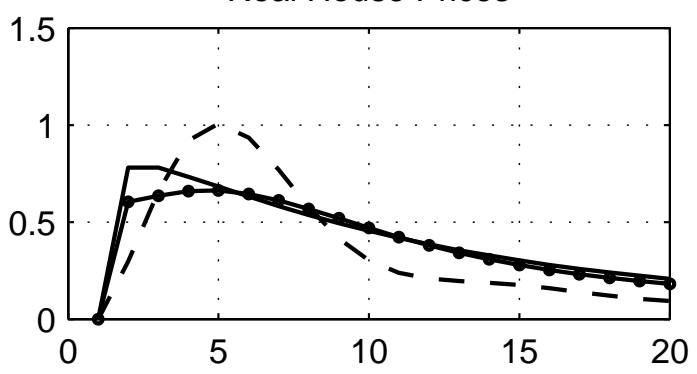

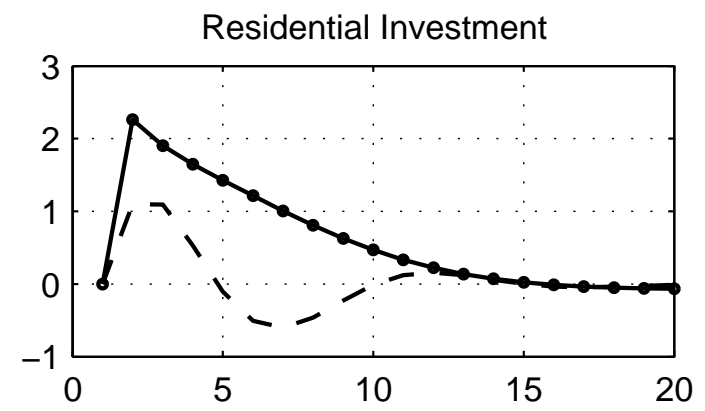

Real Borrowing (Entrepreneurs)
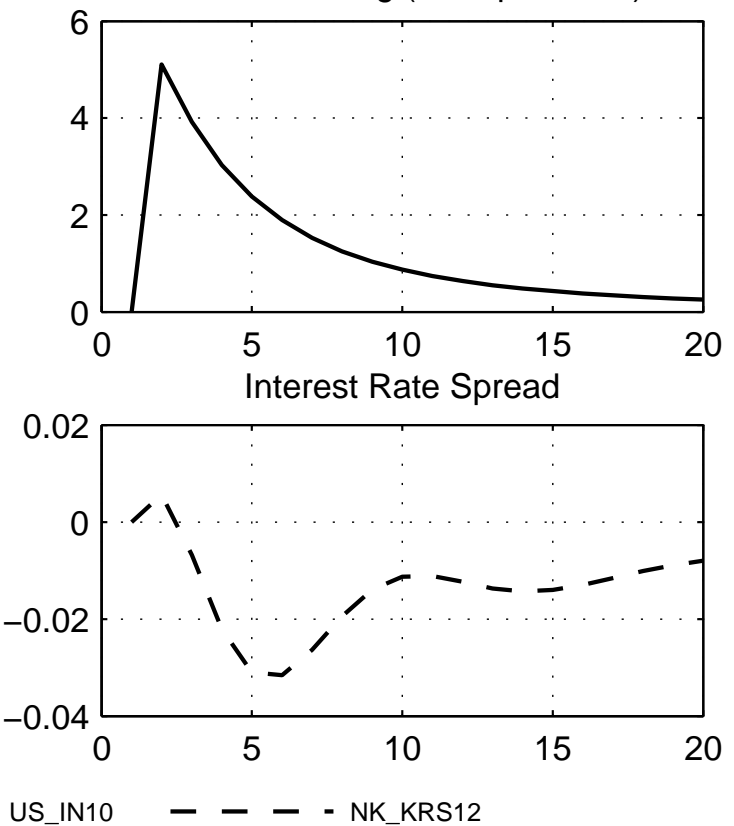

Notes: Horizontal axis represents quarters after the shock. Units of the vertical axis are percentage deviations from steady-state values. Interest rate spread is annualized. Other variables are expressed in quarterly terms.

initial shock ${ }^{23}$ The effects of such increased house prices on investment and GDP are amplified by the financial accelerator mechanism. As shown in Figure 24 the surge in residential investment and housing prices dominates the increase in households' borrowing. As households' leverage decreases, financial intermediaries charge a lower spread of the lending rate over the deposit rate. The reduced spread results in a further increase of borrowers' housing demand, which in turn leads to another increase of house prices. Actual GDP rises more than it would under flexible prices, hence a gap opens up and inflation goes up.

In the US_IAC05 model, the housing demand shock sharply pushes up consumption, investment and GDP. The increase in households' demand for housing drives up house prices. As a consequence, the collateral value of borrowers rises and the borrowing capacity is expanded. It

\footnotetext{
${ }^{23}$ The Calvo pricing parameter in housing sector is 0.75 .
} 
Figure 23: IMPULSE RESPONSES TO A POSITIVE HOUSING DEMAND SHOCK UNDER SW RULE: MACRO VARIABLES
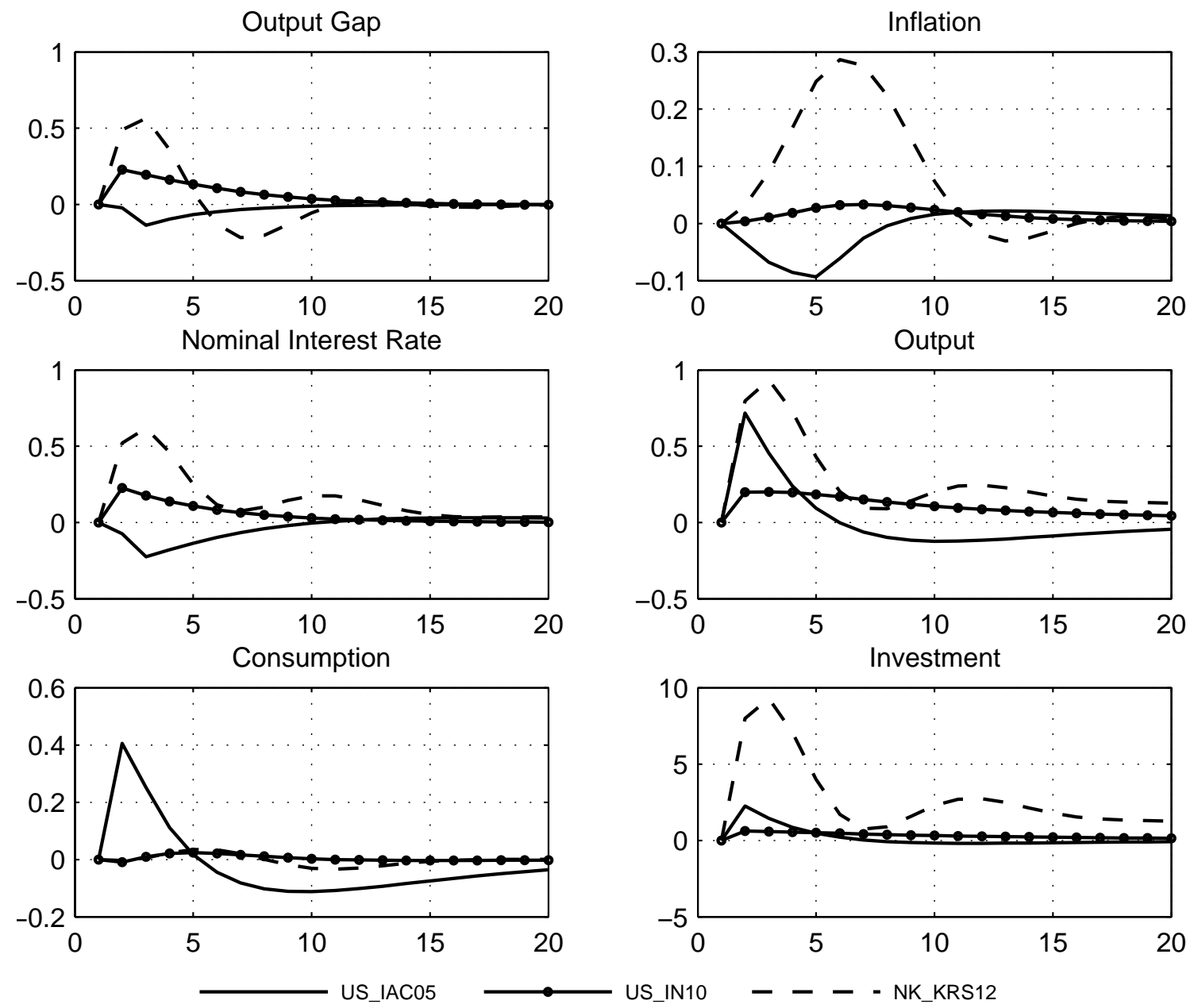

Notes: Horizontal axis represents quarters after the shock. Units of the vertical axis are percentage deviations from steady-state values. Inflation is the rate of inflation over the previous four quarters. Nominal interest rate is annualized. Other variables are expressed in quarterly terms.

leads impatient households to increase consumption and entrepreneurs to invest more. The model does not exhibit hump-shaped dynamics since it assumes no habit formation in consumption and only a small adjustment cost in nonresidential investment. Contrary to the two other models, flexible price output rises more than actual output. Accordingly, inflation declines. The reason is that the increased physical capital and housing stock raise factor productivities, thereby shifting the aggregate supply curve outwards. With borrowing-constrained entrepreneurs, the housing preference shock acts like an aggregate supply shock, which causes output and inflation to move in opposite directions.

The response of output is smallest in the US_IN10 model. The housing demand shock expands the borrowing capacity of impatient households, so that they increase consumption and housing 
Figure 24: IMPULSE RESPONSES TO A POSITIVE HOUSING DEMAND SHOCK UNDER SW RULE: INVESTMENT \& FINANCIAL VARIABLES
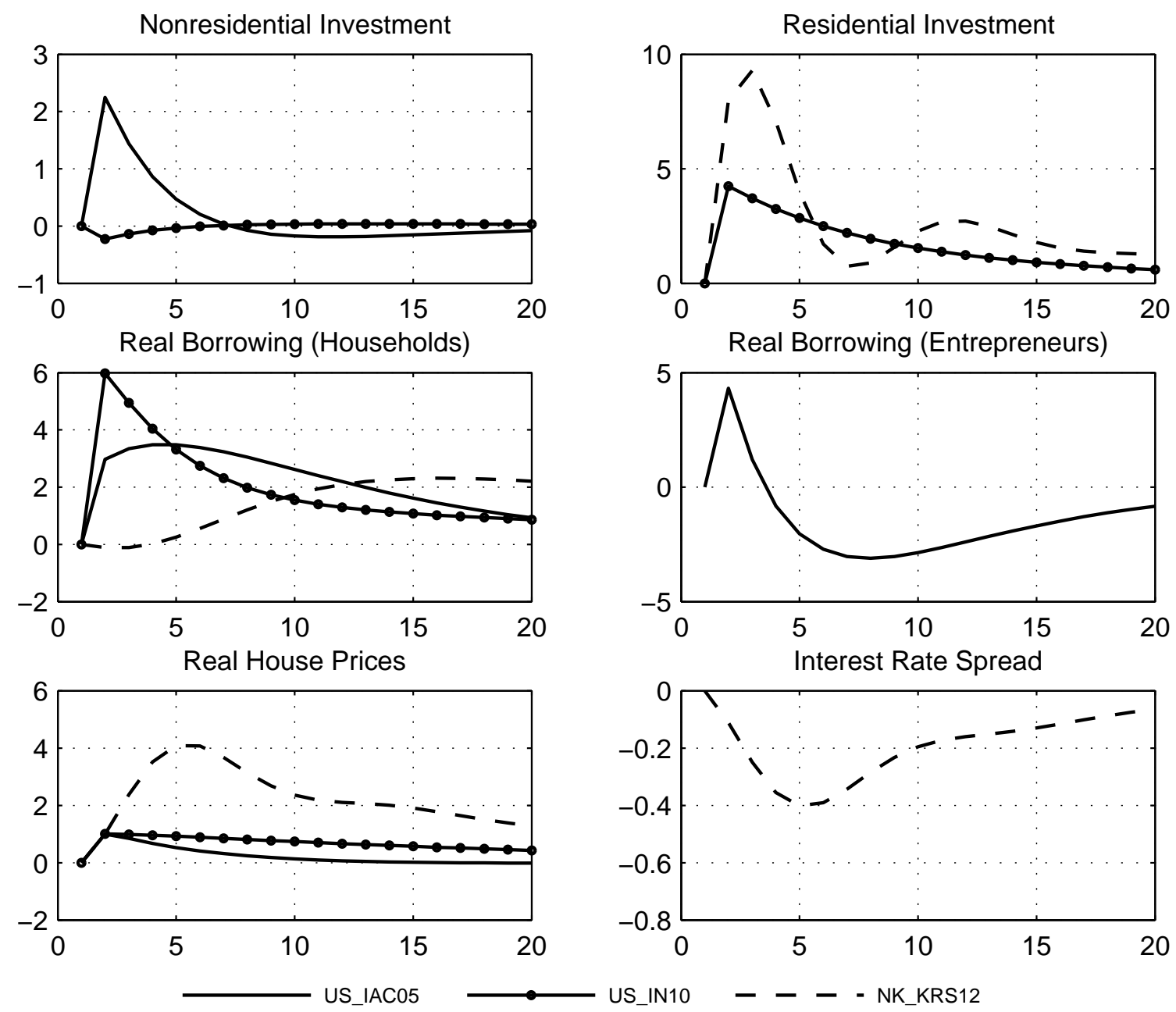

Notes: Horizontal axis represents quarters after the shock. Units of the vertical axis are percentage deviations from steady-state values. Interest rate spread is annualized. Other variables are expressed in quarterly terms.

investment. The role of the collateral channel is illustrated by the responses of residential investment and real borrowing of the households. However, patient households decrease consumption and investment in response to the increase in interest rates. Overall, GDP increases less than in the other two models.

\subsection{Propagation mechanisms: Financial intermediation and bank capital}

Finally, we explore macroeconomic consequences of shocks emanating from the banking sector. To this end, we make use of the three macro-financial models with a detailed representation of the banking sector: the model of Gertler and Karadi (2011) (NK_GK11), the model of Meh and Moran (2010) (NK_MM10) and the model of Gerali et al. (2010) (EA_GNSS10). Specifically, we 
evaluate the impact of an unanticipated reduction in bank capital on macroeconomic and financial variables. This shock can be interpreted as a sudden reduction in bank capital due to bank loan losses and asset writedowns.

The definition of the shock differs across the three models. In the NK_GK11 model the shock is modeled as a one-time wealth transfer from banks to households, whereas in the NK_MM10 model the shock is defined as sudden accelerated depreciation of bank net worth. In the EA_GNSS10 model the shock implies an unexpected deadweight loss to bank net worth. In the NK_MM10 and EA_GNSS10 models, the shock follows a first-order autoregressive process, whereas in NK_GK11 the shock is assumed to have no persistence at all 24 The size of the shock is normalized such that bank capital declines by $5 \%$ on impact in all models.

The question to be answered with this comparison exercise differs from the previous comparative analysis. Rather than investigating the consequences of bank capital shocks under a common monetary policy and a common shock process, we ask what consequences would be predicted by the different models. Thus, the scenario assumes model-specific policy rules and model-specific bank capital shock processes.

Figure 25 displays simulation outcomes of a shock that reduces bank net worth ${ }^{25}$ In all models, the decrease in bank net worth in the presence of a constraint on bank leverage leads to a decline in lending, which in turn reduces investment and output. However, in the NK_GK11 model, investment and output recover relatively quickly from the decrease, whereas in the EA_GNSS10 and NK_MM10 models, they decline for some time.

The transmission and propagation channels differ across models. In NK_GK11, the financial accelerator mechanism applies to the bank. Since bank net worth declines, financing conditions get tighter. Correspondingly, bank lending goes down, external finance premia rise sharply, and aggregate investment declines. As to the speed of return to steady-state conditions, the main reason for a faster rebound of investment is the absence of serial correlation in the bank capital shock. Household consumption increases somewhat following the one-time redistribution from the bank but declines steadily afterwards.

In EA_GNSS10, banks reduce credit supply and increase the lending rates in order to repair their balance sheets after a shortfall in bank net worth. It also depresses demand for loans via the collateral channel. As a result, investment declines. Since bank interest rates adjust only in a sticky fashion, tight financing conditions persist for several periods, depressing investment further. The decline in bank net worth is persistent. This is due to the endogenous decline of bank retained earnings as well as the exogenous persistence of the shock process. Meanwhile, household consumption slightly increases mainly due to higher wages.

In NK_MM10, the financial contract imposes a solvency condition on banks that determines banks' ability to attract funds for lending. Therefore, in response to an unanticipated fall in bank

\footnotetext{
${ }^{24}$ The autocorrelation coefficients are as follows: 0.95 (EA_GNSS10), 0.9 (NK_MM10), and 0 (NK_GK11). Gerali et al. (2010) set the parameter to 0.95 for this simulation exercise, although the median of the posterior distribution for this parameter is 0.81 .

${ }^{25}$ To perform these simulations, we use replication files for each model.
} 

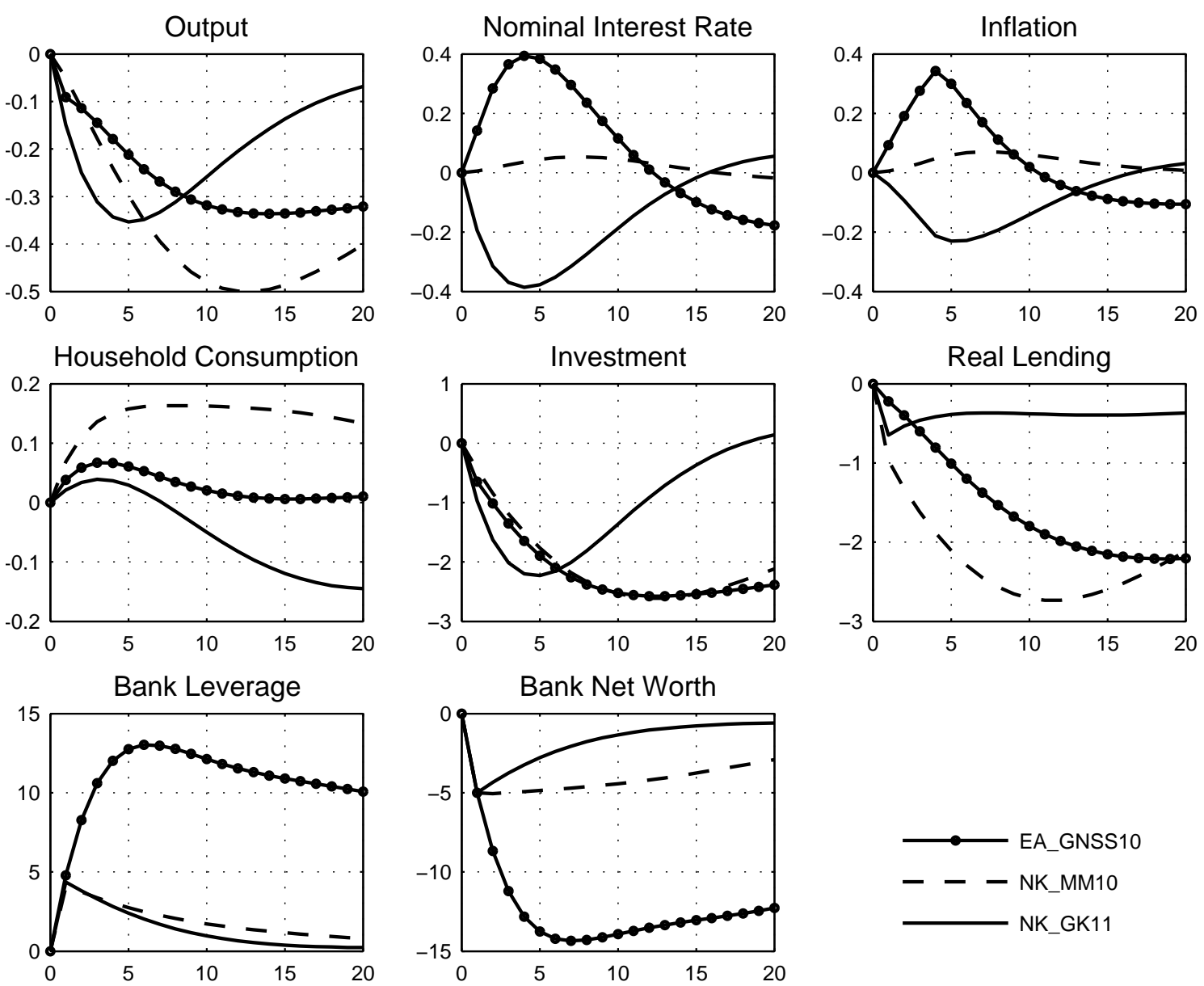

Notes: Horizontal axis represents quarters after the shock. Units of the vertical axis are percentage deviations from steady-state values. Inflation is the rate of inflation over the previous four quarters. Nominal interest rate is annualized. Other variables are expressed in quarterly terms.

net worth, banks' ability to attract funds deteriorates and they reduce lending. The decline in loan supply depresses investment, which lowers the retained earnings of banks and therefore bank net worth, reinforcing the initial shock endogenously. However, household consumption increases. The reason is that capital prices (not shown) increase in response to the shock, which in turn leads households to consume more as consumption goods become cheaper relative to capital goods.

With regard to output and inflation, the bank net worth shock appears to act as a negative demand shock in NK_GK11, where output and inflation decrease and call for monetary easing. By contrast, the shock acts as a negative supply shock in NK_MM10 and EA_GNSS10, where contraction in output is accompanied by modest inflationary pressures calling for some monetary tightening. 


\section{How to assess policy robustness: An illustrative example}

Finally, we close the series of comparative exercises with an example of how one can evaluate the robustness of policy rules under model uncertainty. The idea is simple: A policy rule is more robust than another one, if it performs better, on average, across a range of models. The search for robustness has been a central objective for many contributions to the literature on model comparison (see Section 2). Here, we just illustrate how the MMB software can be employed to this end.

The global financial crisis has been preceded by a massive credit-driven real-estate boom in the United States and other economies. If central banks had responded earlier by raising interest rates, they might have been able to avoid excessive credit growth and housing price inflation. The Taylor rule, for example, would have recommended higher interest rates ahead of the crisis. The models with financial frictions include mechanisms that can explain such credit driven booms. Thus, it is of interest to evaluate what rules perform better and whether it might be advantageous to lean-against-the wind, that is, to include an explicit reaction to credit growth into the policy rule.

\section{Participating models and rules}

In this exercise, policy performance is evaluated across four different models with financial frictions that have been estimated for the U.S. economy and have appeared in the preceding sections: the US_DG08, US_CMR14, US_IAC05 and US_IN10 models. We consider eight simple monetary policy rules (see Table 11. These include the four model-specific rules that were estimated along with the respective macro-financial model, that is, the DG08, CMR14, IAC05 and IN10 rules. They will be compared to four other simple rules: the well-known Taylor rule (see Taylor (1993a)); the SW rule; a forecast-based rule that was estimated to fit FOMC decisions in response to FOMC forecasts with real-time data by Orphanides and Wieland (2008) (OW08 rule) and a simple difference rule, that performed very well in the studies of policy robustness by Levin et al. (2003) and Orphanides and Wieland (2013). The latter is referred to as the DIF rule.

\section{Stabilization performance and robustness}

A simple central bank loss function serves as a measure of performance. It is the sum of the unconditional variance of inflation deviations from the central bank's target and the unconditional variance of the output gap. Both variances are standard simulation output in MMB.

$$
L=\operatorname{Var}\left(\pi_{t}^{z}\right)+\operatorname{Var}\left(q_{t}^{z}\right)
$$

The resulting losses are reported in Table 12 . The first row shows the losses under the modelspecific rules in the respective models. The second row indicates the performance of one of them, the CMR14 rule, in all four models. Performance deteriorates in US_DG08 and US_IAC05 while it improves in US_IN10 relative to the model-specific estimated rule. The Taylor rule delivers much more stable outcomes than any of the rules estimated with the four macro-financial models. 
Table 11: EIGHT INTEREST RATE RULES

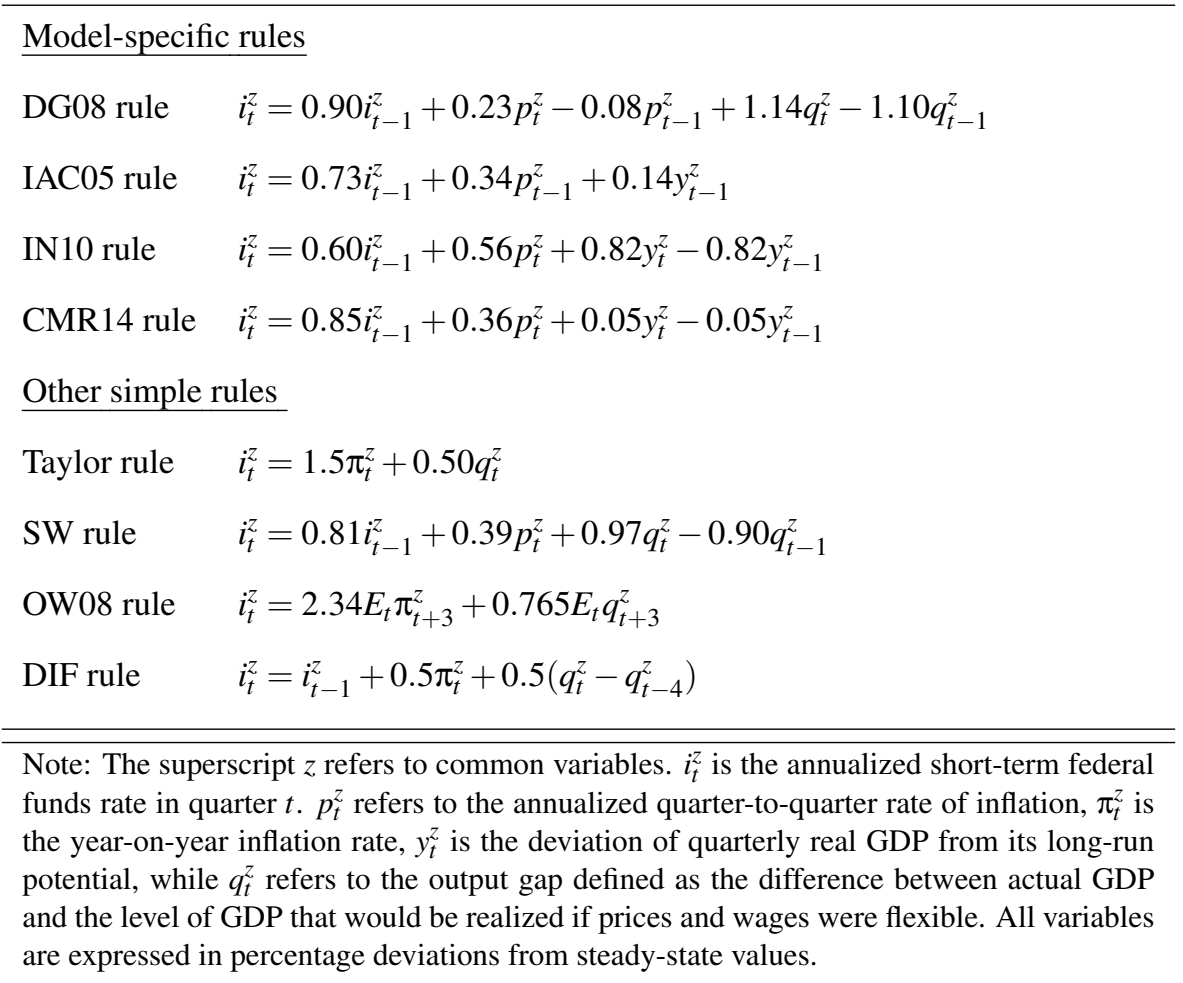

Average loss is much lower than under the CMR14 rule. The SW rule performs a little worse than Taylor's rule in US_DG08 but improves outcomes in the other three models even further.

The rule estimated on FOMC forecasts lacks robustness (OW08 rule). While it further improves performance in the US_DG08 model and US_IN10 model, losses in US_CMR14 deteriorate and it causes instability and multiple equilibria in US_IAC05. Finally, the DIF rule performs best in each of the four models and thus, also on average.

Table 12: Stabilization Performance and Robustness

\begin{tabular}{lcccc|c}
\hline & US_DG08 & US_CMR14 & US_IAC05 & US_IN10 & Average loss \\
\hline Model-specific rule & 5.8 & 47.6 & 12.3 & 6.9 & - \\
CMR14 rule & 9.1 & 47.6 & 20.4 & 3.0 & 20.0 \\
Taylor rule & 5.3 & 34.5 & 6.2 & 4.3 & 12.5 \\
SW rule & 5.7 & 19.6 & 5.1 & 3.3 & 8.3 \\
OW08 rule & 4.6 & 29.3 & $\infty$ & 3.0 & $\infty$ \\
DIF rule & 2.7 & 5.5 & 3.3 & 2.6 & 3.6 \\
\hline
\end{tabular}

Notes: The loss function is the sum of the unconditional variances of inflation and output gap. $\infty$ indicates indeterminacy. 


\section{Leaning against credit growth}

Next, we investigate whether it helps to add an explicit reaction to credit growth to the rules. The reaction coefficient on the quarterly growth rate of credit in real terms ${ }^{26}$ takes on one of two values: 0.1 and 0.3 .

Table 13 reports on the effectiveness of such a leaning-against-the-wind policy. Indeed, it helps adding a direct reaction to credit growth to the model-specific estimated rules in US_DG08, US_CMR14, US_IAC05. With a reaction coefficient of 0.1 they outperform the original modelspecific rules. In US_IN10, however, leaning against credit growth does no improve the stabilization performance of the baseline rule. In US_CMR14 and US_IAC05 a stronger reaction to credit growth (0.3) further reduces loss, whereas in US_DG08 and US_IN10 the loss increases again.

For the SW rule, at least some leaning against credit growth is beneficial in the US_DG08, US_CMR14 and US_IN10 models, but not in US_IAC05. In case of the DIF rule, which is already very effective in stabilizing output and inflation in any of the four models, leaning against credit growth is de-stabilizing, except with the US_CMR14 models.

These results suggest that some degree of leaning against credit growth can help reduce output and inflation variability. Yet, the possibility of improvement depends on the baseline rule and the particular model. If the baseline rule without credit growth is already fairly robust, leaningagainst-the-wind is more likely to hurt performance.

Table 13: POLICY RULES WITH LEANING-AGAINST-THE-WIND (CREDIT GROWTH)

\begin{tabular}{lcccc}
\hline & US_DG08 & US_CMR14 & US_IAC05 & US_IN10 \\
\hline Model-specific rule & & & & \\
Baseline & 5.8 & 47.6 & 12.3 & 6.9 \\
Leaning (0.1) & 5.3 & 28.8 & 11.4 & 7.0 \\
Leaning (0.3) & 6.1 & 19.8 & 11.3 & 7.8 \\
\hline SW rule & & & & \\
Baseline & 5.7 & 19.4 & 5.1 & 3.3 \\
Leaning (0.1) & 4.9 & 13.1 & 5.3 & 3.1 \\
Leaning (0.3) & 4.7 & 8.4 & 6.7 & 3.7 \\
\hline DIF rule & & & & \\
Baseline & 2.7 & 5.5 & 3.3 & 2.6 \\
Leaning (0.1) & 2.8 & 4.7 & 3.9 & 2.7 \\
Leaning (0.3) & 3.5 & 5.2 & 5.0 & 3.3 \\
\hline
\end{tabular}

Notes: the loss function includes the variance of inflation and the variance of the output gap.

As a next step, it would be of great interest to employ techniques for model-averaging and worst-case analysis to search for robust rules within a larger set of macro-financial models. Such

\footnotetext{
${ }^{26}$ As the model-specific rule in US_IAC05 reacts to lagged outcomes, a lagged credit growth rate is considered for this rule. In other cases, contemporaneous credit growth is used.
} 
a search could make use of optimization procedures from earlier work on policy robustness under model uncertainty (see Section 2.

\section{Critical assessment and outlook}

While there is a limited set of macroeconomic time series and country experiences, there is a multitude of macroeconomic models, and its number is growing rapidly. This is as much due to economists' creativity as to the great challenges faced by policy makers, for which they need advice and guidance based on more adequate models. There are many urgent policy questions. For example, economists need to obtain a better understanding of the macroeconomic consequences and interactions of banking regulation, private and public debt, fiscal consolidation, macroprudential policies and structural reforms. Furthermore, globalization and growth have created a demand for economic modelling expertise in many countries around the world. While academic research in macroeconomics largely focuses on the United States, Europe and Japan, central banks and government institutions in many other countries need models that are more appropriate for analyzing their economies.

At the same time, much effort is invested in building models that will never be used by anyone else. Rather than building directly on work by others, researchers usually start from scratch. Practices that would ensure easy reproducibility are not wide-spread. There is little systematic comparison of existing models.

This need not be the case. There has been tremendous progress with regard to model design, model solution techniques, econometric estimation procedures and software solutions. Many researchers are using those same techniques. While model comparison was extremely cumbersome in the past, a task reserved for meetings of teams of modelers from policy institutions, it can now be accomplished fairly easily by individual researchers. As this chapter aimed to show, comparative model analysis helps critically assessing available models, identifying similarities and differences as well as empirical inconsistencies that require more research.

The potential for comparative work has barely been tapped. Going forward, key areas for more methodological work that could rapidly bear fruit are the following: comparisons of the role of expectations formation, learning and heterogeneity; model validation and real-time estimation of competing macroeconomic models; combining statistical now-casting techniques with model-based forecasting for the medium-term; implementation of nonlinear solution techniques for occasionally-binding constraints.

Another important aspect concerns openness to competing modeling paradigms. Despite many critical assessments of the DSGE approach and its microeconomic foundations in the aftermath of the global financial crisis, DSGE modelling remains by far the most productive branch of macroeconomic modelling at this time. It takes on board elements from behavioral economics and other fields. Model comparison techniques help create standards that make it possible to compare models based on different paradigms. Thus, they support a more pluralistic yet rigorous approach to 
research in macroeconomics. 


\section{References}

Adjemian, S., Bastani, H., Juillard, M., Karame, F., Mihoubi, F., Perendia, G., Pfeifer, J., Ratto, M., Villemot, S., 2011. Dynare: Reference manual, version 4. Working Papers 1, Dynare.

Adolfson, M., Laseen, S., Linde, J., Villani, M., 2007. Bayesian estimation of an open economy DSGE model with incomplete pass-through. Journal of International Economics 72, 481-511.

Altig, D. E., Christiano, L. J., Eichenbaum, M., Linde, J., 2005. Firm-specific capital, nominal rigidities and the business cycle. Discussion Paper 4858, CEPR.

Anderson, G. S., Moore, G., 1985. A linear algebraic procedure for solving linear perfect foresight models. Economics Letters 17 (3), 247-252.

Ball, L., 1999. Policy rules for open economies. In: Taylor, J. B. (Ed.), Monetary Policy Rules. Chicago: University of Chicago Press.

Bernanke, B., Gertler, M., Gilchrist, S., 1999. The financial accelerator in a quantitative business cycles framework. In: Taylor, J. B., Woodford, M. (Eds.), Handbook of Macroeconomics Volume 1C. Amsterdam: Elsevier Science, North-Holland.

Bryant, R., Currie, D., Frenkel, J., Masson, P., Portes, R. (Eds.), 1989. Macroeconomic Policies in an Interdependent World. Washington, D.C.: The Brookings Institution.

Bryant, R., Henderson, D. W., Holtham, G., Hooper, P., Symansky, S. A. (Eds.), 1988. Empirical Macroeconomics for Interdependent Economies. Washington, D.C.: The Brookings Institution.

Bryant, R., Hooper, P., Mann, C. (Eds.), 1993. Evaluating Policy Regimes: New Research in Empirical Macroeconomics. Washington, D.C.: The Brookings Institution.

Carabenciov, I., Ermolaev, I., Freedman, C., Juillard, M., Kamenik, O., Korshunov, D., Laxton, D., 2008. A small quarterly projection model of the US economy. Tech. Rep. 08/278, IMF.

Christensen, I., Dib, A., 2008. The financial accelerator in an estimated New Keynesian model. Review of Economic Dynamics 11, 155-178.

Christiano, L., Eichenbaum, M., Rebelo, S., 2011. When Is the Government Spending Multiplier Large? Journal of Political Economy 119 (1), 78 - 121.

Christiano, L. J., 2002. Solving dynamic equilibrium models by a method of undetermined coefficients. Computational Economics 20(1-2), 21-55.

Christiano, L. J., Eichenbaum, M., Evans, C. L., 2005. Nominal rigidities and the dynamic effects of a shock to monetary policy. Journal of Political Economy 113(1), 1-45.

Christiano, L. J., Motto, R., Rostagno, M., 2014. Risk shocks. American Economic Review $104(1), 27-65$. 
Christoffel, K., Kuester, K., Linzert, T., 2009. The role of labor markets for euro area monetary policy. European Economic Review 53, 908-936.

Claerbout, J., 1994. Hypertext documents about reproducible research. URL http://sepwww.stanford.edu/sep/jon/nrc.html

Coenen, G., Erceg, C. J., Freedman, C., Furceri, D., Kumhof, M., Lalonde, R., Laxton, D., Linde, J., Mourougane, A., Muir, D., Mursula, S., de Resende, C., Roberts, J., Roeger, W., Snudden, S., Trabandt, M., in't Veld, J., 2012. Effects of fiscal stimulus in structural models. American Economic Journal: Macroeconomics 4, 22-68.

Cogan, J., Cwik, T., Taylor, J., Wieland, V., 2010. New keynesian versus old keynesian government spending multipliers. Journal of Economic Dynamics and Control 34, 281-295.

Cogan, J., Taylor, J., Wieland, V., Wolters, M., 2013. Fiscal consolidation strategy. Journal of Economic Dynamics and Control 37, 404-421.

Collard, F., Juillard, M., 2001. Accuracy of stochastic perturbation methods: The case of asset pricing models. Journal of Economic Dynamics \& Control 25, 979-999.

Cwik, T., Wieland, V., July 2011. Keynesian government spending multipliers and spillovers in the euro area. Economic Policy 26, 493-549.

De Graeve, F., 2008. The external finance premium and the macroeconomy: US post-WWII evidence. Journal of Economic Dynamics and Control 32, 3415-3440.

Donoho, D., 2010. An invitation to reproducible computational research. Biostatistics 11(3), 385388.

Drautzburg, T., Uhlig, H., 2015. Fiscal stimulus and discretionary taxation. Review of Economic Dynamics 18 (4), 894-920.

Fair, R. C., Taylor, J. B., 1983. Solution and maximum likelihood estimation of dynamic nonlinear rational expectations models. Econometrica 51, 1169-1185.

Fomel, S., Claerbout, J. F., 2009. Reproducible research. Computing in Science and Engineering 2009(1), 5-7.

Frankel, J., Rockett, K., 1988. International macroeconomic policy coordination when policymakers do not agree on the true model. The American Economic Review 78 (3), 318-340.

Freire, J., Bonnet, P., Shasha, D., 2012. Computational reproducibility: State-of-the-art, challenges, and database research opportunities. Proceedings of SIGMOD, 593-596.

Fuhrer, J. C., 1997. Inflation/output variance trade-offs and optimal monetary policy. Journal of Money, Credit and Banking 29(2), 214-234.

Gelain, P., 2010. The external finance premium in the euro area: A dynamic stochastic general 
equilibrium analysis. North American Journal of Economics and Finance 21, 49-71.

Gerali, A., Neri, S., Sessa, L., Signoretti, F. M., 2010. Credit and banking in a dsge model of the euro area. Journal of Money, Credit and Banking 42 (s1), 107-141.

Gerke, R., Jonsson, M., Kliem, M., Kolasa, M., Lafourcade, P., Locarno, A., Makarski, K., McAdam, P., 2013. Assessing macro-financial linkages: A model comparison exercise. Economic Modelling 31 (C), 253-264.

Gertler, M., Karadi, P., January 2011. A model of unconventional monetary policy. Journal of Monetary Economics 58 (1), 17-34.

Guerrieri, L., Iacoviello, M., Covas, F. B., Driscoll, J. C., Kiley, M. T., Jahan-Parwvar, M., Olive, A. Q., Sim, J. W., 2015. Macroeconomic effects of banking sector losses across structural models. Finance and Economics Discussion Series 2015-044, Board of Governors of the Federal Reserve System.

Hughes-Hallett, A., 1989. Empirical macroeconomics for interdependent economies: Book review. Journal of International Economics 26 (1 - 2), 189 - 194.

Hughes-Hallett, A., Wallis, K. F. (Eds.), 2004. EMU Macroeconomic Model Comparison excercise for the Euroconference 7-8 June 2002. Economic Modelling 21(5).

Iacoviello, M., 2005. House prices, borrowing constraints, and monetary policy in the business cycle. The American Economic Review 95(3), 739-764.

Iacoviello, M., Neri, S., April 2010. Housing market spillovers: Evidence from an estimated dsge model. American Economic Journal: Macroeconomics 2 (2), 125-64.

Ireland, P., 2003. Endogenous money or sticky prices? Journal of Monetary Economics 50, 16231648 .

Ireland, P., 2011. A New Keynesian perspective on the Great Recession. Journal of Money, Credit and Banking 43(1), 31-54.

Judd, K. (Ed.), 1998. Numerical Methods in Economics. MIT Press.

Juillard, M., 2001. Dynare: A program for the simulation of rational expectation models, computing in Economics and Finance 213.

Kannan, P., Rabanal, P., Scott, A. M., 2012. Monetary and macroprudential policy rules in a model with house price booms. The B.E. Journal of Macroeconomics 12 (1), 16.

Kilponen, J., Pisani, M., Schmidt, S., Corbo, V., Hledik, T., Hollmayr, J., Hurtado, S., Julio, P., Lozej, M., Landfall, H., Maria, J. R., Micallef, B., Papageorgiou, D., Rysanek, J., Sideris, D., Thomas, C., De Walque, G., 2015. Comparing fiscal multipliers across models and countries in europe. Working Paper Series 1760, European Central Bank. 
Kiyotaki, N., Moore, J., 1997. Credit cycles. Journal of Political Economy 105(2), 211-48.

Klein, L. (Ed.), 1991. Comparative Performance of U.S. Econometric Models. Oxford, Eng.: Oxford University Press.

Klein, P., 2000. Using the generalized Schur form to solve a multivariate linear rational expectations model. Journal of Economic Dynamics and Control 24(10), 1405-1423.

Kuester, K., Wieland, V., 2010. Insurance policies for monetary policy in the euro area. Journal of the European Economic Association 8(4), 872-912.

Levin, A., Wieland, V., Williams, J. C., 1999. Robustness of simple monetary policy rules under model uncertainty. In: Taylor, J. B. (Ed.), Monetary Policy Rules. Chicago: University of Chicago Press.

Levin, A., Wieland, V., Williams, J. C., 2003. The performance of forecast-based monetary policy rules under model uncertainty. The American Economic Review 93(3), 622-645.

Mankiw, N. G., Reis, R., 2007. Sticky information in general equilibrium. Journal of the European Economic Association 5(2-3), 603-613.

McCallum, B., Nelson, E., 1999. Performance of operational policy rules in an estimated semiclassical structural model. In: Taylor, J. B. (Ed.), Monetary Policy Rules. Chicago: University of Chicago Press.

Meh, C. A., Moran, K., March 2010. The role of bank capital in the propagation of shocks. Journal of Economic Dynamics and Control 34 (3), 555-576.

Mortensen, D., Pissarides, C., 1994. Job creation and job desctruction in the theory of unemployment. Review of Economic Studies 61(3), 397-415.

Orphanides, A., 2003. The quest for prosperity without inflation. Journal of Monetary Economics $50,633-663$.

Orphanides, A., Wieland, V., 2008. Economic projections and rules of thumb for monetary policy. Fed of St. Louis Review, 307-324.

Orphanides, A., Wieland, V., 2013. Complexity and monetary policy. Journal of International Central Banking 9(1), 167-204.

Quint, D., Rabanal, P., June 2014. Monetary and Macroprudential Policy in an Estimated DSGE Model of the Euro Area. International Journal of Central Banking, International Journal of Central Banking 10 (2), 169-236.

Rabanal, P., 2007. Does inflation increase after a monetary policy tightening? answers based on a estimated DSGE model. Journal of Economic Dynamics \& Control 31, 906-937.

Rabanal, P., 2009. Inflation differentials between Spain and the EMU: A DSGE perspective. Jour- 
nal of Money, Credit and Banking 41(6), 1141-1166.

Ratto, M., Roeger, W., in 't Veld, J., 2009. QUEST III: An estimated open-economy DSGE model of the euro area with fiscal and monetary policy. Economic Modelling 26(1), 222-233.

Romer, C., Bernstein, J., January 8 2009. The job impact of the american recovery and reinvestment plan.

Rotemberg, J. J., Woodford, M., 1997. An optimization-based econometric framework for the evaluation of monetary policy. NBER Macroeconomics Annual 12, 297-346.

Rudebusch, G. D., Svensson, L. E. O., 1999. Policy rules for inflation targeting. In: Taylor, J. B. (Ed.), Monetary Policy Rules. Chicago: University of Chicago Press.

Sandve, G. K., Nekrutenko, A., Taylor, J., Hovig, E., 2013. Ten simple rules for reproducible computational research. PLOS Computational Biology 9(10), 1-4.

Schmidt, S., Wieland, V., 2013. The new keynesian approach to dynamic general equilibrium modeling: Models, methods and macroeconomic policy evaluation. In: Dixon, P. B., Jorgenson, D. W. (Eds.), Handbook of Computable General Equilibrium Modeling. North Holland.

Sims, C., 2001. Solving linear rational expectations models. Journal of Computational Economics 20(1-2), 1-20.

Slobodyan, S., Wouters, R., 2012. Learning in an estimated medium-scale DSGE model, journal of Economic Dynamics and Control.

Smets, F., Wouters, R., 2003. An estimated dynamic stochastic general equilibrium model of the euro area. Journal of the European Economic Association 1 (5), 1123-1175.

Smets, F., Wouters, R., 2007. Shocks and frictions in US business cycles: A bayesian DSGE approach. The American Economic Review 97(3), 586-606.

Taylor, J. B., 1993a. Discretion versus policy rules in practice. Carnegie-Rochester Conference Series on Public Policy 39, 195-214.

Taylor, J. B., 1993b. Macroeconomic Policy in a World Economy. W.W. Norton, New York, online Edition available on: http://www.stanford.edu/ johntayl/MacroPolicyWorld.htm.

Taylor, J. B. (Ed.), 1999. Monetary Policy Rules. The University of Chicago Press.

Taylor, J. B., Wieland, V., 2012. Surprising comparative properties of monetary models: Results from a new data base. Review of Economics and Statistics 94(3), 800-816.

Uhlig, H., 1995. A toolkit for analyzing nonlinear dynamic stochastic models easily, discussion Paper 97, Tilburg University, Center for Economic Research.

Wallis, K. F., 2004. Comparing empirical models of the euro area economy. Economic Modelling 21(5) 21, 735-758. 
White, W. W. (Ed.), 1978. Computers and Mathematical Programming: Proceedings of the Bicentennial Conference on Mathematical Programming held at the National Bureau of Standards, Gaithersburg, Maryland, November 29 - December 1, 1976, Band 13. U.S. Government Printing Office, Washington.

Wieland, V., Cwik, T., Mueller, G. J., Schmidt, S., Wolters, M., 2012. A new comparative approach to macroeconomic modeling and policy analysis. Journal of Economic Behavior \& Organization 83 (3), 523-541.

Wieland, V., Wolters, M., 2013. Forecasting and policy making. In: Elliott, G., Timmermann, A. (Eds.), Handbook of Economic Forecasting. North Holland. 


\section{IMFS WORKING PAPER SERIES}

\section{Recent Issues}

\begin{tabular}{|c|c|c|}
\hline $106 / 2016$ & Helmut Siekmann & $\begin{array}{l}\text { Konstruktionsfehler bei der } \\
\text { Einlagensicherung auf EU-Ebene }\end{array}$ \\
\hline $105 / 2016$ & Athanasios Orphanides & $\begin{array}{l}\text { Fiscal Implications of Central Bank } \\
\text { Balance Sheet Policies }\end{array}$ \\
\hline $104 / 2016$ & Helmut Siekmann & $\begin{array}{l}\text { Preis- und Finanzstabilität: der Primat der } \\
\text { Politik, der rechtliche Rahmen und das } \\
\text { „ökonomische Gesetz" }\end{array}$ \\
\hline $103 / 2016$ & $\begin{array}{l}\text { John B. Taylor } \\
\text { Volker Wieland }\end{array}$ & $\begin{array}{l}\text { Finding the Equilibrium Real Interest Rate } \\
\text { in a Fog of Policy Deviations }\end{array}$ \\
\hline $102 / 2016$ & $\begin{array}{l}\text { Tilman Bletzinger } \\
\text { Volker Wieland }\end{array}$ & $\begin{array}{l}\text { Forward guidance and "lower for longer": } \\
\text { The case of the ECB }\end{array}$ \\
\hline $101 / 2016$ & Helmut Siekmann & $\begin{array}{l}\text { Ziele, Aufgaben und Befugnisse des } \\
\text { Europäischen Systems der Zentralbanken } \\
\text { (ESZB) }\end{array}$ \\
\hline $100 / 2016$ & $\begin{array}{l}\text { Robert C. M. Beyer } \\
\text { Volker Wieland }\end{array}$ & $\begin{array}{l}\text { Schätzung des mittelfristigen Gleich- } \\
\text { gewichtszinses in den Vereinigten Staaten, } \\
\text { Deutschland und dem Euro-Raum mit der } \\
\text { Laubach-Williams-Methode }\end{array}$ \\
\hline
\end{tabular}

99 / $2015 \quad$ Helmut Siekmann

Exit, Exclusion, and Parallel Currencies in the Euro Area

98 / 2015 Helmut Siekmann

Stellungnahme für die öffentliche Anhörung der Verfassungskommission des Landtags Nordrhein-Westfalen zur Schuldenbremse
97 I $2015 \quad$ José María Liberti Amit Seru
Vikrant Vig
96 / $2015 \quad$ Patrick Tuschl
Rechtsgrundlagen für die Vereinbarkeit von Finanzhilfen für Kreditinstitute mit dem Beihilferecht der EU im Rahmen der Finanzmarktkrise

$95 / 2015$ Athanasios Orphanides

Fear of Liftoff: Uncertainty, Rules and Discretion in Monetary Policy Normalization

94 / 2015

Bettina Brüggemann Jinhyuk Yoo

Aggregate and Distributional Effects of Increasing Taxes on Top Income Earners

93 / 2015 Tobias H. Tröger

Regulatory Influence on Market Conditions in the Banking Union 


\section{I $2015 \quad$ Janis Skrastins Vikrant Vig}

$91 / 2015$

$90 / 2015$

Florian Hoffmann

Roman Inderst

Marcus Opp

Helmut Siekmann

89 / 2015

Helmut Siekmann

$88 / 2015$

87 / 2015

$86 / 2015$

$85 / 2014$

84 / 2014

Athanasios Orphanides

$83 / 2014$

82 / 2014

$81 / 2014$

$80 / 2014$

$79 / 2014$
Tobias H. Tröger

Florian Deuflhard

Dimitris Georgarakos

Roman Inderst

Falko Fecht

Roman Inderst

Sebastian Pfeil

Markus Behn

Rainer Haselmann

Thomas Kick

Vikrant Vig

Elena Afanasyeva

Jochen Güntner

Markus Behn

Rainer Haselmann

Vikrant Vig

Volker Wieland

Maik Wolters

Hermann Remsperger

Athanasios Orphanides
How Organizational Hierarchy Affects Information Production

Regulating Deferred Incentive Pay

The Legality of Outright Monetary

Transactions (OMT) of the European

System of Central Banks

(publ. in: Rövekamp / Bälz / Hilpert (eds.),

Central Banking and Financial Stability in

East Asia, 2015, pp. 101-123)

The Legal Framework for the European

System of Central Banks

(publ. in: Rövekamp / Bälz / Hilpert (eds.),

Central Banking and Financial Stability in

East Asia, 2015, pp. 43-86)

Financial Literacy and Savings Account Returns

A Theory of the Boundaries of Banks with Implications For Financial Integration and Regulation

The Political Economy of Bank Bailouts

Lending Standards, Credit Booms and Monetary Policy

Are Rules and Boundaries Sufficient to Limit Harmful Central Bank Discretion? Lessons from Europe

How Special Are They? - Targeting Systemic Risk by Regulating Shadow Banking

The Limits of Model-Based Regulation

Is there a threat of self-reinforcing deflation in the Euro area? A view through the lens of the Phillips curve

Der makroprudenzielle Komplex: der Prozess, das Schloss, das Urteil

What Happened in Cyprus? The Economic Consequences of the Last Communist Government in Europe 